\begin{tabular}{|c|l|}
\hline Title & Stability for evolving graphs by nonlocal weighted curvature \\
\hline Author(s) & Giga, M.-H; Giga, Y. \\
\hline Citation & Hokkaido University Preprint Series in Mathematics, 372, 1-70 \\
\hline Issue Date & 1997-3-1 \\
\hline DOI & 10.14943/83518 \\
\hline Doc URL & http://hdl.handle.net/2115/69122 \\
\hline Type & bulletin (article) \\
\hline File Information & pre372.pdf \\
\hline
\end{tabular}

Instructions for use 


\section{STABILITY FOR EVOLVING \\ GRAPHS BY NONLOCAL WEIGHTED CURVATURE}

M.-H. Giga and Y. Giga

Series \#372. March 1997 


\section{HOKKAIDO UNIVERSITY \\ PREPRINT SERIES IN MATHEMATICS}

\#348 Y. Ito and I. Nakamura, Hilbert schemes and simple singularities $A_{n}$ and $D_{n}, 22$ pages. 1996

\#349 R. Agemi and K. Yokoyama, The null condition and global existence of solutions to systems of wave equations with different speeds, 42 pages. 1996

\#350 F. Hiroshima, Weak coupling limit with a removal of an ultraviolet cut-off for a Hamiltonian of particles interacting with a massive scalar field, 21 pages. 1996.

\#351 T. Nakazi and Y. Watatani, Invariant subspace theorems for subdiagonal algebras, 22 pages. 1996.

\#352 Y. Nishiura and H. Suzuki, Nonexistence of stable turing patterns with smooth limiting interfacial configurations in higher dimensional spaces, 21 pages. 1996.

\#353 Y.-G. Chen, Y. Giga and K. Sato, On instant extinction for very fast diffusion equations, 9 pages. 1996.

\#354 A. Gyoja and H. Yamashita, Associated variety, Kostant-Sekiguchi correspondence, and locally free $U(\mathrm{n})$-action on Harish-Chandra modules, 25 pages. 1996.

\#355 G. Ishikawa, Topology of plane trigonometric curves and the strangeness of plane curves derived from real pseudo-line arrangements, 18 pages. 1996.

\#356 N.H. Bingham and A. Inoue, The Drasin-Shea-Jordan theorem for Hankel transforms of arbitrarily large order, 13 pages. 1996.

\#357 S. Izumiya, Singularities of solutions for first order partial differential equations, 20 pages. 1996.

\#358 N. Hayashi, P.I. Naumkin and T. Ozawa, Scattering theory for the Hartree equation, 14 pages. 1996.

\#359 I. Tsuda and K. Tadaki, A logic-based dynamical theory for a genesis of biological threshold, 49 pages. 1996.

\#360 I. Tsuda and A. Yamaguchi, Singular-continuous nowhere-differentiable attractors in neural systems, 40 pages. 1996.

\#361 M. Nakamura and T. Ozawa, Low energy scattering for nonlinear Schröclinger equations in fractional order Sobolev spaces, 17 pages. 1996.

\#362 I. Nakamura, Hilbert schemes and simple singularities $E_{6}, E_{7}$ and $E_{8}, 21$ pages. 1996.

\#363 T. Mikami, Equivalent conditions on the central limit theorem for a sequence of probability measures on $R, 7$ pages. 1996 .

\#364 S. Izumiya and T. Sano, Generic affine differential geometry of space curves, 23 pages. 1996.

\#365 T. Tsukada, Stability of reticular optical caustics, 12 pages. 1996.

\#366 A. Arai and M. Hirokawa, On the existence and uniqueness of ground states of a generalized spin-boson model, 40 pages. 1996.

\#367 A. Arai, A class of representations of the *-algebra of the canonical commutation relations over a Hilbert space and instability of embedded eigenvalues in quantum field models, 12 pages. 1996.

\#368 K. Ito, BV-solutions of a hyperbolic-elliptic system for a radiating gas, 33 pages. 1997.

\#369 M. Nakamura and T. Ozawa, Nonlinear Schrödinger equations in the Sobolev space of critical order, 20 pages. 1997.

\#370 N.H. Bingham and A. Inoue, An Abel-Tauber theorem for Hankel transforms, 8 pages. 1997.

\#371 T. Nakazi and H. Sawada, The commutator ideal in Toeplitz algebras for uniform algebras and the analytic structure, 9 pages. 1997. 


\title{
STABILITY FOR EVOLVING GRAPHS BY NONLOCAL WEIGHTED CURVATURE
}

\author{
Dedicated to Professor Kŷिya Masuda \\ on the occasion of his 60th birthday \\ Mi-Ho Giga and Yoshikazu Giga \\ Department of Mathematics, Hokkaido University \\ Sapporo 060, Japan
}

\begin{abstract}
A general stability and convergence theorem is established for generalized solutions of a family of nonlinear evolution equations with nonlocal diffusion in one space dimension. As the first application motion by nonlocal weighted curvature is approximated by solutions of regular problem, when initial curve is given as the graph of a continuous periodic function. This justifies the motion by crystalline energy as a limit of regularized problems. As the second application the motion by crystalline energy is shown to approximate the motion by regular interfacial energy if the crystalline energy approximates the regular energy. This gives the convergence of crystalline algorithm for general curvature flow equations. Our general results are also important to explain that geometric evolution of crystals depends continuously on temperature even if facets appear.
\end{abstract}

\section{Contents.}

1. Introduction

2. Definition and main results

3. Sufficient condition for solutions

4. Wulff functions 

5. Convergence of Wulff functions
6. Proof of General stability theorem
7. Construction of barriers
Appendix

\section{§1. Introduction.}

In [GG1-3] extending the theory [CIL] of viscosity solutions, we introduced a very general notion of graph-like solutions for motion driven by nonlocal weighted curvature in the plane and established the unique global existence of solutions for general continuous (periodic) initial data. In this paper we are interested in convergence of solutions for equations approximating the original equation.

To motivate our problem we begin with a simple example of equations in one space dimension which are quasilinear. We consider

$$
u_{t}-\Lambda_{W}(u)=0 \quad \text { with } \Lambda_{W}(u)=\left(W^{\prime}\left(u_{x}\right)\right)_{x}
$$

with a given convex function $W$ on $\mathbf{R}$ whose derivative $W^{\prime}$ may have jumps. Here subscripts $t$ and $x$ denote partial differentiation in time and space variables, respectively. The equation (1.1) is a simple example of fully nonlinear equation

$$
u_{t}+F\left(u_{x}, \Lambda_{W}(u)\right)=0
$$

with continuous $F$ satisfying degenerate ellipticity:

$$
F(p, X) \leq F(p, Y) \quad \text { for } X \geq Y \text {. }
$$

If $W(p)=p^{2} / 2$, the equation (1.1) is the heat equation, of course. If $W(p)=$ $\left(1+p^{2}\right)^{1 / 2}, \Lambda_{W}(u)$ is the curvature of the graph of $u(t, \cdot)$ so $\Lambda_{W}$ is often called the weighted curvature and $W$ is called an (interfacial) energy (density). The value $\Lambda_{W}$ is actually determined by the second distributional derivative $W^{\prime \prime}$ of $W$; however, we denote it by $\Lambda_{W}$ instead of $\Lambda_{W^{\prime \prime}}$. If $W(p)=|p|$, then (1.1) formally becomes

$$
u_{t}-2 \delta\left(u_{x}\right) u_{x x}=0
$$


where $\delta$ is the Dirac delta function. The interpretation of (1.1) or even (1.3) is not clear in naive sense. We consider the initial value problem for (1.1) with initial condition

$$
u(0, x)=u_{0}(x)
$$

- where $u_{0}$ is assumed to be continuous and, for simplicity, periodic with period $\varpi$ as in [GG3], i.e. $u_{0} \in C(\mathbf{T})$ with $\mathbf{T}=\mathbf{R} / \varpi \mathbf{Z}$.

We introduced notion of generalized solution for (1.1) or even (1.2) by extending the theory of viscosity solutions. We proved that there exists a global unique continuous generalized solution of (1.2), (1.4) by constructing sub- and supersolutions [GG3]. There we have assumed that $W$ is $C^{2}$ except a discrete set and that $W^{\prime \prime}$ is bounded on every compact set excluding the points where $W$ is not $C^{2}$; we say $W \in \mathcal{E}$ if $W$ is convex and satisfies these conditions. The class $\mathcal{E}$ includes piecewise linear convex functions and other interesting examples, so the assumption $W \in \mathcal{E}$ is not restrictive for applications.

Another way to construct a solution of (1.1), (1.4) is an approximation method. We approximate $W$ by another energy $W_{\varepsilon} \in C^{\infty}(\mathbf{R})$ with $W_{\varepsilon}^{\prime \prime}>0$ and solve the approximate equation

$$
u_{t}-\Lambda_{W_{c}}(u)=0
$$

with

$$
u(0, x)=u_{0}^{\varepsilon}(x)
$$

approximating $u_{0}$ with $u_{0}^{e} \in C^{\infty}(\mathbf{T})$. By $W_{\varepsilon}^{\prime \prime}>0$ the equation (1.5) is nondegenerate parabolic. Since a solution $u^{\varepsilon}$ of $(1.5),(1.6)$ fulfills a priori estimate

$$
\left|\left(u^{e}\right)_{x}\right|(t, x) \leq \max \left|\left(u_{0}^{\varepsilon}\right)_{x}\right|, \quad x \in \mathbf{R}, t>0
$$

by the maximum principle, the classical theory of uniformly parabolic equations yields the unique global smooth solution of (1.5), (1.6) [LSU]. The problem is whether or not the limit of $u^{\varepsilon}$ converges to a function $u$ as $\varepsilon \rightarrow 0$ and $u$ solves $(1.1),(1.4)$ in the sense of [GG3]. Our main convergence results (Theorem 2.11 with Remark 2.10) apply various problems including this problem. We state a version for autonomous equations, i.e. $F_{e}$ and $F$ is time-independent in Theorem 2.11. 
Theorem 1.1 (Convergence). Let $F_{\varepsilon}, F_{0} \in C\left(\mathbf{R}^{2}\right)$ be degenerate elliptic for $\varepsilon>0$. Assume that $F_{\varepsilon} \rightarrow F_{0}(\varepsilon \rightarrow 0)$ locally uniformly and that $W_{\varepsilon} \rightarrow$ $W_{0}$ pointwise (so that $W_{\varepsilon}^{\prime \prime} \rightarrow W_{0}^{\prime \prime}$ weakly as measures) with $W_{\varepsilon}, W_{0} \in \mathcal{E}$. Let $u^{\varepsilon} \in C([0, \infty) \times \mathbf{T})$ be the generalized solution of

$$
\begin{cases}u_{t}+F_{\varepsilon}\left(u_{x}, \Lambda_{W_{s}}(u)\right)=0 & \text { in }(0, \infty) \times \mathbf{R}, \\ u(0, x)=u_{0}^{e}(x), & x \in \mathbf{R}\end{cases}
$$

with $u_{0}^{\varepsilon} \in C(\mathbf{T})$. If $u_{0}^{\varepsilon} \rightarrow u_{0}$ in $C(\mathbf{T})$ (i.e. $u_{0}^{\varepsilon}$ converges to $w_{0}$ uniformly in $\mathbf{T})$, then $u^{\varepsilon}$ converges to a function $u \in C([0, \infty) \times \mathbf{T})$ locally uniformly in $[0, \infty) \times \mathbf{T}$ and $u$ is the generalized solution of

$$
\begin{cases}u_{t}+F_{0}\left(u_{x}, \Lambda_{W_{0}}(u)\right)=0 & \text { in }(0, \infty) \times \mathbf{R} \\ u(0, x)=u_{0}(x), & x \in \mathbf{R}\end{cases}
$$

Note that $W^{\prime \prime}, W_{e}^{\prime \prime}$ may vanish somewhere and around these degenerate points no condition on degeneracy of $W^{\prime \prime}, W_{\varepsilon}^{\prime \prime}$ is imposed.

Of course every energy $W \in \mathcal{E}$ can be approximated by $W_{\varepsilon} \in C^{\infty}(\mathbf{R})$ with $W_{e}^{\prime \prime}>0$ so that $W_{\varepsilon} \rightarrow W(\varepsilon \rightarrow 0)$, and $u_{0} \in C(\mathbf{T})$ can be approximated by $u_{0}^{\varepsilon} \in C^{\infty}(\mathbf{T})$. We apply Theorem 1.1 with $F_{\varepsilon}=F_{0}, F_{0}(p, X)=-X$ in (1.7), (1.8) and conclude that the (smooth) solution $u^{\varepsilon}$ of (1.5),(1.6) does converge to the generalized solution $u$ of $(1.1),(1.4)$. This gives a way to construct the generalized solution $u$ of $(1.1),(1.4)$ and justifies our definition of generalized solution in [GG3].

By the way a solution of (1.1), (1.4) is also constructed in [FG] by adapting the theory of subdifferential equations because of the divergence structure of (1.1). Since their solution is also approximated by $u^{\varepsilon}$ as more special choice of $W_{e}$, a generalized solution in [GG3] based on the theory of viscosity solutions must agree with their solution if $u_{0} \in C(\mathrm{~T})$ [GG4].

In [S] the equation (1.1) with $W(p)=|p|^{2} / 2+c|p|, c>0$ was studied with a sine-function initial data. The problem was reduced to a free boundary problem in [FP] for the equation of $u_{x}$. Based on results in [FP] instant facet formation and finite time extinction of solution as well as unique existence of solutions were proved in [S], where the asymptotic behaviour near extinction time was also discussed. We are quite sure that the solution constructed in $[S]$ is a solution in our sense although we do not prove it explicitly here. In 
[GG3] instant facet formation and finite time extinction were proved for (1.1) with $W(p)=c|p|$ for a sine-function initial data by solving (1.1) explicitly.

If the graph of $u$ is regarded as a surface of crystal, equations (1.7) and (1.8) describe its evolution (at least for interface controlled model e.g. growth of Helium crystal). It is observed in physics [Ch] that $W^{\prime}$ has jumps if the temperature is low or precisely below the roughening temperature. A typical evolution equation is

$$
u_{t}-\left(1+u_{x}^{2}\right)^{1 / 2} f\left(u_{x}, \Lambda_{W}(u)\right)=0
$$

where $f=f(p, X)$ is continuous in its variables and nondecreasing in $X$ with $f(p, 0)=0$. Near $X=0$ it is often assumed that $f(p, X)$ is linear in $X$ provided that $p$ does not belong to the singularity $P$ of $W$ while the derivative $f_{X}(p, 0)=0$ for $p \in P$ because of structure of facets [Ch]. If $W$ and $f$ depend continuously on temperature, then our general convergence result says that evolution of crystal depends continuously on temperature even if facets appear. By the way in [S], $f(p, X)$ was assumed to be linear in $X$ even if for $p \in P$ so that $f(p, X)=0$ for $p \in P, X \in \mathbf{R}$. This prevents instant facet formation for the evaporation dynamics in $[S]$.

\subsection{Motion by crystalline energy.}

A typical example of an energy $W$ in $\mathcal{E}$ is a piecewise linear convex function. Such an energy is called a crystalline energy (density). For simplicity we assume that the singularity $P$ is a finite set, i.e.,

$$
P=\left\{p_{1}<p_{2}<\cdots<p_{m}\right\}
$$

To analyse (1.1) or more general form

$$
u_{t}-a\left(u_{x}\right)\left(\Lambda_{W}(u)-C\right)=0
$$

with $a \in C(\mathbf{R}), a>0$ and $C \in \mathbf{R}$, one often considers solutions in a special class of piecewise linear functions. Roughly speaking by our Convergence theorem 1.1 such a solution is also constructed as a limit of solutions of an approximate equation which is nondegenerate parabolic. To state this rigorously we recall a special class of piecewise linear solutions as in [GirK1] and [GG2]. 
For a piecewise linear continuous function $f$, a (bounded) closed (nontrivial) interval is called a faceted region of $f$ if it is a maximal interval on which $f$ is affine. We say that a piecewise linear continuous function is an admissible crystal if

(a) slope $f_{x}$ belongs to $P$;

(b) slope $f_{x}$ in adjacent faceted regions should be adjacent in $P$, i.e. if $f_{x}=p_{i}$ on a faceted region, then $f_{x}$ on an adjacent faceted region equals either $p_{i-1}$ or $p_{i+1}$ with $i+1 \leq m, i-1 \geq 1$.

We say that $u \in C(J \times \mathbf{T})$ is an admissible evolving crystal in a time interval $J$ if $u(t, \cdot)$ is an admissible crystal and (the abscissa of) a jump of $u_{x}(t, \cdot)$ moves smoothly in time $t \in J$. We have implicitly assumed that each jump does not collide each other. We now recall a system of ordinary differential equations (ODEs) so that an admissible evolving crystal solves (1.9). By periodicity of $u(t, x)$ in $x$ there are only finitely many points (for each $t \in J$ )

$$
\left\{x_{1}(t)<x_{2}(t)<\cdots<x_{d}(t)\right\}
$$

for which any faceted region of $u(t, \cdot)$ is represented as

$$
I_{j}(t)=\left[x_{j-1}(t), x_{j}(t)\right], \quad j=1, \cdots, d
$$

with convention that $x_{j}+\infty=x_{j+d}$. On each $I_{j}(t), u_{t}$ is independent of $x$ so its value is denoted $\left(u_{t}\right)_{j}(t)$ and $u_{x}$ is independent of both $x$ and $t$ so its value is denoted $\left(u_{x}\right)_{j}$. We say that an admissible evolving crystal $u \in C(J \times \mathbf{T})$ is an admissible solution of (1.9) if

$$
\left(u_{t}\right)_{j}(t)=a\left(\left(u_{x}\right)_{j}\right)\left(\frac{\chi_{j} \Delta\left(\left(u_{x}\right)_{j}\right)}{L_{j}(t)}-C\right), \quad j=1, \cdots, d, \quad t \in J
$$

with

$$
\begin{aligned}
& \Delta\left(p_{i}\right)=W^{\prime}\left(p_{i}+0\right)-W^{\prime}\left(p_{i}-0\right), \quad p_{i} \in P, \\
& L_{j}(t)=\text { the length of faceted region } I_{j}(t),
\end{aligned}
$$

where $\chi_{j}=1$ (resp. -1 ) if $u(t, \cdot)$ is convex (resp. concave) around $I_{j}$; otherwise $\chi_{j}=0$. The quantity $\Lambda_{W}(u)=\chi_{j} \Delta\left(\left(u_{x}\right)_{j}\right) / L_{j}(t)$ is nonlocal weighted curvature. An elementary geometric consideration shows that

$$
d L_{j}(t) / d t=\rho_{j}^{0}\left(u_{t}\right)_{j}+\rho_{j}^{-1}\left(u_{t}\right)_{j-1}+\rho_{j}^{1}\left(u_{t}\right)_{j+1}, \quad j=1, \cdots, d
$$


with

$$
\begin{aligned}
\rho_{j}^{0} & =\left(\left(u_{x}\right)_{j}-\left(u_{x}\right)_{j-1}\right)^{-1}+\left(\left(u_{x}\right)_{j+1}-\left(u_{x}\right)_{j}\right)^{-1}, \\
\rho_{j}^{-1} & =-\left(\left(u_{x}\right)_{j}-\left(u_{x}\right)_{j-1}\right)^{-1} \\
\rho_{j}^{1} & =-\left(\left(u_{x}\right)_{j+1}-\left(u_{x}\right)_{j}\right)^{-1} .
\end{aligned}
$$

Since $\chi_{j}$ and $\left(u_{x}\right)_{j}$ are time independent the system $(1.10),(1.11)$ yields a system of ODEs for $L_{j}$ 's so that its initial value problem is solvable locally in time. To simplify our description we assume that $a$ is positive on $P$. In a finite time some facet (with $\chi_{j}=0$ ) may disappear but fortunately even at that time $u(t, \cdot)$ stays as an admissible crystal so we solve (1.10) and (1.11) with less numbers of faceted regions (provided that $a>0$ ). We repeat this procedure and we eventually get $u \in C([0, \infty) \times \mathbf{T})$ which is an admissible solution on interval $\left[t_{k}, t_{k+1}\right)$ for $k=0, \cdots, h-1$ with some $t_{0}=0<t_{1}<t_{2}<\cdots<t_{h}$. Such a solution is called weakly admissible. As shown in [GG2] a weakly admissible solution is essentially a generalized solution. Let us summarize their results.

Lemma 1.2. Assume that $a \in C(\mathbf{R})$ is positive on $P$. Let $u_{0} \in C(\mathbf{T})$ be an admissible crystal. Then there is a unique weakly admissible solution $u \in C([0, \infty) \times \mathbf{T})$ of (1.9) with (1.4). The function $u$ is the generalized solution of (1.9) with (1.4) in the sense of [GG1-3], provided that $a$ is affine outside $P$ if $C \neq 0$.

For closed curves rather than graph-like functions, motion (1.9) by crystalline energy is first proposed by J. Taylor [T1] (see also [T2], [T3], [TCH]) (with $C=0$ and $a$ proportional to $W$ ) and independently by Angenent and Gurtin [AG] (see also [Gu]). They derived the ODE system corresponding to (1.10), (1.11). Admissibility at the time of disappearance of facets are observed by [T1]. For graph-like solutions the unique global existence of weakly admissible solution is shown in [GirK1-2] for the Dirichlet and Neumann problem (with $C=0$ ) and its extension to periodic case is rather straightforward. The consistency part of Lemma 1.2 is proved by [GG2] based on comparison principle in [GGu].

The speed of an admissible solution is determined nonlocally. Our convergence result says that weakly admissible solutions are obtained as a limit of solutions of nondegenerate parabolic equations. The next result is a trivial 
combination of Theorem 1.1 and Lemma 1.2.

Corollary 1.3 (Motion by crystalline energy as a limit of that of smooth strictly convex energy). Let $u^{\varepsilon}$ be a classical solution of nondegenerate parabolic equation

$$
u_{t}-a^{\varepsilon}\left(u_{\infty}\right)\left(\Lambda_{W_{c}}(u)-C\right)=0 \quad \text { in }(0, \infty) \times \mathbf{R}
$$

with initial data $u_{0}^{\varepsilon} \in C^{\infty}(\mathbf{T})$ where $a^{\varepsilon}, W_{\varepsilon} \in C^{\infty}(\mathbf{R})$ with $a^{\varepsilon}>0$ and $W_{\varepsilon}^{\prime \prime}>0$ with $W_{\varepsilon} \rightarrow W(\varepsilon \rightarrow 0)$ pointwise. Assume that $a^{\varepsilon} \rightarrow$ a locally uniformly and that $a$ is positive on the singularity $P$ of $W$. Let $u_{0} \in C(\mathrm{~T})$ be an admissible crystal. If $u_{0}^{\varepsilon} \rightarrow u_{0}(\varepsilon \rightarrow 0)$ in $C(\mathbf{T})$, then $u^{\varepsilon}$ converges to the weakly admissible solution $u$ of (1.9), (1.4) locally uniformly in $[0, \infty) \times \mathbf{T}$ as $\varepsilon \rightarrow 0$ provided that $a$ is affine outside singularity $P$ of $W$ if $C \neq 0$.

As for (1.5), (1.6) the existence of classical solution for (1.12), (1.6) is standard. For crystalline $W$ it is easy to find approximations $W_{e}$ and $u_{0}^{\varepsilon}$ so a weakly admissible solution is actually constructed as a limit of solutions of nondegenerate parabolic equation.

\subsection{Approximation by crystalline algorithm.}

It is well known that for (1.1) and (1.9) with $C=0$ motion by crystalline energy approximates motion by smooth energy [GirK1-2], [Gir] and [FG]. Since a weakly admissible solution is computable by solving the system of ODEs (1.10)-(1.11), this gives a numerical way to calculate an approximate solution of (1.9) with general $W$. Our Convergence theorem 1.1 justifies this idea for equation (1.9). We shall give a simple way of approximation (which is by no means optimal). For given energy $W \in \mathcal{E}$ and $\varepsilon>0$ we take finitely many points

$$
P_{\varepsilon}=\left\{q_{1}<\cdots<q_{m}\right\}
$$

with $m \geq 1 / \varepsilon^{2}, \max _{1 \leq j \leq m-1}\left|q_{j+1}-q_{j}\right| \leq \varepsilon, q_{1} \leq-1 / \varepsilon, q_{m} \geq 1 / \varepsilon$ and a piecewise linear (convex) function $W_{\varepsilon}(p)$

$$
W_{\varepsilon}(p)= \begin{cases}W\left(q_{j}\right) & \text { for } p=q_{j}, j=1, \cdots, m \\ \text { affine } & \text { otherwise }\end{cases}
$$


By this choice $W_{\epsilon}$ converges to $W$ (locally uniformly). Of course it is possible to choose such $P_{\varepsilon}$. Indeed, if we take

$$
P_{e}=\left\{j \varepsilon ; j=0, \pm 1, \pm 2, \cdots, \pm m, m=\left[1 / \varepsilon^{2}\right]+1\right\}
$$

then this $P_{\varepsilon}$ satisfies all required conditions. Here [ $\left.\cdot\right]$ denotes the integer part. We assume that $W$ is strictly convex so that $W_{e}^{\prime}$ always jumps at $q_{j}$ 's; this assumption is just for simplicity.

Theorem 1.4 (Approximation by crystalline algorithm). Let $W \in \mathcal{E}$ be strictly convex and $a \in C(\mathbf{R})$ be positive. Let $W_{c}$ be as in (1.13). For given $u_{0} \in C(\mathbf{T})$ let $u_{0}^{\varepsilon} \in C(\mathbf{T})$ be an admissible crystal with respect to $W_{\varepsilon}$ and assume that $u_{0}^{\varepsilon} \rightarrow u_{0}$ in $C(\mathrm{~T})$ as $\varepsilon \rightarrow 0$. Assume that $a^{e}$ agrees with a at singularities of $W_{\varepsilon}$. Then the weakly admissible solution $u^{\varepsilon} \in C([0, \infty) \times \mathbf{T})$ of (1.12), (1.6) converges to the (generalized) solution of (1.9) with (1.4) locally uniformly in $[0, \infty) \times \mathbf{T}$ as $\varepsilon \rightarrow 0$.

It is possible to approximate $u_{0}$ by $W_{\epsilon}$-admissible crystal $u_{0}^{\varepsilon}$ at least for special choice of approximation, so this result yields an algorithm to calculate approximate solutions of (1.9), (1.4) numerically [GirK1]. Theorem 1.4 follows from Theorem 1.1, since $u^{\varepsilon}$ is the generalized solution of $(1.12),(1.6)$ if $a^{\varepsilon} \in C(\mathbf{R})$ is taken to be affine outside the singularity of $W^{\varepsilon}$ by Lemma 1.2 ; by this choice of $a^{\varepsilon}$ evidently $a^{\varepsilon} \rightarrow a$ is locally uniform.

In $[\mathrm{FG}]$ the convergence is proved in $C\left([0, T), L^{2}(\mathbf{T})\right)$ for (1.1) or (1.9) with $C=0$ without assuming strictly convexity of $W$. The paper [GirK1] is interesting since the uniform convergence rate with respect to $\varepsilon$ is also proved for (1.1),(1.9) with $C=0$ under the Dirichlet and Neumann boundary conditions, when $W$ is $C^{3}$ and $W^{\prime \prime}>0$ so that the problem (1.1) admits regular solutions. For closed convex curves see also [Gir]. There is a nice review [GirK2] on crystalline algorithm with other numerical methods. Recently, Ushijima and Yazaki announced that they extended results in [Gir] for the motion by a power of curvature. Our result is quite general but it does not give any convergence rate.

It turns out that the derivative $u_{x}^{e}(t, \cdot)$ converges to $u_{x}(t, \cdot)$ for all $t \geq 0$ in $L^{r}(\mathbf{T})(1 \leq r<\infty)$ in Theorem 1.1 on convergence provided that $\left\|u_{0 x \boldsymbol{x}}^{\varepsilon}\right\|_{1}$, the total variation of the measure $u_{0 x x}^{\varepsilon}$ is bounded for $0 \leq \varepsilon<1$. This follows 
from

$$
\left\|u_{x x}^{\varepsilon}(t, \cdot)\right\|_{1} \leq\left\|u_{0 x x}^{\varepsilon}\right\|_{1} \text {. }
$$

Such an estimate formally follows from the $L^{1}$ contraction property of the equation of $v=u_{x}$ :

$$
v_{t}+\partial_{x}\left(F_{\varepsilon}\left(v, W_{\varepsilon}^{\prime}(v)_{x}\right)=0\right.
$$

obtained by differentiating (1.7) in $x$. We shall discuss the convergence problem on derivatives in detail as well as related references in our forthcoming papers and we do not pursue this subject in this paper.

\subsection{Method of the proof.}

The proof of Theorem 1.1 is based on the stability property of our generalized solution. This property is usually expected for viscosity solutions.

Theorem 1.5 (Stability). Assume that same hypotheses of Theorem 1.1 concerning $F_{\varepsilon}, F_{0}(=F), W_{\varepsilon}, W_{0}(=W)$. Let $u^{\varepsilon}$ be a subsolution of

$$
u_{t}+F_{\varepsilon}\left(u_{x}, \Lambda_{W_{*}}(u)\right)=0
$$

in $Q=(0, T) \times \Omega$ with an open interval $\Omega$. Then the uppersemicontinuous limit $\bar{u}=\lim \sup ^{*} u^{\varepsilon}$ is a subsolution of (1.2) in $Q$ provided that $\bar{u}<\infty$ in $[0, T) \times \bar{\Omega}$.

More general version is stated in Theorem 2.9. If $W_{\varepsilon}$ and $W_{0}$ are $C^{2}$, this is a special case of stability theorems of viscosity solutions due to Barles and Perthame $[\mathrm{BaP} 1,2],[\mathrm{Ba}]$ and Ishii [I]. Let us briefly review their idea. It suffices to prove

$$
\psi_{t}(\hat{t}, \hat{x})+F\left(\psi_{x}(\hat{t}, \hat{x}), \Lambda_{W}(\psi(\hat{t}, \cdot)(\hat{x})) \leq 0\right.
$$

at $(\hat{t}, \hat{x}) \in Q$ for a test function $\psi \in C^{2}(Q)$ of $\bar{u}($ at $(\hat{t}, \hat{x}))$ i.e. $\bar{u}-\psi$ takes its maximum over $Q$ at $(\hat{t}, \hat{x})$. By a standard argument we may assume that the maximum point $\left(t_{\varepsilon}, x_{\varepsilon}\right)$ of $u^{\varepsilon}-\psi$ converges to $(\hat{t}, \hat{x})$ by taking a subsequence. Since $u^{\varepsilon}$ is a subsolution of (1.14) one obtains

$$
\psi_{t}\left(t_{\varepsilon}, x_{\varepsilon}\right)+F_{\varepsilon}\left(\psi_{x}\left(t_{\varepsilon}, x_{\varepsilon}\right), \Lambda_{W}\left(\psi\left(t_{\varepsilon}, \cdot\right)\right)\left(x_{\varepsilon}\right)\right) \leq 0
$$

which yields (1.15) as $\varepsilon \rightarrow 0$. 
In our problem we are forced to choose test functions depending on $\varepsilon$ because the class of test functions depends on $\varepsilon$. For this purpose we introduce a Wulff function $w^{e}$ which is obtained by rescaling the convex conjugate of $W_{e}$ so that $W_{e}$ - weighted curvature of $w^{e}$ is constant (say $\mu$ ) independent of $x$ and $\varepsilon>0$ at least formally. We take such a Wulff function $w^{\varepsilon}$ to modify the spatial part $\psi(\hat{t}, x)$ of $\psi$ and get $\psi^{e}$ which approximates $\psi$ with

$$
\Lambda_{W}\left(\psi^{e}\left(t_{\varepsilon}, \cdot\right)\right)=\mu \doteqdot \Lambda_{W}(\psi(\hat{t}, \cdot))
$$

If $\psi^{\varepsilon}$ is regarded as a test function of $u^{\varepsilon}$, we obtain

$$
\psi_{t}^{\varepsilon}\left(t_{\varepsilon}, x_{\varepsilon}\right)+F_{\varepsilon}\left(\psi_{x}^{\varepsilon}\left(t_{\varepsilon}, x_{\varepsilon}\right), \mu\right) \leq 0
$$

instead of $(1.16)$, where $\left(t_{\varepsilon}, x_{\varepsilon}\right)$ is a maximum point of $u^{\varepsilon}-\psi^{\varepsilon}$. Sending $\varepsilon$ to zero in (1.17) yields (1.15). Unfortunately because of the lack of regularity of $w^{\varepsilon}$ we have to show that $\psi^{\varepsilon}$ plays a role of a test function of $u^{\varepsilon}$. This requires an extra work. The idea of $\varepsilon$ dependent test functions was previously used for homogenization problem [E] but our choice is evidently different from those in $[E]$ since the problem is different.

Once stability result is proved, then convergence result can be proved without using estimates of derivatives [Ba]. Since the lowersemicontinuous limit $\underline{u}$ is a supersolution by stability, if initially $\bar{u} \leq \underline{u}$, from the comparison (Theorem 2.7) it follows that $\bar{u}=\underline{u}$ which yields the convergence. To show $\bar{u} \leq \underline{u}$ at $t=0$ we use Wulff functions as barriers.

In section 2, we review our definitions of solutions as well as other properties of solutions and state our main results. In section 3 we give a sufficient condition for sub- and supersolutions. In section 4 Wulff functions are introduced. Since a Wulff function is not $C^{2}$ in general we need to check whether it can play a role of admissible test functions. This step becomes technical since we impose no conditions on degeneracy of $W^{\prime \prime}$. Sometimes the domain of definition of Wulff functions is not necessarily $\mathbf{R}$ so we modify it so that it is defined everywhere in $\mathbf{R}$. In section 5 we observe that if $W_{e}^{\prime \prime} \rightarrow W^{\prime \prime}$ as measures as $\varepsilon \rightarrow 0$, then the Wulff functions of $W_{\epsilon}$ converge that of $W$. 
In section 6 we prove our general stability theorem. The convergence results are proved in section 7. In Appendix we compare various convergences.

The bibliography of [GG3] includes many references to recent work on the motion by crystalline energy and nonsmooth energy. We take this opportunity to note some other, related article not cited there and not mentioned elsewhere in this introduction. In Theorem 1.1 the local finiteness of total variation of $W^{\prime \prime}$ is essential. Even for $(1.1)$ if $W^{\prime \prime}(p)=1 /|p|^{\sigma}$ with $\sigma \geq 1$ as shown in [CGS] the limit of approximate solution may not fulfill the initial condition. In [GG4] a special version of convergence results in the present paper is announced. There several solutions of (1.9) when $a \equiv 1$ but $C$ depends on space variable are studied especially for piecewise linear $W$. It turns out that some facet should be split at some time as suggested in $[R]$. Although, we hope we extend our approach to this case, the notion of admissible solution should be quite different from the case where $C$ is independent of the space variable. As mentioned before the crystalline algorithm provides numerical solutions for the curvature flow problem. A somewhat related scheme but different was proposed by Robert [Rob]. There are nice review [El] on numerical methods for the curvature flow problem including crystalline method. After this work was completed, we were informed of a recent work of $\mathrm{K}$. Ishii and Soner [ISo] related to ours. They proved the convergence of polygonal evolution moved by crystalline curvature to the motion by isotropic curvature in the plane when the length of curves is approximated by its crystalline energy. Although their method is regarded as a special case of ours, they rather treated closed curves than graph-like functions.

\section{§2. Definition and main results.}

Throughout this paper $\Omega$ denotes an open interval. We begin by recalling the notion of solutions defined in [GG3].

Definition 2.1 (Class of convex functions). Let $W$ be a real valued convex function on $\mathbf{R}$. Let $P$ denote the set of points $p$ at which $W$ is not $C^{2}$. We say $W$ belongs to class $\mathcal{E}$ if $P$ is discrete and $W^{\prime \prime}$ is bounded in any compact set except all points in $P$. The set $P$ is called the singularity of $W$. The space $\mathcal{A}$ consists of all second derivatives $W^{\prime \prime}$ (in distribution sense) of $W$ belonging to $\mathcal{E}$. 
Since $\mathcal{E}$ is a subset of the space of convex functions, $\mathcal{A}$ is a subset of the space of nonnegative Radon measures on $\mathbf{R}$. By definition $\mathcal{A}$ consists of nonnegative Radon measures of the form

$$
\alpha(q)=\sum_{p \in P} \Delta(p) \delta(q-p)+\alpha^{c}(q) .
$$

Here $P$ is the discrete set of points $p$ at which either $\Delta(p)>0$, or $\alpha^{c}$ is not continuous at $p$ and $\alpha^{c}$ fulfills

$$
\begin{aligned}
& \alpha^{c} \in C(\mathbf{R} \backslash P) \quad \text { and } \\
& \sup \left\{\alpha^{c}(q) ; q \in K \backslash P\right\}<\infty
\end{aligned}
$$

for every compact set $K$ in $\mathbf{R}$. For $\alpha \in \mathcal{A}$, there is $W \in \mathcal{E}$ that satisfies $\alpha=W^{\prime \prime}$. Such $W$ is uniquely determined by $\alpha$ up to addition of affine functions. If $\alpha=W^{\prime \prime}$ is of the form, evidently $\Delta(p)$ is the jump of $W^{\prime}$ at $p \in P$, i.e.

$$
\Delta(p)=W^{\prime}(p+0)-W^{\prime}(p-0) .
$$

By our definition $\Delta(p)$ may be zero at $p \in P$. At such a point $p, W$ is $C^{1}$ but not $C^{2}$.

Definition 2.2 ( $P$-faceted). Let $P$ be a discrete set in $\mathbf{R}$. Let $\Omega$ be an open interval. A function $f$ in $C(\Omega)$ is called faceted at $x_{0} \in \Omega$ with slope $p$ in $\Omega$ if $f$ fulfills the following conditions.

There are a closed nontrivial finite interval $I(\subset \Omega)$ containing $x_{0}$ such that $f$ agrees with an affine function

$$
\ell_{p}(x)=p\left(x-x_{0}\right)+f\left(x_{0}\right)
$$

in $I$ and $f(x) \neq \ell_{p}(x)$ for all $x \in J \backslash I$ with some neighborhood $J(\subset \Omega)$ of $I$. The interval $I$ is called a faceted region of $f$ containing $x_{0}$ and is denoted by $R\left(f, x_{0}\right)$.

A function $f$ is called $P$-faceted at $x_{0}$ in $\Omega$ if $f$ is faceted at $x_{0}$ in $\Omega$ with some slope $p$ belonging to $P$.

Definition 2.3 (Weighted curvature). For $W \in \mathcal{E}$ let $P$ be the singularity of $W$. Let $x_{0}$ be a point in $\Omega$. For $f \in C(\Omega)$ we set the value

$$
\Lambda_{W}\left(f, x_{0}\right)=W^{\prime \prime}\left(f^{\prime}\left(x_{0}\right)\right) f^{\prime \prime}\left(x_{0}\right)
$$


if $f$ is second differentiable at $x_{0}$ and $f^{\prime}\left(x_{0}\right) \notin P$ and

$$
\Lambda_{W}\left(f, x_{0}\right)=\frac{\chi}{L} \Delta(p)
$$

if $f$ is $P$-faceted at $x_{0}$ in $\Omega$ with slope $p$, where $\Delta(p)$ is given by (2.4). If $x_{0}$ is on the boundary of two faceted region $R\left(f, x_{0}\right)$ and $R\left(f, x_{1}\right)$ for some $x_{1}$, i.e., $x_{0} \in \partial R\left(f, x_{0}\right) \cap \partial R\left(f, x_{1}\right)$, then our definition gives two values for $\Lambda_{W}\left(f, x_{0}\right)$. To avoid this inconvenience, we assume that $f$ is $C^{1}$ at $x_{0}$ to define $\Lambda_{W}\left(f, x_{0}\right)$ for $x_{0} \in \partial R\left(f, x_{0}\right)$. We call the value $\Lambda_{W}\left(f, x_{0}\right)$ weighted curvature of $f$ at $x_{0}$. This value is invariant under the addition of affine functions to $W$, so it is determined by $a=W^{\prime \prime} \in \mathcal{A}$. Here $L=L\left(f, x_{0}\right)$ is the length of the faceted region $I$ containing $x_{0}$ and $\chi=\chi\left(f, x_{0}\right)$ is the transition number defined by

$$
\begin{cases}\chi=+1 & \text { if } f \geq \ell_{p_{i}} \quad \text { in } \quad J \\ \chi=-1 & \text { if } f \leq \ell_{p_{i}} \quad \text { in } \quad J \\ \chi=0 & \text { otherwise }\end{cases}
$$

for some neighborhood $J$ of the faceted region $I$. We often write $\Lambda_{W}\left(f, x_{0}\right)$ by $\Lambda_{W}(f)\left(x_{0}\right)$ if we emphasize that this quantity is a function of $x_{0}$.

For later convenience we also recall the left transition number $\chi_{-}=$ $\chi_{-}\left(f, x_{0}\right)$, and the right transition number $\chi_{+}=\chi_{+}\left(f, x_{0}\right)$ by

$$
\left\{\begin{array}{l}
\chi_{-}=+1 \text { if } f \geq \ell_{p_{i}} \text { in }\left\{x \in J ; x \leq x_{0}\right\} \\
\chi_{-}=-1 \text { if } f \leq \ell_{p_{i}} \text { in }\left\{x \in J ; x \leq x_{0}\right\}
\end{array}\right.
$$

and

$$
\left\{\begin{array}{l}
\chi_{+}=+1 \text { if } f \geq \ell_{p_{i}} \text { in }\left\{x \in J ; x \geq x_{0}\right\} \\
\chi_{+}=-1 \text { if } f \leq \ell_{p_{i}} \text { in }\left\{x \in J ; x \geq x_{0}\right\}
\end{array}\right.
$$

respectively. By definition we see $\chi=\left(\chi_{+}+\chi_{-}\right) / 2$.

Definition 2.4 (Space of admissible functions). A function $f \in C^{2}(\Omega)$ belongs to a class $C_{P}^{2}(\Omega)$ if $f$ is $P$-faceted at $x_{0}$ in $\Omega$ whenever $f^{\prime}\left(x_{0}\right)$ belongs to $P$. Let $T$ be a positive number. For $Q=(0, T) \times \Omega$ with $T>0$ let $A_{P}(Q)$ be the set of functions on $Q$ of the form

$$
f(x)+g(t), \quad f \in C_{P}^{2}(\Omega), \quad g \in C^{1}(0, T) .
$$


An element of $A_{P}(Q)$ is called an admissible function. Note that for given $\psi \in A_{P}(Q)$ this decomposition is unique up to an additive constant.

For $f \in C_{P}^{2}(\Omega)$ if $f$ is $P$-faceted at $x_{0}$ and $x_{0} \in \partial R\left(f, x_{0}\right)$, then $\Lambda_{W}\left(f, x_{0}\right)$ is the value (2.6) not necessarily zero although $f^{\prime \prime}\left(x_{0}\right)=0$. Although we do not use the value of weighted curvature at the boundary of faceted region in this paper, this way of definition is convenient to show the fact that the notion of subsolution is closed under the operation of supremum [GG3].

Definition 2.5 (Class of nonlinearity). Let $F$ be a function from $[0, T) \times$ $\mathbf{R} \times \mathbf{R}$ to $\mathbf{R}$. We say that $F$ belongs to class $\mathcal{F}$ if $F$ fulfills the following two conditions:

(F1) $F$ is continuous on $[0, T) \times \mathbf{R} \times \mathbf{R}$ with values in $\mathbf{R}$,

(F2) $F$ is degenerate elliptic in the sense that $F(t, p, X) \leq F(t, p, Y)$ for all $X \geq Y, t \in[0, T), p \in \mathbf{R}$.

If $F \in \mathcal{F}$ further fulfills

(F3) $F$ is either independent of $t$ or for each $T^{\prime}<T$ and $K>0$ uniformly continuous on $\left[0, T^{\prime}\right] \times[-K, K] \times \mathbf{R}$,

then we say that $F$ belongs to class $\mathcal{F}_{\#}$.

We now recall definition of generalized solution in the viscosity sense [GG3].

Definition 2.6 (Generalized solution). Assume that $F \in \mathcal{F}$ and that $W \in \mathcal{E}$ with singularity $P$. A real valued function $u$ on $Q$ is a (viscosity) subsolution of

$$
u_{t}+F\left(t, u_{x}, \Lambda_{W}(u)\right)=0 \quad \text { in } Q=(0, T) \times \Omega
$$

if the uppersemicontinuous envelope $u^{*}<\infty$ in $[0, T) \times \bar{\Omega}$ and

$$
\psi_{t}(\hat{t}, \hat{x})+F\left(t, \psi_{x}(\hat{t}, \hat{x}), \Lambda_{W}(\psi(\hat{t}, \cdot), \hat{x})\right) \leq 0
$$

whenever $(\psi,(\hat{t}, \hat{x})) \in A_{P}(Q) \times Q$ fulfills

$$
\max _{Q}\left(u^{*}-\psi\right)=\left(u^{*}-\psi\right)(\hat{t}, \hat{x})
$$


Here

$$
u^{*}(t, x)=\lim _{\varepsilon \downarrow 0} \sup \{u(s, y) ;|s-t|<\varepsilon,|x-y|<\varepsilon,(s, y) \in Q\}
$$

for $(t, x) \in \bar{Q}$ and $u_{*}=-(-u)^{*}$. A (viscosity) supersolution is defined by replacing $u^{*}(<\infty)$ by the lowersemicontinuous envelope $u_{*}(>-\infty)$, max by $\mathrm{min}$ and the inequality in (2.7) by the reverse one. If $u$ is sub- and supersolution, $u$ is called a viscosity solution or a generalized solution. Hereafter we avoid to use the word viscosity. Function $\psi$ satisfying $(2.8)$ is called a test function of $u$ at $(\hat{t}, \hat{x})$.

\subsection{Fundamental known results.}

We list results from [GG3] only for periodic data for later use.

Theorem 2.7 (Comparison). Assume that $F \in \mathcal{F}_{\#}$ and $W \in \mathcal{E}$. Let $u$ and $v$ be a sub-, and supersolution of $(E)$ in $(0, T) \times \mathbf{R}$, respectively. Suppose that $u$ and $v$ are periodic in the spatial variable $z$ with period $\varpi$. If $u^{*} \leq v_{*}$ on $\{0\} \times \mathbf{R}$, then $u^{*} \leq v_{*}$ in $(0, T) \times \mathbf{R}$.

Theorem 2.8 (Existence). Assume that $F \in \mathcal{F}_{\#}$ and $W \in \mathcal{E}$. Suppose that $u_{0} \in C(\mathbf{R})$ is periodic with period $\varpi$. Then there exists a unique function $u \in C([0, T) \times \mathbf{R})$ that satisfies

$$
u \text { is a generalized solution of }(E) \text { in }(0, T) \times \mathbf{R} \text {, }
$$

$$
\begin{aligned}
& u(0, x)=u_{0}(x) \quad \text { for } x \in \mathbf{R}, \\
& u(t, x+\varpi)=u(t, x) \quad \text { for }(t, x) \in[0, T) \times \mathbf{R} .
\end{aligned}
$$

If $u_{0}$ is Lipschitz with constant $L$ i.e.,

$$
\left|u_{0}(x)-u_{0}(y)\right| \leq L|x-y|
$$

for all $x, y \in \mathbf{R}$, then

$$
|u(t, x)-u(t, y)| \leq L|x-y|
$$

for all $\boldsymbol{x}, y \in \mathbf{R}, 0 \leq t<T$.

\subsection{Stability.}


We consider a family of equations

$\left(\mathrm{E}_{\varepsilon}\right)$

$$
u_{t}+F_{\varepsilon}\left(t, u_{x}, \Lambda_{W_{s}}(u)\right)=0 \quad \text { in } Q=(0, T) \times \Omega
$$

with $\varepsilon \geq 0$ where $F_{e} \in \mathcal{F}$ and $W_{\varepsilon} \in \mathcal{E}$. We are asking whether solution $u^{\varepsilon}$ of $\left(\mathrm{E}_{\varepsilon}\right)$ converges to a solution of $\left(\mathrm{E}_{0}\right)$ if $F_{\varepsilon}$ tends to $F_{0}$ and $W_{\varepsilon}$ tends to $W_{0}$ as $\varepsilon \rightarrow 0$. Fortunately, as one of main results we have a general stability theorem, which is more general than Theorem 1.5.

Theorem 2.9 (General stability). Assume that $F_{\varepsilon} \in \mathcal{F}$ converges to $F_{0} \in$ $\mathcal{F}$ locally uniformly in $(0, T) \times \mathbf{R} \times \mathbf{R}$ as $\varepsilon \rightarrow 0$. Assume that $\alpha_{\varepsilon}=W_{\varepsilon}^{\prime \prime} \in \mathcal{A}$ converges to $\alpha_{0}=W_{0}^{\prime \prime} \in \mathcal{A}$ weakly as measures as $\varepsilon \rightarrow 0$ (with $W_{\varepsilon}, W_{0} \in \mathcal{E}$ ). Let $u^{e}$ be a subsolution (resp. supersolution) of $\left(E_{\varepsilon}\right)$ in $Q$ for $\varepsilon>0$, where $Q=(0, T) \times \Omega$ with an open interval $\Omega$ (not necessarily bounded). Let $\bar{u}$ (resp. $\underline{u}$ ) be the upper (resp. lower) semicontinuous limit of $u^{\varepsilon}$, i.e.,

$$
\begin{aligned}
& \bar{u}(t, x)=\left(\limsup _{\varepsilon \rightarrow 0}^{*} u^{\varepsilon}\right)(t, x) \\
& =\lim _{\varepsilon \rightarrow 0} \sup \left\{u^{\delta}(s, y) ;|s-t|<\varepsilon,|y-x|<\varepsilon, 0<\delta<\varepsilon,(s, y) \in Q\right\} \\
& \underline{u}=\liminf _{e \rightarrow 0} u^{e}=-\limsup _{\varepsilon \rightarrow 0}^{*}\left(-u^{e}\right) .
\end{aligned}
$$

If $\bar{u}(t, x)<\infty$ (resp. $\underline{u}(t, x)>-\infty)$ for $(t, x) \in[0, T) \times \bar{\Omega}$, then $\bar{u}$ (resp. $\underline{u}$ ) is a subsolution (resp. supersolution) of $\left(E_{0}\right)$ in $Q$.

Remark 2.10. As we see in Appendix if $W_{\varepsilon} \rightarrow W_{0}$ pointwise (as $\varepsilon \rightarrow 0$ ), then $\alpha_{\varepsilon} \rightarrow \alpha_{0}$ weakly as measures so we may replace the assumption $\alpha_{\varepsilon} \rightarrow \alpha_{0}$ by $W_{e} \rightarrow W_{0}$ pointwise.

\subsection{Convergence.}

We now consider the initial value problem for $\left(\mathrm{E}_{\varepsilon}\right)$ for periodic initial data. We state our main results (which is more general than Theorem 1.1) on convergence of solutions.

Theorem 2.11 (Convergence with periodic boundary condition). Assume that $F_{\varepsilon} \in \mathcal{F}_{\#}$ converges to $F_{0} \in \mathcal{F}_{\#}$ locally uniformly in $[0, T) \times \mathbf{R} \times$ $\mathbf{R}$ as $\varepsilon \rightarrow 0$. Assume that $\alpha_{\varepsilon}=W_{\varepsilon}^{\prime \prime} \in \mathcal{A}$ converges to $\alpha_{0}=W_{0}^{\prime \prime} \in \mathcal{A}$ weakly as measures as $\varepsilon \rightarrow 0$ (with $W_{\varepsilon}, W_{0} \in \mathcal{E}$ ). For $\varepsilon>0$ let $u^{\varepsilon} \in C([0, T) \times \mathbf{R}$ ) be 
the generalized solution (obtained in Theorem 2.8) of $\left(E_{\varepsilon}\right)$ with initial data $u_{0}^{\varepsilon} \in C(\mathbf{R})$, where $u_{0}^{\varepsilon}$ is periodic with period $\varpi$ (i.e. $\varpi$-periodic) independent of $\varepsilon>0$. Suppose that $u_{0}^{\varepsilon}$ converges to a function $u_{0}$ uniformly in $\mathbf{R}$ (so that $\left.u_{0} \in C(\mathbf{R})\right)$ as $\varepsilon \rightarrow 0$. Then $u^{\varepsilon}$ converges to some function $u \in C([0, T) \times \mathbf{R})$ locally uniformly in $[0, T) \times \mathbf{R}$ as $\varepsilon \rightarrow 0$ and $u$ is the generalized solution of $\left(E_{0}\right)$ in $(0, T) \times \mathbf{R}$ with initial data $u_{0}$.

Remark 2.12 (Dirichlet and Neumann boundary conditions). The convergence Theorem 2.11 applies to homogeneous Dirichlet and Neumann boundary value problems under structural hypotheses on $F_{\varepsilon}$. To carry out we need similar reflection argument as in the proof of [GG3, Theorem 3.6, Remark 3.8]. We state results for the Dirichlet problem only for the reader's convenience.

Theorem 2.13 (Dirichlet condition). Assume the same hypotheses of Theorem 2.11 concerning on $F_{\varepsilon}$ and $W_{e}$. Assume that

$$
F_{e}(t, p, X)=-F_{e}(t, p,-X)
$$

for $\varepsilon \geq 0$. Let $\Omega$ be a bounded open interval. Let $u^{\varepsilon} \in C([0, T) \times \bar{\Omega})$ be the generalized solution of $\left(E_{\varepsilon}\right)$ on $Q=(0, T) \times \Omega$ with $u^{\varepsilon}(t, x)=0$ for $x \in \partial \Omega, t \in[0, T)$ and $u^{\varepsilon}(0, x)=u_{0}^{\varepsilon}(x) \in C(\bar{\Omega})$ (with $u_{0}^{\varepsilon}=0$ on $\partial \Omega$ ). Suppose that $u_{0}^{\varepsilon}$ converges to a function $u_{0}$ uniformly in $\bar{\Omega}$ (so that $u_{0} \in C(\bar{\Omega}$ ) with $u_{0}=0$ on $\left.\partial \Omega\right)$. Then $u^{\varepsilon}$ converges to a function $u \in C([0, T) \times \bar{\Omega})$ locally uniformly in $[0, T) \times \bar{\Omega}$ as $\varepsilon \rightarrow 0$ and $u$ is the generalized solution of $\left(E_{0}\right)$ in $(0, T) \times \Omega$ with $u=0$ on $[0, T) \times \partial \Omega$ and $u(0, x)=u_{0}(x)$ for $x \in \bar{\Omega}$.

We give a typical situation our convergence results apply.

Theorem 2.14. Assume that $a^{\varepsilon} \in C(\mathbf{R})$ converges to $a^{0} \in C(\mathbf{R})$ locally uniformly in $\mathbf{R}$ as $\varepsilon \rightarrow 0$ and that $a^{c}, a^{0} \geq 0$. Assume that $W_{e} \in \mathcal{E}$ converges to $W_{0} \in \mathcal{E}$ pointwise (so that $a^{\varepsilon}=W_{e}^{\prime \prime} \rightarrow a^{0}=W_{0}^{\prime \prime}$ weakly as measures as remarked in Remark 2.10). Assume that $C_{e} \in C[0, T)$ converges to $C_{0} \in$ $C[0, T)$ locally uniformly in $[0, T)$.

(i) (Periodic case) Let $u^{\varepsilon} \in C([0, T) \times \mathbf{R})$ be the generalized solution of

$$
u_{t}-a^{e}\left(u_{x}\right)\left(\Lambda_{W_{\varepsilon}}(u)-C_{\varepsilon}\right)=0
$$


in $(0, T) \times \mathbf{R}$ with initial condition $u^{\varepsilon}(0, x)=u_{0}^{e}(x)$, where $u_{0}^{e}$ is $\varpi$-periodic for $\varepsilon>0$. Assume that $u_{0}^{e}$ converges to $u_{0}$ uniformly in $\mathbf{R}$. Then $u^{\varepsilon}$ converges to $u \in C([0, T) \times \mathbf{R})$ locally in uniformly $[0, T) \times \mathbf{R}$ and $u$ is the generalized solution of

$$
u_{t}-a^{0}\left(u_{x}\right)\left(\Lambda_{W_{0}}(u)-C_{0}\right)=0
$$

in $[0, T) \times \mathbf{R}$ with $\varpi$-periodic initial data $u_{0}$.

(ii) (Dirichlet problem) Let $\Omega$ be a bounded open interval and assume that for $\varepsilon>0, u^{\varepsilon} \in C([0, T) \times \bar{\Omega})$ be the generalized solution of

$$
u_{t}-a^{e}\left(u_{x}\right) \Lambda_{W_{s}}(u)=0
$$

in $(0, T) \times \Omega$ with $u=0$ on $[0, T) \times \partial \Omega$ and $u(0, x)=u_{0}^{\varepsilon}(x) \in C(\bar{\Omega})$ with $u_{0}^{\varepsilon}=0$ on $\partial \Omega$. Assume that $u_{0}^{\varepsilon}$ converges to $u_{0}$ uniformly in $\bar{\Omega}$. Then $u^{\varepsilon}$ converges to some function $u \in C([0, T) \times \bar{\Omega})$ locally uniformly in $[0, T) \times \bar{\Omega}$ and $u$ is the generalized solution of

$$
u_{t}-a^{0}\left(u_{x}\right) \Lambda_{W_{0}}(u)=0
$$

with $u(t, x)=0$ for all $x \in \partial \Omega, t \in[0, T)$ and $u(0, x)=u_{0}(x)$ for $x \in \bar{\Omega}$.

Remark. For equations (2.11) and (2.12) the stability results for nonlinear semigroups in a Hilbert space apply for periodic boundary value problems [FG]. However, such theory only provides convergence $u^{e} \rightarrow u$ in $C([0, T)$; $L^{2}(\mathbf{R} / \varpi \mathbf{Z})$ ) but not uniform convergence. Although our solution operator $u_{0}^{e} \rightarrow u_{\varepsilon}(t, \cdot)$ provides a contraction semigroup on $C(\mathbf{R} / \varpi \mathbf{Z})$ [GG4], it seems that the standard stability results by Brezis-Pazy [BP] does not apply since the domain of generator of the semigroup may be very small.

\subsection{Useful lemmas.}

We conclude this section by recalling some lemmas, which is often used in the sequel.

Lemma 2.15 (Constancy). Let $K$ be a compact set in $\mathbf{R}^{N}$ and let $h$ be a real valued uppersemicontinuous function on $K$. Let $\phi$ be a $C^{2}$ function on 
$\mathbf{R}^{d}$ with $1 \leq d<N$. Let $G$ be a bounded domain in $\mathbf{R}^{d}$. For each $\zeta \in G$ we assume that there is a maximizer $\left(r_{\zeta}, \rho_{\zeta}\right) \in K$ of

$$
H_{\zeta}(r, \rho)=h(r, \rho)-\phi(r-\zeta)
$$

over $K$ such that $\nabla \phi\left(r_{\zeta}-\zeta\right)=0$. Then,

$$
h_{\phi}(\zeta)=\sup \left\{H_{\zeta}(r, \rho) ;(r, \rho) \in K\right\}
$$

is constant on $G$.

This is elementary and its proof is found in [GG3, Lemma 7.5 and its remark].

Lemma 2.16 (Convergence of maximum points). Let $\left\{U_{\varepsilon}\right\}_{\varepsilon}>0$ be a family of uppersemicontinuous functions on a metric space $X$ with values in $\mathbf{R} \cup\{-\infty\}$. Let $B$ and $S$ be compact sets in $X$ that satisfies $S \subset$ int $B$ (the interior of $B$ ). Assume that $\bar{U}=\limsup _{\varepsilon \rightarrow 0}^{*} U_{e}$ equals a constant $M$ on $S$ and that $\bar{U}(z)<M$ for $z \in B \backslash S$. Let $S_{\varepsilon}$ be the set of maximum points of $U_{e}$ on $B$ i.e.,

$$
S_{\varepsilon}=\left\{z \in B ; U_{\epsilon}(z)=\max _{B} U_{\epsilon}\right\} .
$$

Then there exists a subsequence $\varepsilon^{\prime} \rightarrow 0$ that satisfies

$$
\operatorname{Limsup}_{\varepsilon^{\prime} \rightarrow 0} S_{e^{\prime}} \subset S
$$

in the sense that for each $r>0$ there exists $\varepsilon_{0}$ satisfying

$$
S_{e^{\prime}} \subset\{z \in B ; d(z, S) \leq r\} \quad \text { for all } \varepsilon^{\prime}<\varepsilon_{0}
$$

where $d$ is the distance in $X$. If, moreover, $S$ consists of only one point $\hat{z}$ so that $\bar{U}$ takes a strict maximum at $\hat{z}$ over $B$, then $z_{\varepsilon^{\prime}}$ converges $\hat{z}$. If $U_{\epsilon}$ converges to $\bar{U}$ locally uniformly in a neighborhood of $S$, taking a subsequence is unnecessary. (See Appendix for definition of Limsup.)

This is a slight generalization of [Ba, lemme 4.2, lemme 2.2], where $S$ is assumed to be a singleton.

The next lemma on admissible functions is found in [GG3, Lemma 6.11]. 
Lemma 2.17 (Extension). Let $I$ be an open interval in $(0, T)$ and $J$ be a bounded open interval in $\Omega$. Assume that $\varphi \in C(Q)(Q=(0, T) \times \Omega)$ fulfills

$$
\left.\varphi\right|_{I \times J} \in A_{P}(I \times J)
$$

Let $I_{1}$ and $J_{1}$ be open intervals such that $\bar{I}_{1} \subset I$ and $\bar{J}_{1} \subset J$. Then there is a function $\psi \in A_{P}(Q)$ such that

$$
\varphi \leq \psi \quad \text { in } Q \text { and } \varphi=\psi \text { in } \bar{I}_{1} \times \bar{J}_{1} \text {. }
$$

Sometimes we would like to test non-admissible function for viscosity solutions. As proved in [GG3] there are several equivalent definitions of solutions. The next lemma easily follows from the infinitesimal version of definition [GG3, §6].

Lemma 2.18 (Test functions). Assume that $F \in \mathcal{F}$ and that $W \in \mathcal{E}$ with singularity $P$ of $W$. Assume that $u$ is a subsolution of $(E)$ in $Q=(0, T) \times \Omega$. For $(\hat{t}, \hat{x}) \in Q$ let $I$ and $J$ be open intervals with $\hat{t} \in I \subset(0, T), \hat{x} \in J \subset \Omega$ and let $Q^{\prime}$ denote $Q^{\prime}=I \times J$. Let $\psi \in C(Q)$ be of form

$$
\psi(t, \boldsymbol{x})=g(t)+f(\boldsymbol{x}), \quad g \in C^{1}(I), f \in C(J) .
$$

Assume that $(\psi,(\hat{t}, \hat{x}))$ fulfills

$$
\max _{Q^{\prime}}\left(u^{*}-\psi\right)=\left(u^{*}-\psi\right)(\hat{t}, \hat{x})
$$

and that $f$ is either $P$-faceted at $\hat{x}$ in $J$ with $\hat{x} \in$ int $R(f, \hat{x})$ or $f$ is $C^{2}$ at $\hat{x}$ with $f^{\prime}(\hat{\boldsymbol{x}}) \notin P$. Then (2.7) is still valid.

\section{§3. Sufficient condition for solutions.}

Lemma 3.1. Assume that $F \in \mathcal{F}$ and $W \in \mathcal{E}$ with singularity $P$. Let $u$ be a real valued function on $(0, T) \times \Omega$ that satisfies $u^{*}(z)<\infty$ for all $z \in[0, T) \times \bar{\Omega}$, where $\Omega$ is an open interval. If (2.7) holds for all $(\psi,(\hat{t}, \hat{x})) \in A_{P}(Q) \times Q$ that satisfies (2.8) and one of the following three conditions with $\psi(\hat{t}, x)=f(x)$, then $u$ is a subsolution of $(E)$.

(i) $f^{\prime}(\hat{z}) \notin P$. 
(ii) $f^{\prime}(\hat{x}) \in P$ with $\chi(f, \hat{x})=-1$ and $\hat{x} \in \operatorname{int} R(f, \hat{x})$; moreover,

$$
\left(u^{*}-\psi\right)(\hat{t}, y)<\max _{Q}\left(u^{*}-\psi\right) \quad \text { for } y \in \partial R(f, \hat{x})
$$

(iii) $f^{\prime}(\hat{x}) \in P$ with $\chi(f, \hat{x})=1$ and

$$
u^{*}(\hat{t}, x)=f(\hat{x})+g(\hat{t}) \quad \text { for all } \boldsymbol{x} \in R(f, \hat{\boldsymbol{x}})
$$

Proof. Hexe we set

$$
g(t)=\psi(t, x)-f(x) \quad \text { for } t \in(0, T)
$$

We may assume that $u$ is uppersemicountinuous and $(u-\psi)(\hat{t}, \hat{x})=0$. We must prove (2.7) for all $(\psi,(\hat{t}, \hat{x}))$ (with (2.8)) that satisfies none of conditions (i), (ii), (iii). We may also assume that $f^{\prime}(\hat{x})=0$ by adding some linear function (cf. [GG3,Proposition 2.7]).

Case $1\left(f^{\prime}(\hat{x}) \in P\right.$ and $\left.\chi(f, \hat{x})=0\right)$. It suffices to prove

$$
g^{\prime}(\hat{t})+F(\hat{t}, 0,0) \leq 0
$$

We may assume that $\chi_{-}(f, \hat{x})=-1$ and $\chi_{+}(f, \hat{x})=1$, since the other case $\chi_{-}(f, \hat{x})=1$ and $\chi_{+}(f, \hat{\boldsymbol{x}})=-1$ follows by changing $x$ by $-x$. We set

$$
\hat{y}=\inf \{x \in R(f, \hat{x}) ; u(\hat{t}, x)=f(\hat{x})\} \quad(\in R(f, \hat{x})) .
$$

We shall construct a sequence of functions $\psi_{j}$ approximating $\psi$ so that at a maximum point of $u-\psi_{j}$ the derivative $\left(\psi_{j}\right)_{x}$ does not belong to $P$ and the problem is reduced to (i) to get (2.7). Let $\bar{f} \in C^{2}(\mathbf{R})$ be faceted at $\hat{y}$ with slope 0 that satisfies

$$
\begin{cases}R(\bar{f}, \hat{y})=[\hat{y}-l, \hat{y}] \subset \Omega \quad \text { with some } l>0 \\ \chi(\bar{f}, \hat{y})=1, \\ \bar{f} \notin P \quad \text { on } \bar{J} \backslash R(\bar{f}, \hat{y}) \\ \bar{f}=f \quad \text { on some open bounded neighborhood } J(\bar{f}, \hat{y}) \cap R(f, \hat{y}) \\ \bar{f}>f \quad \text { on } \bar{J} \text { outside } R(\bar{f}, \hat{y}),\end{cases}
$$


Of course for sufficiently small $l>0$ such an $\bar{f}$ exists by setting

$$
\bar{f}(\mathfrak{z})= \begin{cases}A(\mathfrak{x}-\hat{y}-l)^{4}+f(\hat{y}), & \boldsymbol{x}<\hat{y}-l, \\ f(\hat{y}), & \hat{y}-l \leq x \leq \hat{y} \\ (\mathfrak{x}-\hat{y})^{4}+h(x), & \hat{y}<\mathfrak{x}\end{cases}
$$

with $h \in C^{2}(\hat{y}, \infty)$ that agrees with $f$ near $\hat{y}$ and $f \leq h$ in $\Omega \cap(\hat{y}, \infty)$, where ' $A>0$ is taken sufficiently large. We then set

$$
f_{\eta}(x)=\bar{f}(x+\eta) \quad \boldsymbol{x} \in \Omega \quad \text { for } \eta \in \mathbf{R} .
$$

We may assume that $J$ is taken small so that

$$
\begin{aligned}
& f_{\eta}^{\prime} \notin P \quad \text { on } \bar{J} \backslash R\left(f_{\eta}, \hat{y}-\eta\right), \\
& R\left(f_{\eta}, \hat{y}-\eta\right) \subset J
\end{aligned}
$$

for all sufficiently small $\eta$, say $|\eta| \leq \eta_{0}$. Clearly, $\left.f_{\eta}\right|_{J} \in C_{P}^{2}(J)$.

We set

$$
\begin{aligned}
\psi_{\eta}(t, x)=f_{\eta}(x)+\tilde{g}(t) & \text { for }(t, x) \in Q \text { and }|\eta| \leq \eta_{0} \\
\tilde{g}(t)=g(t)+(t-\hat{t})^{2} & \text { for } t \in(0, T)
\end{aligned}
$$

Evidently, $\psi_{\eta}$ converges to $\psi_{0}$ uniformly in $(0, T) \times \bar{J}$ as $\eta \rightarrow 0$. Since $u-\psi_{0}$ takes its strict maximum at $(\hat{t}, \hat{y})$, Lemma 2.16 on convergence of maximum points implies that $\left(t_{\eta}, x_{\eta}\right)$ with

$$
\max _{Q}\left(u-\psi_{\eta}\right)=\left(u-\psi_{\eta}\right)\left(t_{\eta}, x_{\eta}\right)
$$

converges to $(\hat{t}, \hat{y})$ as $\eta \rightarrow 0$. So $\left(t_{\eta}, x_{\eta}\right) \in I \times J$ for all sufficiently small $|\eta|$, say $|\eta|<\eta_{1}\left(<\eta_{0}\right)$, where $I$ is a small open interval in $(0, T)$ containing $\hat{t}$.

(a) We consider the case that $f_{\eta}^{\prime}\left(x_{\eta}\right)=0$ for all sufficiently small $\eta$, say $|\eta| \leq \eta_{2}\left(<\eta_{1}\right)$. Constancy lemma 2.15 implies that

$$
\left(u-\psi_{\eta}\right)\left(t_{\eta}, x_{\eta}\right)=\left(u-\psi_{0}\right)(\hat{t}, \hat{y})=0
$$

for all $|\eta| \leq \eta_{2}$. Since $0=f_{\eta}^{\prime}\left(x_{\eta}\right)$ implies $x_{\eta}+\eta \in R(\bar{f}, \hat{y})$, it follows that $\bar{f}\left(x_{\eta}+\eta\right)=\bar{f}(\hat{y})$. Thus,

$$
u\left(t_{\eta}, x_{\eta}\right)-\tilde{g}\left(t_{\eta}\right)=u(\hat{t}, \hat{y})-\tilde{g}(\hat{t}), \quad|\eta| \leq \eta_{2}
$$


Since $x_{\eta}+\eta \in R(\bar{f}, \hat{y})$ we have $x_{\eta}+\eta \leq \hat{y}$, so that $x_{\eta}<\hat{y}$ for $0<\eta<\eta_{2}$. The strict maximality of $u-\psi_{0}$ at $(\hat{t}, \hat{y})$ yields

$$
u\left(t_{\eta}, x_{\eta}\right)-\bar{f}\left(x_{\eta}\right)-\tilde{g}\left(t_{\eta}\right)<u(\hat{t}, \hat{y})-\bar{f}(\hat{y})-\tilde{g}(\hat{t}) \quad \text { for } 0<\eta<\eta_{2} .
$$

We thus observe that $\bar{f}(\hat{y})<\bar{f}\left(x_{\eta}\right)$ for $0<\eta \leq \eta_{2}$. This implies $x_{\eta}>\hat{y}$ or $x_{\eta}<\hat{y}-l$. Since $x_{\eta}+\eta \in R(\bar{f}, \hat{y}), x_{\eta}<\hat{y}$ for small $\eta>0$. Thus $x_{\eta}<\hat{y}-l$ for small $\eta>0$. This contradicts $x_{\eta} \rightarrow \hat{y}$ as $\eta \rightarrow 0$.

(b) We may now assume that there exists a sequence $\left\{\eta_{j}\right\} \subset(-1,1)$ with $\eta_{j} \rightarrow 0$ as $j \rightarrow \infty$ and $f_{\eta_{j}}^{\prime}\left(\boldsymbol{x}_{\eta_{j}}\right) \neq 0$ for all $j \geq 1$. We shall simply denote $x_{\eta_{j}}$ by $x_{j}$ and $f_{\eta_{j}}$ by $f_{j}$. Since $f_{j}^{\prime}\left(x_{j}\right) \neq 0, x_{j}+\eta_{j} \notin[\hat{y}-l, \hat{y}]=R(\bar{f}, \hat{y})$. Since $x_{j} \rightarrow \hat{y}$ as $j \rightarrow \infty$, we now observe that for sufficiently large $j$,

$$
\boldsymbol{x}_{j}+\eta_{j} \in\{\boldsymbol{x}>\hat{\boldsymbol{y}} ; \boldsymbol{x} \in J\}
$$

with $f_{j}^{\prime}\left(x_{j}\right)=\vec{f}\left(x_{j}+\eta_{j}\right) \notin P$. By Extension lemma 2.17 there exists $\tilde{\psi}_{j} \in A_{P}(Q)$ with $\tilde{\psi}_{j}=\psi_{j}$ on $I \times J$ and $\tilde{\psi}_{j} \geq \psi_{j}$ in $Q$ by taking $I$ and $J$ a little bit smaller. We apply (i) to get

$$
\tilde{g}^{\prime}\left(t_{\eta_{j}}\right)+F\left(t_{\eta_{j}}, f_{j}^{\prime}\left(x_{j}\right), \Lambda_{W}\left(f_{j}, x_{j}\right)\right) \leq 0 .
$$

Since $f_{j}^{\prime}\left(x_{j}\right)$ and $f_{j}^{\prime \prime}\left(x_{j}\right) \rightarrow 0$ as $j \rightarrow \infty$ and since we have assumed that $W^{\prime \prime}(p)$ is bounded around $p=0$, sending $j \rightarrow \infty$ now yields

$$
g^{\prime}(\hat{t})+F(\hat{t}, 0,0) \leq 0
$$

by continuity (F1) of $F$. Thus (2.7) follows for Case 1 .

Case $2\left(f^{\prime}(\hat{x}) \in P\right.$ and $\left.\chi(f, \hat{x})=1\right)$. By (iii) we may assume that there is $x_{0} \in R(f, \hat{x})$ that satisfies $u\left(\hat{t}, x_{0}\right)<f(\hat{x})+g(\hat{t})=u(\hat{t}, \hat{x})$. We may also assume $g(\hat{t})=0$. The idea of the proof is to construct a test fuction $\tilde{\psi}$ so that $\chi(\tilde{\psi}(\hat{t}, \cdot), \hat{x})=0$ by modifying $\psi$, and to reduce the problem to Case 1 . We set $M=\left(u\left(\hat{t}, x_{0}\right)+f(\hat{x})\right) / 2$ and take a rectangular open neighborhood $I_{0} \times J_{0}$ of $\left(\hat{t}, x_{0}\right)$ in $Q$ such that

$$
u(t, x)-g(t)<M \quad \text { for all }(t, x) \in I_{0} \times J_{0} .
$$

Take $\xi \in C^{2}(\Omega)$ that satisfies $\xi=0$ in $\Omega \backslash J_{0}$ and

$$
-\{f(\hat{x})-M\}<\xi(x)<0 \quad \text { for } x \in J_{0} .
$$


Then $\bar{f}=f+\xi$ is faceted at $\hat{\boldsymbol{x}}$ in $\Omega$ with $\chi(\bar{f}, \hat{\boldsymbol{x}})=0$, since $\hat{\boldsymbol{z}} \notin J_{0}$. Since $f \in$ $C_{P}^{2}(\Omega)$ there is an open interval $J \subset \Omega$ containing $R(\bar{f}, \hat{\mathfrak{x}})$ with $\left.\bar{f}\right|_{J} \in C_{P}^{2}(J)$ and $\bar{f}^{\prime}(x) \notin P$ for $\bar{J} \backslash R(\bar{f}, \hat{x})$. We take $B$ large so that

$$
B>\{f(\hat{x})-M\} /(t-\hat{t})^{2} \quad \text { for } t \in(0, T) \backslash I_{0}
$$

and set

$$
\begin{aligned}
& \bar{g}(t)=g(t)+B(t-\hat{t})^{2}, \quad \text { for } t \in(0, T), \\
& \bar{\psi}(t, x)=\bar{f}(x)+\bar{g}(t) \quad \text { for }(t, x) \in Q
\end{aligned}
$$

By this choice of $\bar{\psi}$ we have

$$
\max _{Q}(u-\bar{\psi})(t, x) \leq(u-\bar{\psi})(\hat{t}, \hat{x})=0
$$

Indeed, for $(t, x) \in I_{0} \times J_{0}$

$$
u(t, x)-\bar{\psi}(t, x)<M-f(x)-\xi(x)<0 .
$$

For $x \in J_{0}$ and $t \in(0, T) \backslash I_{0}$

$$
\begin{aligned}
& u(t, x)-\bar{\psi}(t, x)=u(t, x)-f(x)-\xi(x)-\bar{g}(t) \\
& <u(t, x)-f(x)-\xi(x)-g(t)-\{f(\hat{x})-M\} \\
& \leq-\xi(x)-\{f(\hat{x})-M\}<0,
\end{aligned}
$$

since $\bar{g}(t)>g(t)+\{f(\hat{x})-M\}$. For $x \in \Omega \backslash J_{0}$ and $t \in(0, T)$, it is clear that $(u-\bar{\psi})(t, x) \leq(u-\psi)(t, x) \leq 0$.

By definition of $\bar{\psi}$ we see

$$
\left.\bar{\psi}\right|_{I \times J} \in A_{P}(I \times J) \text { and } \bar{\psi} \in C(Q)
$$

with an open interval $I$ s.t. $\hat{t} \in I \subset(0, T)$. Let $J^{\prime}$ be an open interval that satisfies $R(\bar{f}, \hat{x}) \subset J^{\prime}, \bar{J}^{\prime} \subset J$ and let $I^{\prime}$ be an open interval with $\vec{I}^{\prime} \subset I$ and $\hat{t} \in I^{\prime}$. By Extension lemma 2.17 there is $\tilde{\psi} \in A_{P}(Q)$ that satisfies $\tilde{\psi} \geq \bar{\psi}$ in $Q$ and $\tilde{\psi}=\bar{\psi}$ in $\vec{I} \times \vec{J}$. Since $\tilde{\psi} \geq \bar{\psi}$ in $Q, u-\tilde{\psi}$ takes its maximum over $Q$ at $(\hat{t}, \hat{x})$. By definition of $\bar{f}$ we have

$$
\tilde{\psi}_{\mathfrak{x}}(\hat{t}, \hat{x})=0 \in P \quad \text { and } \quad \chi(\tilde{\psi}(\hat{t}, \cdot), \hat{x})=0
$$


By degenerate ellipticity (F2) and $\Lambda_{W}(f, \hat{x}) \geq 0,(2.7)$ for Case 2 follows from Case 1.

Case $3\left(f^{\prime}(\hat{x}) \in P\right.$ and $\left.\chi(f, \hat{x})=-1\right)$. We consider upper canonical modification $f^{\#, \varepsilon} \in C(\Omega)$ of $f$ for sufficiently small $\varepsilon>0$ as in [GG3, $\left.\S 8.3-8.4\right]$ :

$$
f^{\#, \varepsilon}(x)= \begin{cases}f(x)+\left\{f\left(b_{-}+\varepsilon\right)-f\left(b_{-}\right)\right\} & \text {for } x \in\left(a_{-}, b_{-}\right] \\ f(x+\varepsilon) & \text { for } x \in\left(b_{-}, c_{-}-\varepsilon\right), \\ f(\hat{x}) & \text { for } x \in\left[c_{-}-\varepsilon, c_{+}+\varepsilon\right], \\ f(x-\varepsilon) & \text { for } x \in\left(c_{+}+\varepsilon, b_{+}\right), \\ f(x)+\left\{f\left(b_{+}-\varepsilon\right)-f\left(b_{+}\right)\right\} & \text {for } x \in\left[b_{+}, a_{+}\right)\end{cases}
$$

with $\Omega=\left(a_{-}, a_{+}\right)$and $R(f, \hat{x})=\left[c_{-}, c_{+}\right]$, where let $\Omega_{2}=\left(b_{-}, b_{+}\right)$be an open interval as in [GG3, §8.3] (independent of small $\varepsilon>0$ ), so that $R(f, \hat{\boldsymbol{x}}) \subset$ $\Omega_{2} \subset \Omega$ and $f^{\prime}(x) \notin P$ for all $x \in \Omega_{2} \backslash R(f, \hat{x})$. This family satisfies

$$
\begin{aligned}
& R\left(f^{\#, e}, \hat{x}\right)=\left[c_{-}-\varepsilon, c_{+}+\varepsilon\right] \\
& f \leq f^{\#, \varepsilon} \text { in } \Omega \quad \text { with } f^{\#, \varepsilon}(x)=f(\hat{x}) \text { for } x \in R\left(f^{\#, \varepsilon}, \hat{x}\right), \\
& \text { int } R\left(f^{\#, \varepsilon}, \hat{x}\right) \supset R(f, \hat{x}) \text { and } L\left(f^{\#, \varepsilon}, \hat{x}\right)=L(f, \hat{x})+2 \varepsilon .
\end{aligned}
$$

By this choice $\left.f^{\#, \varepsilon}\right|_{\Omega_{2}} \in C_{P}^{2}\left(\Omega_{2}\right)$. Clearly $u-f^{\#, \varepsilon}-g$ does not attain its maximum over $Q$ on the boundary of $R\left(f^{\#, \varepsilon}, \hat{x}\right)$. Using Extension lemma 2.17 as in Case 2, we may regard $f^{\#, e}+g$ as a test function near $\{\hat{t}\} \times$ $R\left(f^{\#, \varepsilon}, \hat{x}\right)$. By (ii) we have

$$
g^{\prime}(\hat{t})+F\left(\hat{t},\left(f^{\#, e}\right)_{x}(\hat{x}),-\frac{\Delta}{L(f, \hat{x})+2 \varepsilon}\right) \leq 0
$$

with $\Delta=W^{\prime}(+0)-W^{\prime}(-0)$. Since $\left(f^{\#, \varepsilon}\right)_{x}(\hat{x})=0$, sending $\varepsilon \rightarrow 0$ yields (2.7) for Case 3 .

Sometimes to conclude (2.7) we may assume that $W$ is strictly convex provided that $\psi$ is 'convex' in $\boldsymbol{x}$.

Lemma 3.2. Assume that $F \in \mathcal{F}$ and $W \in \mathcal{E}$. For $\lambda>0$ define a strict convex function $W_{\lambda} \in \mathcal{E}$ by

$$
W_{\lambda}(p)=W(p)+\frac{\lambda}{2} p^{2}, \quad p \in \mathbf{R}
$$


Let $\Omega$ be open interval. Let $(\hat{t}, \hat{x}) \in Q=(0, T) \times \Omega$ and

$$
\psi(t, x)=f(x)+g(t) \in A_{P}(Q) .
$$

Assume that $f^{\prime \prime}(\hat{x}) \geq 0$. Then

$$
g^{\prime}(\hat{t})+F\left(\hat{t}, f^{\prime}(\hat{x}), \Lambda_{W}(f, \hat{x})\right) \leq 0
$$

if and only if

$$
g^{\prime}(\hat{t})+F\left(\hat{t}, f^{\prime}(\hat{x}), \Lambda_{W_{\lambda}}(f, \hat{x})\right) \leq 0 \quad \text { for all } \lambda>0
$$

(or at least for some sequence $\lambda=\lambda_{j}>0(j=1,2, \cdots)$ converging to zero). Proof. Since the singularity of $W_{\lambda}$ equals the singularity $P$ of $W$, the set of admissible functions $A_{P}(Q)$ is independent of $\lambda$. In this proof we denote $\Lambda_{W}(f, \hat{x})$ (resp. $\left.\Lambda_{W_{\lambda}}(f, \hat{x})\right)$ simply by $\Lambda_{W}$ (resp. $\Lambda_{W_{\lambda}}$ ). For all $\lambda>0$ a direct computation shows that

$$
\Lambda_{W_{\lambda}}= \begin{cases}\Lambda_{W} & \text { if } f \text { is } P \text {-faceted at } \hat{x} \\ \Lambda_{W}+\lambda f^{\prime \prime}(\hat{x}) & \text { otherwise }\end{cases}
$$

so that

$$
\Lambda_{W} \leq \Lambda_{W_{\lambda}}
$$

If (3.1) holds, inequality $\Lambda_{W} \leq \Lambda_{W_{\lambda}}$ yields (3.2) since $F$ is degenerate elliptic by (F2). The proof of 'only if' part is complete.

Assume now that

$$
g^{\prime}(\hat{t})+F\left(\hat{t}, f^{\prime}(\hat{x}), \Lambda_{W_{\lambda_{j}}}\right) \leq 0, \quad j=1,2, \cdots
$$

for some sequence $\lambda_{j}>0(j=1,2, \cdots)$ converging to zero. Since $F$ is continuous by (F1), sending $j$ to infinity yields (3.1). The proof of 'if' part is complete.

Remark 3.3. There is a direct proof of Lemma 3.1 Case 1 without appealing to the sliding argument based on the Constancy lemma. Instead of translation of $\bar{f}$ we modify $\bar{f}$ near $\hat{y}$ so that

$$
\tilde{f}(x)= \begin{cases}\bar{f}(x), & x \in \Omega \backslash(a, b), \\ q(x-a)+\bar{f}(\hat{y}), & x \in(a, b)\end{cases}
$$


with $q=(f(b)-f(a)) /(b-a), \hat{y}-l<a<\hat{y}<b$. Here $b$ is taken sufficiently close to $\hat{y}$ so that

$$
0<\bar{f}^{\prime}(x)<p_{1} / 2, \quad \vec{f}^{\prime \prime}(x)>0 \quad \text { for } \hat{y}<x<b
$$

where

$$
(0<) p_{1}= \begin{cases}\min \{p \in P ; p>0\} & \text { if } \max P>0, \\ \text { arbitrary positive number } & \text { otherwise (i.e. } \max P=0),\end{cases}
$$

so that $0<q<p_{1} / 2$. We set

$$
\begin{gathered}
\varphi^{\mu}(t, x)=g(t)+f^{\mu}(x), \\
f^{\mu}(x)=\mu \tilde{f}(x)+(1-\mu) \bar{f}(x), \quad 0<\mu<1
\end{gathered}
$$

and observe that $\varphi^{\mu}$ converges to $\varphi^{0}\left(=\psi_{0}\right)$ uniformly as $\mu \rightarrow 0$ and that $0<\left(f^{\mu}\right)^{\prime}(x)<p_{1} / 2$ for $x \in(a, b), \mu>0$. Since a maximizer $\left(t_{\mu}, x_{\mu}\right)$ of $u-\varphi^{\mu}$ (over $Q$ ) converges to $(\hat{t}, \hat{x})$ as $\mu \rightarrow 0$ so that $\left(f^{\mu}\right)^{\prime}\left(x_{\mu}\right) \notin P$ for sufficiently small $\mu>0$, we have (by Lemma 2.17)

$$
g^{\prime}\left(t_{\mu}\right)+F\left(t_{\mu},\left(f^{\mu}\right)^{\prime}\left(x_{\mu}\right), \Lambda_{W}\left(f^{\mu}, x_{\mu}\right)\right) \leq 0 .
$$

Sending $\mu$ to zero yields

$$
g^{\prime}(\hat{t})+F(\hat{t}, 0,0) \leq 0
$$

\section{§4. Wulff functions.}

We shall consider the convex conjugate of $W \in \mathcal{E}$ and its translation and dilation which we call a Wulff function. The advantage of a Wulff function $w$ is that the weighted curvature is constant almost everywhere in the domain of definition. We shall study various properties of Wulf functions and its modifications.

\subsection{Fundamental properties.}

Definition 4.1(Unit Wulff function). For $\alpha \in \mathcal{A}$ with $\alpha \not \equiv 0$ let $\beta$ denote

$$
\beta(p)=\int_{+0}^{p} \alpha(q) d q+\left\{\begin{array}{lll}
0 & \text { if } & 0 \notin P \\
\Delta(0) / 2 & \text { if } & 0 \in P
\end{array}\right.
$$


where $P$ denotes the singularity of $W \in \mathcal{E}$ that satisfies $\alpha=W^{\prime \prime}$ and $\Delta(0)=$ $W^{\prime}(+0)-W^{\prime}(-0)$. By definition $\beta(+0)=\Delta(0) / 2, \beta(-0)=-\Delta(0) / 2$ if $0 \in P$. Evidently, $\beta$ is nondecreasing. There is a unique maximal monotone operator $B$ on $\mathbf{R}$ (see Appendix) containing a function $\beta$. We often identify $B$ with $\beta$. Let $\beta^{-1}$ be the inverse operator of $\beta$. By definition the range of $\beta^{-1}$ equals R. We set

$$
-\infty \leq \beta_{-}:=\lim _{p \rightarrow-\infty} \beta(p) \leq \beta_{+}:=\lim _{p \rightarrow+\infty} \beta(p) \leq \infty .
$$

By definition $\beta_{-} \leq 0 \leq \beta_{+}$. From $\alpha \neq 0$ it follows that $\beta_{-}<\beta_{+}$so that the domain of definition of $\beta^{-1}$ is nonempty. We say

$$
\stackrel{\circ}{w}(x)=\int_{0}^{x} \beta^{-1}(q) d q, \quad x \in\left(\beta_{-}, \beta_{+}\right)
$$

is the unit Wulff function (of $\alpha$ ). By definition $\stackrel{\circ}{w}$ is a single-valued convex function on $\left(\beta_{-}, \beta_{+}\right)$. So $\stackrel{\circ}{w}$ is $C^{1}$ except countably many points in $\left(\beta_{-}, \beta_{+}\right)$ and $\stackrel{\circ}{w}^{\prime}$ is nondecreasing, so that $\stackrel{\circ}{w}^{\prime}(x+0), \stackrel{\circ}{w}^{\prime}(x-0)$ is defined for all $x \in$ $\left(\beta_{-}, \beta_{+}\right)$.

It is easy to see that the unit Wulff function $\stackrel{\circ}{w}$ is the restriction of the convex conjugate $W_{C}$ of $W$ on $\left(\beta_{-}, \beta_{+}\right)$, where $W(p)=\int_{0}^{p} \beta(q) d q$ and $W_{C}(q)=$ $\sup _{p}(q p-W(p))$. This $W$ satisfies $W^{\prime}(+0)=-W^{\prime}(-0), W(0)=0, W \geq 0$ in addition to $W^{\prime \prime}=\alpha$.

Let $\Sigma(\stackrel{\circ}{w})$ denote the closed set of points of $\left(\beta_{-}, \beta_{+}\right)$where $\stackrel{\circ}{w}$ is not $C^{2}$. This set may not be discrete in general. By the definition of $\mathcal{A}, \Sigma(\stackrel{\circ}{w})$ consists of critical values of $\beta$ and $\beta(p \pm 0)$ for $p \in P$, i.e.,

$$
\Sigma(\stackrel{\circ}{w})=\left\{\beta(p) ; \beta^{\prime}(p)=0 \text { for some } \quad p \in \mathbf{R} \backslash P\right\} \cup\{\beta(p \pm 0), p \in P\} .
$$

By the definition of $\stackrel{0}{w}$ we observe that for $\hat{x} \in \Sigma(\stackrel{\circ}{w}), \alpha(p)=W^{\prime \prime}(p)=0$ holds for all $p \in\left(\stackrel{\circ}{w}^{\prime}(\hat{\boldsymbol{x}}-0), \stackrel{\circ}{w}^{\prime}(\hat{\boldsymbol{x}}+0)\right)$. Moreover, if $\stackrel{\circ}{w}^{\prime}(\hat{\mathfrak{x}}-0) \notin P$ (resp. $\left.\stackrel{\circ}{w}^{\prime}(\hat{\mathfrak{z}}+0) \notin P\right)$ then $W^{\prime \prime}\left(\stackrel{\circ}{w}^{\prime}(\hat{\boldsymbol{x}}-0)\right)=0\left(\right.$ resp. $\left.W^{\prime \prime}\left(\stackrel{\circ}{w}^{\prime}(\hat{\mathfrak{x}}+0)\right)=0\right)$.

Here the boundedness of $\alpha$ on $K \backslash P$ for a compact set $K$ is invoked to prove that $\beta(p \pm 0)(p \in P)$ actually belongs to $\Sigma(\stackrel{0}{w})$. If $\alpha$ is strictly positive on $\mathbf{R} \backslash P$, then $\stackrel{\circ}{w} \in C^{1}\left(\beta_{-}, \beta_{+}\right)$with

$$
\Sigma(\stackrel{\circ}{w})=\{\beta(p \pm 0) ; p \in P\}
$$


since there is no critical point of $\beta$. We say that $\alpha$ is coercive if $\beta_{ \pm}= \pm \infty$. For example if there is $\lambda>0$ that satisfies $\alpha(p) \geq \lambda$ for all $p \in \mathbf{R} \backslash P$, i.e., $\alpha$ is uniformly positive in $\mathbf{R} \backslash P$, then $\alpha$ is coercive.

Example 4.2. If $W(p)=\lambda p^{2} / 2$ for $\lambda>0$, then $\alpha=W^{\prime \prime}=\lambda$. The unit Wulff function is

$$
\stackrel{\circ}{w}(x)=\frac{x^{2}}{2 \lambda} \quad \text { with } \quad \beta_{ \pm}= \pm \infty
$$

If $W(p)=\left(1+p^{2}\right)^{1 / 2}$ then $\alpha=\left(1+p^{2}\right)^{-3 / 2}$ and $\beta=p\left(1+p^{2}\right)^{-1 / 2}$. The unit Wulff function is

$$
\stackrel{\circ}{w}(x)=-\sqrt{1-x^{2}}+1 \text { with } \beta_{ \pm}= \pm 1 .
$$

In both examples it is easy to see that

$$
\Lambda_{W}(\stackrel{\circ}{w}, \boldsymbol{x})=1, x \in\left(\beta_{-}, \beta_{+}\right) .
$$

(For general $W$ see Proposition 4.4). For smooth interfacial energy Wulff shape has a constant weighted curvature $[\mathrm{Gu}]$. This is why we call $\stackrel{\circ}{w}$ a unit Wulff function. In the second example, $\Lambda_{W}(\stackrel{\circ}{w})$ equals the curvature of the graph of $\stackrel{\circ}{w}$ and the graph of $\stackrel{\circ}{w}$ is in fact a part of circle. If $W(p)=|p|$ so that $W^{\prime \prime}=2 \delta$, then

$$
\stackrel{\circ}{w}(x)=0 \quad \text { for } x \in\left(\beta_{-}, \beta_{+}\right)
$$

with $\beta_{ \pm}= \pm 1$, while

$$
W_{C}(x)= \begin{cases}0 & |x| \leq 1 \\ \infty & \text { otherwise. }\end{cases}
$$

We next rescale and translate the unit Wulff function.

Definition 4.3. Let $\mu$ be nonzero and let $\tilde{x}$ and $\tilde{y}$ be real numbers. For $\alpha \in \mathcal{A}$ with $\alpha \neq \equiv 0$ define $w$ by

$$
w(x ; \mu, \tilde{x}, \tilde{y})=\mu^{-1} \stackrel{\circ}{w}(\mu(x-\tilde{x}))+\tilde{y}
$$

for $x \in\left(a_{-}, a_{+}\right)$with

$$
a_{ \pm}=a_{ \pm}(w)= \begin{cases}\tilde{x}+\beta_{ \pm} / \mu & \text { if } \mu>0 \\ \tilde{x}+\beta_{\mp} / \mu & \text { if } \mu<0\end{cases}
$$


We say that $w(x)=w(x ; \mu, \tilde{x}, \tilde{y}$ ) is the Wulff function (of $\alpha$ ) with weighted curvature $\mu$ and top $(\tilde{x}, \tilde{y})$. This terminology will be justified since $\Lambda_{W}(w)=$ $\mu$ as stated in the next Proposition.

We shall list several elementary properties. As before let $\Sigma(w)$ denote the set of points of $\left(a_{-}, a_{+}\right)$where $w$ is not $C^{2}$. By the definition of $w$ the set $\Sigma(w)$ is of the form

$$
\Sigma(w)=\left\{x \in\left(a_{-}, a_{+}\right) ; \mu(x-\tilde{x}) \in \Sigma(\stackrel{\circ}{w})\right\}
$$

Proposition 4.4. Let $w$ be the Wulf function of $\alpha=W^{\prime \prime} \in \mathcal{A}$ with weighted curvature $\mu \neq 0$ and top $(\tilde{x}, \tilde{y})$. Let $P$ be the singularity of $W$.

(i) $w$ is convex (resp. concave) for $\mu>0$ (resp. $\mu<0$ ).

(ii) $\Sigma(w)=\left\{\tilde{x}+\beta(p) / \mu, \beta^{\prime}(p)=0\right.$ for some $\left.p \in \mathbf{R} \backslash P\right\}$ $\cup\{\tilde{x}+\beta(p \pm 0) / \mu ; p \in P\}$.

For $\hat{x} \in \Sigma(w), \alpha(p)=W^{\prime \prime}(p)=0$ for all $p \in\left(w^{\prime}(\hat{x}-0), w^{\prime}(\hat{x}-0)\right)$. If $w^{\prime}(\hat{\boldsymbol{x}}-0) \notin P\left(\right.$ resp. $\left.w^{\prime}(\hat{\boldsymbol{x}}+0) \notin P\right)$ then $W^{\prime \prime}\left(w^{\prime}(\hat{\boldsymbol{x}}-0)\right)=0$ (resp. $W^{\prime \prime}\left(w^{\prime}(\hat{\boldsymbol{x}}+\right.$ $0))=0$ ).

(iii) If $\alpha$ is positive (i.e. $\alpha(p)>0$ for all $p \in \mathbf{R} \backslash P$ ) and coercive so that $a_{ \pm}= \pm \infty$, then $w \in C^{1}(\mathbf{R})$ and

$$
\Sigma(w)=\{\tilde{x}+\beta(p \pm 0) / \mu ; p \in P\}
$$

(iv) Let $x$ be a point in $\left(a_{-}, a_{+}\right) \backslash \Sigma(w)$. (We futher assume that $w$ is $P$ faceted at $x$ in $\left(a_{-}, a_{+}\right)$so that $\Lambda_{W}(w, x)$ is defined, in other words, $R(w, x) \subset$ $\left(a_{-}, a_{+}\right)$, when $w^{\prime}(x) \in P$.) Then $\Lambda_{W}(w, x)=\mu$.

Proof. Part (i) is trivial. Part (ii) and (iii) follow from the corresponding properties for $\stackrel{\circ}{w}$. We shall prove (iv). By definition

$$
w^{\prime}(x)=\stackrel{\circ}{w}^{\prime}(\mu(x-\tilde{x}))=\beta^{-1}(\mu(x-\tilde{x}))
$$

If $p=w^{\prime}(x) \notin P$ then $\beta\left(w^{\prime}(x)\right)=\mu(x-\tilde{x})$ so

$$
\Lambda_{W}(w, x)=\left(W^{\prime}\left(w^{\prime}(x)\right)\right)_{x}=\left(\beta\left(w^{\prime}(x)\right)+\text { const. }\right)_{x}=\mu
$$


If $p \in P$, then

$$
L(w, x)=(\beta(p+0)-\beta(p-0)) /|\mu|=\Delta(p) /|\mu| .
$$

This yields

$$
\Lambda_{W}(w, x)=\operatorname{sgn} \mu \Delta(p) / L(w, x)=\mu .
$$

\subsection{Wulff functions as test functions.}

We shall show that Wulff functions play a role of test functions, so that these theorems are applied to prove General stability theorem 2.9 in $\S 6$.

Theorem 4.5. Let $w(x)=w(x ; \mu, \tilde{x}, \tilde{y})$ be the Wulff function of $\alpha=W^{\prime \prime} \in$ $\mathcal{A}$ with weighted curvature $\mu \neq 0$ and top $(\tilde{\boldsymbol{x}}, \tilde{y}) \in \mathbf{R}^{2}$. Assume that $\alpha$ is positive in $\mathbf{R} \backslash P$ and coercive and $F \in \mathcal{F}$. Let $\Omega$ be an open interval and let $u$ be a subsolution of $(E)$ in $Q=(0, T) \times \Omega$. Assume that $(\hat{s}, \hat{y}) \in Q$ and

$$
\psi(t, y)=g(t)+w(y) \quad \text { with } \quad g \in C^{1}(0, T)
$$

satisfy

$$
\max _{Q}\left(u^{*}-\psi\right)=\left(u^{*}-\psi\right)(\hat{s}, \hat{y})
$$

and that $R(w, \hat{y}) \subset \Omega$ if $w^{\prime}(\hat{y}) \in P$, where $P$ is the singularity of $W$. Then

$$
g^{\prime}(\hat{s})+F\left(\hat{s}, w^{\prime}(\hat{y}), \mu\right) \leq 0
$$

Proof. Since $\alpha$ is positive in $\mathbf{R} \backslash P$ and coercive, $w \in C^{1}(\mathbf{R})$ and

$$
\Sigma(w)=\{\tilde{x}+\beta(p \pm 0) / \mu ; p \in P\}
$$

by Proposition 4.4, where $\beta$ is the same as in Definition 4.1. If $P$ is empty so that $\Sigma(w)$ is empty, then $w \in C^{2}(\mathbf{R})$ and $\psi \in A_{p}(Q)$ so (4.2) follows from definition of subsolution and Proposition 4.4 (iv).

Now we study general case.

(A) (Case $\hat{y} \notin \Sigma(w)$ ) 
If $w^{\prime}(\hat{y}) \notin P$, then Lemma 2.18 applies $\psi$ at $(\hat{s}, \hat{y})$. So Proposition 4.4 (iv) yields (4.2). On the other hand if $w^{\prime}(\hat{y}) \in P$, then we apply Lemma 2.18 to get (4.2) since $R(w, \hat{y}) \subset \Omega$.

(B) (Case $\hat{y} \in \Sigma(w)$ )

We may assume that $\hat{y} \in \partial R\left(w, y_{0}\right) \subset \Omega$ for some $y_{0} \in \operatorname{int} R\left(w, y_{0}\right)$. We may also assume that $w^{\prime}(\hat{y})=0 \in P$ by adding affine functions to $u$ (cf. [GG3, Proposition 2.7]).

(i) (Case $\mu<0)$. We rescale the graph of $w$ around $\left(y_{0}, w\left(y_{0}\right)\right)$ by

$$
\begin{gathered}
f_{\sigma}(y)=(1-\sigma)^{-1}\left\{w\left((1-\sigma)\left(y-y_{0}\right)+y_{0}\right)-w\left(y_{0}\right)\right\}+w\left(y_{0}\right) \\
\text { for } y \in \mathbf{R} \text { with } 0<\sigma<1
\end{gathered}
$$

and observe that $u^{*}-f_{\sigma}-g$ attains its maximum over $Q$ at $(\hat{s}, \hat{y})$. Since $R\left(w, y_{0}\right) \subset \operatorname{int} R\left(f_{\sigma}, y_{0}\right), \hat{y}$ is now an interior point of $R\left(f_{\sigma}, y_{0}\right)$. Since $u$ is a subsolution, Lemma 2.18 yields

$$
g^{\prime}(\hat{s})+F(\hat{s}, 0,(1-\sigma) \mu) \leq 0 \text { for } \sigma \text { close to zero. }
$$

Indeed, we see that $R\left(f_{\sigma}, y_{0}\right) \subset \Omega$ for $\sigma$ close to zero and Proposition 4.4 (iv) yields

$$
\Lambda_{W}\left(f_{\sigma}, y_{0}\right)=(1-\sigma) \mu
$$

since $f_{\sigma}$ is a Wulff function with curvature $(1-\sigma) \mu$ :

$$
\begin{aligned}
f_{\sigma}(y)= & (1-\sigma)^{-1}\left\{\mu^{-1} \stackrel{\circ}{w}\left(\mu\left((1-\sigma)\left(y-y_{0}\right)+y_{0}-\tilde{x}\right)\right)+\tilde{y}-w\left(y_{0}\right)\right\} \\
& \quad+w\left(y_{0}\right) \\
= & (1-\sigma)^{-1} \mu^{-1} \stackrel{o}{w}((1-\sigma) \mu(y-\bar{x}))+\bar{y} \\
= & w(y ;(1-\sigma) \mu, \bar{x}, \bar{y}) \quad \text { for } \quad y \in \mathbf{R}
\end{aligned}
$$

with

$$
\bar{x}=\left(\tilde{x}-\sigma y_{0}\right) /(1-\sigma) \text { and } \bar{y}=\left(\tilde{y}-\sigma w\left(y_{0}\right)\right) /(1-\sigma) .
$$

Since $0<\sigma<1$ is arbitrary we thus obtain (4.2).

(ii) (Case $\mu>0$ ). We may assume that there is no point $\left(t_{0}, x_{0}\right) \in Q$ satisfying

$$
\left(u^{*}-\psi\right)\left(t_{0}, x_{0}\right)=\left(u^{*}-\psi\right)(\hat{s}, \hat{y}) \quad \text { with } \quad x_{0} \in \operatorname{int} R\left(w, y_{0}\right)
$$


Indeed, if there exists such a point, the problem is reduced to Case (A) since $x_{0} \notin \Sigma(w), w^{\prime}\left(x_{0}\right)=w^{\prime}(\hat{y})$ and $\Lambda_{W}\left(w, x_{0}\right)=\mu$.

We may assume that $\hat{y}$ is the right end point of $R\left(w, y_{0}\right)$. We may also assume that there exists $x_{1} \in \Omega$ such that

$$
\left(u^{*}-\psi\right)(\hat{s}, y)<\left(u^{*}-\psi\right)(\hat{s}, \hat{y})=0 \quad \text { for all } y \in\left(\hat{y}, x_{1}\right) \text { with } \hat{y}<x_{1}
$$

Indeed, if not, there exists a sequence $y_{j} \rightarrow \hat{y}$ as $j \rightarrow \infty$ satisfying ( $u^{*}-$ $\psi)\left(\hat{s}, y_{j}\right)=0, y_{j}>\hat{y}$ and $y_{j} \notin \Sigma(w)$ since $\Sigma(w)$ is a discrete set by assumption of $\alpha$. Applying Case (A) we have

$$
g^{\prime}(\hat{\boldsymbol{s}})+F\left(\hat{\boldsymbol{s}}, w^{\prime}\left(y_{j}\right), \mu\right) \leq 0
$$

by Proposition 4.4 (iv). Sending $y_{j} \rightarrow \hat{y}$, we obtain (4.2) by continuity (F1) of $F$.

We shall show $(4.2)$, when $\left(u^{*}-\psi\right)(\hat{s}, \cdot)$ takes its strict maximum in the interval (inf $R\left(w, y_{0}\right), x_{1}$ ) at $\hat{y}$. We rescale $w$ by

$$
f^{\sigma}(y)=(1+\sigma)^{-1}\{w((1+\sigma)(y-\hat{y})+\hat{y})-\hat{z}\}+\hat{z} \quad \text { for } \quad y \in \mathbf{R}
$$

with $\sigma \geq 0$ and $\hat{z}=w(\hat{y})$. Note that $f^{0}=w$. Since $w^{\prime}$ is monotone nondecreasing, we have $\left(f^{\sigma}\right)^{\prime}(y) \geq w^{\prime}(y)$ for $y>\hat{y}$ and $\left(f^{\sigma}\right)^{\prime}(y) \leq w^{\prime}(y)$ for $y<\hat{y}$, so that

$$
\left\{\begin{array}{lll}
f^{\sigma}>w & \text { on } & \mathbf{R} \backslash R\left(f^{\sigma}, \hat{y}-0\right) \\
f^{\sigma}=w & \text { on } & R\left(f^{\sigma}, \hat{y}-0\right)
\end{array}\right.
$$

for all $\sigma>0$, where

$$
R\left(f^{\sigma}, \hat{y}-0\right):=R\left(f^{\sigma}, \hat{y}-\delta\right)
$$

for sufficiently small $\delta>0$. Setting

$$
\psi^{\sigma}(s, y)=f^{\sigma}(y)+g(s)+(s-\hat{s})^{2}, \quad(s, y) \in(0, T) \times \mathbf{R}
$$

for $\sigma \geq 0$, we see that $u^{*}-\psi^{\sigma}$ takes its strict maximum over $Q$ at $(\hat{s}, \hat{y})$, since $\left(u^{*}-\psi\right)(\hat{s}, \cdot)<0$ on $\operatorname{int} R\left(w, y_{0}\right)$ by our assumption of (ii).

Our aim is to show

$$
g^{\prime}(\hat{s})+F\left(\hat{s}, w^{\prime}(\hat{y}),(1+\sigma) \mu\right) \leq 0
$$


for all small $\sigma>0$. We shall fix small $\sigma>0$ and consider

$$
\varphi_{\rho}^{\sigma}(s, y)=f^{\sigma}(y+\rho)+g(s)+(s-\hat{s})^{2}, \quad(s, y) \in Q
$$

for $\rho \in \mathbf{R},|\rho|<\bar{\rho}$ with small $\bar{\rho}>0$. By Lemma 2.16 on convergence of maximum points if $\left(s_{\rho}, y_{\rho}\right)$ be a point that satisfies

$$
V(\rho):=\max _{Q}\left(u^{*}-\varphi_{\rho}^{\sigma}\right)=\left(u^{*}-\varphi_{\rho}^{\sigma}\right)\left(s_{\rho}, y_{\rho}\right)
$$

then $s_{\rho} \rightarrow \hat{s}$ and $y_{\rho} \rightarrow \hat{y}$ as $\rho \rightarrow 0$.

We shall prove that there is a sequence $\rho_{k} \rightarrow 0$ as $k \rightarrow \infty$ so that $\left(f^{\sigma}\right)^{\prime}\left(y_{\rho_{k}}+\rho_{k}\right) \neq 0$. If not, there exists positive small $\rho^{\circ}<\bar{\rho}$ such that

$$
\left(f^{\sigma}\right)^{\prime}\left(y_{\rho}+\rho\right)=0 \text { for all }|\rho|<\rho^{\circ} .
$$

By Constancy Lemma 2.15, $V(\rho)$ is constant for $|\rho|<\rho^{\circ}$ since $f^{\sigma}$ is $C^{1}$. Thus we see $y_{\rho}+\rho \in R\left(f^{\sigma}, \hat{y}-0\right)$ for $|\rho|<\rho^{0}$ to get $f^{\sigma}\left(y_{\rho}+\rho\right)=w(\hat{y})$. Since $y_{\rho} \rightarrow \hat{y}$ as $\rho \rightarrow 0$ we have $y_{\rho} \in R\left(f^{\sigma}, \hat{y}-0\right)$ for small $\rho>0$, so that $f^{\sigma}\left(y_{\rho}\right)=w(\hat{y})$. From $V(\rho)=V(0)$ for $|\rho|<\rho^{\circ}$, it follows that

$$
\begin{aligned}
& \left(u^{*}-\psi^{\sigma}\right)(\hat{s}, \hat{y})=\left(u^{*}-\varphi_{\rho}^{\sigma}\right)\left(s_{\rho}, y_{\rho}\right) \\
& =\left(u^{*}-\varphi_{0}^{\sigma}\right)\left(s_{\rho}, y_{\rho}\right)=\left(u^{*}-\psi^{\sigma}\right)\left(s_{\rho}, y_{\rho}\right)
\end{aligned}
$$

for small $\rho>0$. Since $u^{*}-\psi^{\sigma}$ takes its strict maximum over $Q$ at $(\hat{x}, \hat{y})$, $y_{\rho^{\prime}}$ should agree with $\hat{y}$. This yields a contradiction to $f^{\sigma}\left(y_{\rho^{\prime}}+\rho^{\prime}\right)=w(\hat{y})$, since $y_{\rho^{\prime}} \leq \hat{y}-\rho^{\prime}<\hat{y}$.

We now have a sequence $\rho_{k} \rightarrow 0$ as $k \rightarrow \infty$ such that $y_{\rho_{k}} \rightarrow \hat{y}, s_{\rho_{k}} \rightarrow \hat{s}$ and $\left(f^{\sigma}\right)^{\prime}\left(y_{\rho}+\rho\right)=w^{\prime}\left((1+\sigma)\left(y_{\rho}+\rho-\hat{y}\right)+\hat{y}\right) \notin P$ for $\rho=\rho_{k}$. This implies $(1+\sigma)\left(y_{\rho}+\rho-\hat{y}\right)+\hat{y} \notin \Sigma(w)$ since $\Sigma(w)=\{\tilde{x}+\beta(p \pm 0) / \mu ; p \in P\}$ as we see in the beginning of the proof. Since $u$ is a subsolution, Lemma 2.18 yields

$$
g^{\prime}\left(s_{\rho}\right)+2\left(s_{\rho}-\hat{s}\right)+F\left(s_{\rho},\left(f^{\sigma}\right)^{\prime}\left(y_{\rho}+\rho\right), \Lambda_{W}\left(f^{\sigma}, y_{\rho}+\rho\right)\right) \leq 0
$$

for $\rho=\rho_{k}$. Sending $\rho_{k} \rightarrow 0$ yields

$$
g^{\prime}(\hat{s})+F\left(\hat{s}, w^{\prime}(\hat{y}),(1+\sigma) \mu\right) \leq 0,
$$

which is what we want to prove. In fact we have $\left(f^{\sigma}\right)^{\prime}(\hat{y})=w^{\prime}(\hat{y})$ and $\Lambda_{W}\left(f^{\sigma}, y_{\rho}+\rho\right)=(1+\sigma) \mu$ from Proposition 4.4 (iv) since $f^{\sigma}$ is a Wulff function: $f^{\sigma}(y)=w(y ;(1+\sigma) \mu,(\tilde{x}+\sigma \hat{y}) /(1+\sigma),(\tilde{y}+\sigma w(\hat{y})) /(1+\sigma))$ for $y \in \mathbf{R}$.

We next generalize this result at least for $\mu<0$ so that it includes the case when $W^{\prime \prime}=\alpha$ is not necessarily positive. 
Theorem 4.6. Let $w(x)=w(x ; \mu, \tilde{x}, \tilde{y})$ be the Wulff function of $\alpha=$ $W^{\prime \prime} \in \mathcal{A}$ with weighted curvature $\mu<0$ and top $(\tilde{x}, \tilde{y})$. Let $\Omega$ be an open interval. Assume that $\bar{\Omega}$ is contained in $\left(a_{-}, a_{+}\right)$, where $a_{ \pm}$is as in Definition 4.3. Let $u$ be a subsolution of $(E)$ with $F \in \mathcal{F}$. Assume that $(\hat{s}, \hat{y}) \in Q=$ $(0, T) \times \Omega$ and

$$
\psi(s, y)=g(s)+w(y) \quad \text { with } \quad g \in C^{1}(0, T)
$$

satisfy (4.1) and that $R(w, \hat{y} \pm 0) \subset \Omega$ if $w^{\prime}(\hat{y} \pm 0) \in P$, where $P$ is the singularity of $W$. Then

$$
g^{\prime}(\hat{s})+\max \left\{F\left(\hat{s}, w^{\prime}(\hat{y}-0), \mu\right), F\left(\hat{s}, w^{\prime}(\hat{y}+0), \mu\right)\right\} \leq 0
$$

Proof.

Step 1. If $\hat{y} \notin \Sigma(w)$, Proposition 4.4 (iv) yields (4.2) as in the proof of Theorem 4.5 (A). If $\hat{y} \in \Sigma(w)$ and $\hat{p}=w^{\prime}(\hat{y}+0) \in P$ we slide $w$ near $\hat{y}$ to get

$$
v_{\eta}(y)= \begin{cases}w(y), & \hat{y} \leq y \\ w(\hat{y})+\hat{p}(y-\hat{y}), & \hat{y}-\eta<y<\hat{y} \\ w(y+\eta), & y \leq \hat{y}-\eta\end{cases}
$$

for sufficiently small $\eta>0$. Since $w$ is concave, $u^{*}-\phi_{\eta}$ with

$$
\phi_{\eta}(s, y)=g(x)+v_{\eta}(y)
$$

takes its maximum over $Q$ still at $(\hat{s}, \hat{y})$. Now $\hat{y}$ is an interior point of $R\left(v_{\eta}, \hat{y}\right) \subset \Omega$ for small $\eta>0$, Lemma 2.18 yields

$$
g^{\prime}(\hat{\boldsymbol{s}})+F(\hat{s}, \hat{p},-\Delta(\hat{p}) /(-\Delta(\hat{p}) / \mu+\eta)) \leq 0
$$

Sending $\eta$ to zero yields

$$
g^{\prime}(\hat{s})+F\left(\hat{s}, w^{\prime}(\hat{y}+0), \mu\right) \leq 0
$$

Step 2. We shall discuss the case $\hat{y} \in \Sigma(w)$ with $w^{\prime}(\hat{y}+0) \notin P$. We rescale $w$ in the form

$$
w_{\sigma}(y)=(1-\sigma)^{-1}\{w((1-\sigma)(y-\hat{y})+\hat{y})-\sigma w(\hat{y})\}
$$


for $0<\sigma<1$ and we shall show that $w-w_{\sigma}$ takes a local strict maximum at $\hat{y}$. In fact we observe that $w_{\sigma}$ is again the Wulff function with weighted curvature $(1-\sigma) \mu$ and top

$$
\left(\frac{\tilde{x}-\sigma \hat{y}}{1-\sigma}, \frac{\tilde{y}-\sigma w(\hat{y} ; \mu, \tilde{x}, \tilde{y})}{1-\sigma}\right)
$$

Since $w^{\prime}(\hat{y}+0) \notin P$

$$
w_{\sigma}(y)>w(y) \text { for } y \in\left(a_{-}, a_{+}\right) \text {with } y>\hat{y}
$$

Indeed, if $y>\hat{y}$, then

$$
y>z:=(1-\sigma)(y-\hat{y})+\hat{y}>\hat{y}
$$

and

$$
w_{\sigma}(y)-w(y)=(1-\sigma)^{-1}\{w(z)-(1-\sigma) w(y)-\sigma w(\hat{y}\} \geq 0
$$

by concavity of $w$. If the equality were valid for some $y>\hat{y}$ in (4.6), then $w$ would be affine in $[\hat{y}, y]$. By definition of $w, w^{i}(y+0)$ would belong to $P$. This contradicts our assumption, so (4.5) follows.

Step 3. Since $w^{\prime}(\hat{y}+0) \notin P$, there is a small open interval $J$ containing $\hat{y}$ that satisfies $\bar{J} \subset \Omega$ and

$$
w^{\prime}(y+0) \notin P \text { for all } y \in J \text { with } y>\hat{y}
$$

By Proposition 4.4 (iv)

$$
\Lambda_{W}\left(w_{\sigma}, y\right)=\mu(1-\sigma), y \in J \backslash \Sigma\left(w_{\sigma}\right), 0<\sigma<1
$$

Step 4. For

$$
\psi_{\sigma}(s, y)=g_{1}(s)+w_{\sigma}(y), 0<\sigma<1
$$

with $g_{1}(s)=g(s)+(s-\hat{s})^{2}$, taking $J$ smaller if necessary, we observe that $u^{*}-\psi_{\sigma}$ takes its maximum over $(0, T) \times J$ at $(\hat{s}, \hat{y})$ and $\left(u^{*}-\psi_{\sigma}\right)(s, y)<\left(u^{*}-\psi_{\sigma}\right)(\hat{s}, \hat{y}),(s, y) \in(0, T) \times J \quad$ with $\quad y>\hat{y} \quad$ or $\quad s \neq \hat{s}$ 
by (4.1) and (4.5) with $w_{\sigma}(\hat{y})=w(\hat{y})$. We then set

$$
\begin{aligned}
& \psi_{\sigma}^{\zeta}(s, y)=g_{1}(s)+w_{\sigma}^{\zeta}(y), \\
& w_{\sigma}^{\zeta}(y)=w_{\sigma}(y+\zeta), \quad \text { with small } \zeta>0 .
\end{aligned}
$$

It turns out that for fixed $\sigma$ there are sequences $\zeta_{j} \downarrow 0,\left(t_{j}, x_{j}\right) \rightarrow(\hat{s}, \hat{y})$ in $(0, T) \times J$ as $j \rightarrow \infty$ that satisfies

$$
\left\{\begin{array}{l}
\max _{(0, T) \times J}\left(u^{*}-\psi_{\sigma}^{\zeta_{j}}\right)=\left(u^{*}-\psi_{\sigma}^{\zeta_{j}}\right)\left(t_{j}, x_{j}\right), \\
x_{j}>\hat{y} \text { and } x_{j} \notin \Sigma\left(w_{\sigma}^{\zeta_{j}}\right) .
\end{array}\right.
$$

The main point is that one can arrange $x_{j} \notin \Sigma\left(w_{\sigma}^{\zeta_{j}}\right)$. We shall postpone the proof in the next subsection $\$ 4.3$ (Corollary 4.11).

Step 5. Since $x_{j} \notin \Sigma\left(w_{\sigma}\right), \psi_{\sigma}^{\zeta_{j}}$ is regarded as a test function in $(0, T) \times J$ near $\left(t_{j}, x_{j}\right)$. Since $u$ is a subsolution, by Lemma 2.18

$$
g_{1}^{\prime}\left(t_{j}\right)+F\left(t_{j},\left(w_{\sigma}^{\zeta_{j}}\right)^{\prime}\left(x_{j}\right), \Lambda_{W}\left(w_{\sigma}^{\zeta_{j}}, x_{j}\right)\right) \leq 0 .
$$

Since $\Lambda_{W}\left(w_{\sigma}^{\zeta}, x_{j}\right)=\mu(1-\sigma)$ and

$$
\left(w_{\sigma}^{\zeta_{j}}\right)^{\prime}\left(x_{j}\right)=w_{\sigma}^{\prime}\left(x_{j}+\zeta_{j}\right) \rightarrow w_{\sigma}^{\prime}(\hat{y}+0)=w^{\prime}(\hat{y}+0) \quad \text { as } \quad j \rightarrow \infty,
$$

sending $j$ to infinity in the preceding inequality yields

$$
g^{\prime}(\hat{s})+F\left(\hat{s}, w^{\prime}(\hat{y}+0), \mu(1-\sigma)\right) \leq 0 .
$$

Since $0<\sigma<1$ is arbitrary, this yields

$$
g^{\prime}(\hat{s})+F\left(\hat{s}, w^{\prime}(\hat{y}+0), \mu\right) \leq 0 .
$$

Analysis symmetric to Steps 1-5 for $w^{\prime}(\hat{y}-0)$ yields

$$
g^{\prime}(\hat{s})+F\left(\hat{s}, w^{\prime}(\hat{y}-0), \mu\right) \leq 0 .
$$

We have thus proved (4.4).

Remark 4.7. If $W \in \mathcal{E}$ is piecewise linear so that the Wulff function is piecewise linear, then $y \in \Sigma(w)$ implies $w^{\prime}(y \pm 0) \in P$. Thus arguments in Steps 2-5 are unnecessary for the proof of Theorem 4.6 in this case. We allow arbitrary degeneracy of $\alpha=W^{\prime \prime}$ that makes the proof technical.

\subsection{Application of Sard's theorem.}

We shall complete the argument in Step 4 of the proof of Theorem 4.6. 
Lemma 4.8. Let $\boldsymbol{x}=\boldsymbol{x}(\zeta)$ be a real-valued nondecreasing function defined on $[0, \eta]$ with $\eta>0$. Let $Z$ denote the set of discontinuities of $x(\zeta)$ in $[0, \eta]$. Let $S_{\eta}$ denote the set of form

$$
S_{\eta}=\{x(\zeta)+\zeta ; 0 \leq \zeta \leq \eta, \zeta \notin Z\}
$$

Then $\left|S_{\eta}\right| \geq \eta$, where $\left|S_{\eta}\right|$ denotes the Lebesgue measure of $S_{\eta}$. In general if

$$
S_{\eta}=\{x(\zeta)+\theta(\zeta) ; 0 \leq \zeta \leq \eta, \zeta \notin Z\}
$$

with a strictly increasing continuous function $\theta$ on $[0, \eta]$, then $\left|S_{\eta}\right| \geq \theta(\eta)-$ $\theta(0)$.

Proof. Since $x(\zeta)+\zeta$ is strictly increasing,

$$
S_{\eta}=[x(0), x(\eta)+\eta] \backslash \bigcup_{\rho \in Z}[x(\rho-0)+\rho, x(\rho+0)+\rho]
$$

and the union is the disjoint union, where we interpret $x(0-0)=x(0)$, $x(\eta+0)=x(\eta)$. Since $Z$ is (at most) countable by monotonicity of $x(\zeta)$,

$$
\left.\left|S_{\eta}\right|=\mid[x(0), x(\eta)+\eta)\right]\left|-\sum_{\rho \in Z}\right|[x(\rho-0)+\rho, x(\rho+0)+\rho] \mid
$$

Noting

$$
|[x(\rho-0)+\rho, x(\rho+0)+\rho]|=|(x(\rho-0), x(\rho+0))| \quad \text { for } \quad \rho \in Z,
$$

yields

$$
\left|S_{\eta}\right|=(x(\eta)+\eta-x(0))-\sum_{\rho \in Z}|(x(\rho-0), x(\rho+0))|
$$

By nondecreasing property of $x$, the last sum is dominated by $x(\eta)-x(0)$. Thus

$$
\left|S_{\eta}\right| \geq(x(\eta)+\eta-x(0))-(x(\eta)-x(0))=\eta
$$

The proof for the general statement is similar so is omitted. 
Lemma 4.9. Let $w$ be the Wulff function of $\alpha=W^{\prime \prime} \in \mathcal{A}$ with weighted curvature $\mu \neq 0$ and top $(\tilde{x}, \tilde{y})$. Then $|\Sigma(w)|=0$, where $|\Sigma(w)|$ denotes the Lebesgue measure of $\Sigma(w)$.

Proof. We may assume that $w$ is the unit Wulf function $\stackrel{\circ}{w}$. Recall that $\Sigma(\stackrel{\circ}{w})$ consists of union of a discrete set $\{\beta(p \pm 0) ; p \in P\}$ and the set of critical values of $\beta$. The first set has the measure zero. The measure of critical values of $\beta \in C^{1}(\mathbf{R} \backslash P)$ is zero by Sard's theorem, so $|\Sigma(\stackrel{\circ}{w})|=0$.

Theorem 4.10. Let $w$ be the Wulff function of $\alpha=W^{\prime \prime} \in \mathcal{A}$ with weighted curvature $\mu<0$ and top $(\tilde{x}, \tilde{y})$. Let $\left(a_{-}, a_{+}\right)$be the domain of definition of $w$. Let $r$ be an uppersemicontinuous function on an interval $\Omega$ with $\bar{\Omega} \subset\left(a_{-}, a_{+}\right)$. Assume that $\hat{\mathfrak{x}} \in \Sigma(w) \cap \Omega$ satisfies

$$
\begin{aligned}
& (r-w)(x) \leq(r-w)(\hat{x}) \quad \text { for all } \quad x \in \Omega, \\
& (r-w)(x)<(r-w)(\hat{x}) \quad \text { for all } \quad x>\hat{x}, \quad(\text { resp. } x<\hat{x}), \quad x \in \Omega
\end{aligned}
$$

and that $w^{\prime}(\hat{x}+0) \notin P$ (resp. $\left.w^{\prime}(\hat{x}-0) \notin P\right)$. Then there are sequences $\zeta_{j} \downarrow 0$ and $x_{j} \downarrow \hat{x}$ (resp. $x_{j} \uparrow \hat{x}$ ) (monotone convergence) that satisfies

$$
\max _{\Omega}\left(r-w^{\zeta_{j}}\right)=\left(r-w^{\zeta_{j}}\right)\left(x_{j}\right) \quad \text { with } \quad x_{j}+\zeta_{j} \notin \Sigma(w) \quad\left(\text { resp. } x_{j}-\zeta_{j} \notin \Sigma(w)\right)
$$

where $w^{\zeta}(x)=w(x+\zeta)($ resp. $=w(x-\zeta))$ for small $\zeta>0$

Proof. We shall discuss the case $w^{\prime}(\hat{x}+0) \notin P$ only since the proof for $w^{\prime}(\hat{x}-0) \notin P$ is similar. Since $w^{\prime}(\hat{x}+0) \notin P$ implies that $w$ has no affine portion in $[\hat{x}, \hat{x}+\rho]$ for small $\rho>0, w$ is strictly concave in $[\hat{x}, \hat{x}+\rho]$.

Since $w$ is concave, for $x<\hat{x}$ and small $\xi>0$

$$
w(x)-w(x+\xi)<w(\hat{x})-w(\hat{x}+\xi)
$$

This implies $x_{\xi} \geq \hat{x}$ for small $\xi>0$ where $x_{\xi}$ be a maximum point of $r-w^{\xi}$ on $\Omega$. Indeed if $x<\hat{x}$, then

$$
\begin{aligned}
\left(r-w^{\xi}\right)(x) & =r(x)-w(x)+w(x)-w(x+\xi) \\
& <r(x)-w(x)+w(\hat{x})-w^{\xi}(\hat{x}) \\
& \leq\left(r-w^{\xi}\right)(\hat{x})
\end{aligned}
$$


so that $x_{\xi} \geq \hat{x}$. (Note that $x_{\xi}$ is well-defined for small $\xi$ since $\bar{\Omega} \subset\left(a_{1}, a_{2}\right)$.)

By strictly concavity of $w$ on $[\hat{x}, \hat{x}+\rho]$, for $x<y, 0 \leq \zeta<\xi$ with $\hat{x} \leq$ $\boldsymbol{x}, \boldsymbol{y}+\boldsymbol{\xi} \leq \hat{\boldsymbol{x}}+\rho$ the inequality

$$
w(x+\zeta)-w(x+\xi)<w(y+\zeta)-w(y+\xi)
$$

or

$$
w^{\zeta}(x)-w^{\xi}(x)<w^{\zeta}(y)-w^{\xi}(y)
$$

is valid. Applying this inequality with $\hat{x} \leq x<y:=x_{\zeta} \leq \hat{x}+\rho-\xi$ and small $\xi>\zeta>0$ yields

$$
\begin{aligned}
\left(r-w^{\xi}\right)(x) & =\left(r-w^{\zeta}\right)(x)+w^{\zeta}(x)-w^{\xi}(x) \\
& <\left(r-w^{\zeta}\right)(x)+w^{\zeta}\left(x_{\zeta}\right)-w^{\xi}\left(x_{\zeta}\right) \\
& \leq\left(r-w^{\xi}\right)\left(x_{\zeta}\right),
\end{aligned}
$$

since $x_{\zeta}$ is a maximum point of $w^{\zeta}$.

Thus we obtain the inequality

$$
\left(r-w^{\xi}\right)(x)<\left(r-w^{\xi}\right)\left(x_{\zeta}\right) \quad \text { for all } x<x_{\zeta}, \quad x \in \Omega
$$

for sufficiently small $\xi>\zeta \geq 0$. By definition of $x_{\zeta}$ we see $x_{\xi} \geq x_{\zeta}$ for small $\xi \geq \zeta \geq 0$. Since the convergence of maximum points yields $x_{\zeta} \rightarrow \hat{x}$ as $\zeta \rightarrow 0$, the function $x(\zeta):=x_{\zeta}$ is nondecreasing in $\zeta \in[0, \eta]$ for sufficiently small $\eta>0$.

Applying Lemma 4.8 to $x(\zeta)=x_{\zeta}$ we observe that

$$
\left|I_{\eta}\right| \geq\left|S_{\eta}\right| \geq \eta>0
$$

where

$$
\begin{aligned}
& S_{\eta}=\left\{x_{\zeta}+\zeta \in I_{\eta}, \zeta \notin Z\right\} \subset I_{\eta}=\left\{x_{\zeta}+\zeta ; 0 \leq \zeta \leq \eta\right\} \\
& Z=\left\{\zeta ; x_{\zeta} \text { is not continuous at } \zeta \in[0, \eta]\right\} .
\end{aligned}
$$

Since $|\Sigma(w)|=0$ by Lemma 4.9, for each (small) $\eta>0$ there exists $\zeta=$ $\zeta(\eta) \in[0, \eta]$ that satisfies $x_{\zeta}+\zeta \notin \Sigma(w)$. Taking $\eta=1 / j(j \geq 1)$ and $\zeta_{j}=\zeta(1 / j)(\leq \zeta(1 /(j-1))$ for $j \geq 2)$ we now obtain desired sequences $\zeta_{j} \downarrow 0$ and $x_{j}:=x_{\zeta_{j}}$ that satisfies

$$
x_{j}+\zeta_{j} \notin \Sigma(w) \text { and } x_{j} \downarrow \hat{x}
$$

since $x_{\zeta}$ is nondecreasing in $\zeta$. 
Corollary 4.11. Assume the same hypotheses of Theorem 4.10 concerning $w$ and $\Omega$. For $g \in C^{1}(0, T)$ let $\psi$ and $\psi^{\zeta}(\zeta>0)$ be

$$
\psi(t, x)=w(x)+g(t), \psi^{\zeta}(t, x)=w^{\zeta}(x)+g(t)
$$

for $(t, x) \in Q=(0, T) \times \Omega$. Let $u$ be a function on $Q$. Assume that $u^{*}-\psi$ takes its maximum $Q$ at $(\hat{s}, \hat{y})$, and

$$
\begin{aligned}
& \left(u^{*}-\psi\right)(t, x)<\left(u^{*}-\psi\right)(\hat{s}, \hat{y}) \\
& \quad \text { for all }(t, x) \in Q \text { with } x>\hat{y}(\text { resp. } x<\hat{y}) \text { or } t \neq \hat{s} \text {. }
\end{aligned}
$$

Assume that $\hat{y} \in \Sigma(w) \cap \Omega$ and that $w^{\prime}(\hat{y}+0) \notin P\left(\right.$ resp. $\left.w^{\prime}(\hat{y}-0) \notin P\right)$. Then there are sequences $\zeta_{j} \downarrow 0, x_{j} \downarrow \hat{y}$ (resp. $x_{j} \uparrow \hat{y}$ ) (monotone convergence), $t_{j} \rightarrow \hat{s}$ that satisfies

$$
\max _{Q}\left(u^{*}-\psi^{\zeta_{j}}\right)=\left(u^{*}-\psi^{\zeta_{j}}\right)\left(t_{j}, x_{j}\right) \quad \text { with } \quad x_{j}+\zeta_{j} \notin \Sigma(w)
$$

where $w^{\zeta}(x)=w(x+\zeta)($ resp. $=w(x-\zeta))$ for small $\zeta>0$.

Proof. We may assume that $w^{\prime}(\hat{y}+0) \notin P$. Let $\left(t_{\zeta}, x_{\zeta}\right)$ denote a maximum of $u^{*}-\psi$ over $Q$. By Lemma 2.16 on convergence of maximum points $t_{\zeta} \rightarrow$ $\hat{\boldsymbol{s}}, x_{\zeta} \rightarrow \hat{y}$ as $\zeta \rightarrow 0$. We argue as in the proof of Theorem 4.10 and observe that $x_{\zeta}$ is nondecreasing in $\zeta \in[0, \eta]$ for sufficiently small $\eta$ and $x_{\zeta} \geq \hat{y}$. For example to see $x_{\zeta} \leq x_{\xi}$ for $0 \leq \zeta \leq \xi$ we calculate

$$
\begin{aligned}
& \left(u^{*}-\psi^{\xi}\right)(t, x)=\left(u^{*}-\psi^{\zeta}\right)(t, x)+w^{\zeta}(x)-w^{\xi}(x) \\
& <\left(u^{*}-\psi^{\zeta}\right)(t, x)+w^{\zeta}\left(x_{\zeta}\right)-w^{\xi}\left(x_{\zeta}\right) \\
& \leq\left(u^{*}-\psi^{\xi}\right)\left(t_{\zeta}, x_{\zeta}\right) \quad \text { for } \quad x<x_{\zeta} \quad \text { with small } \xi>\zeta \geq 0 .
\end{aligned}
$$

As in the proof of Theorem 4.10 we obtain sequences $\zeta_{j} \downarrow 0, x_{j} \downarrow \hat{y}$ and $t_{j} \rightarrow \hat{s}$ such that

$$
\max _{Q}\left(u^{*}-\psi^{\zeta_{j}}\right)=\left(u^{*}-\psi^{\zeta_{j}}\right)\left(t_{j}, x_{j}\right) \quad \text { with } \quad x_{j}+\zeta_{j} \notin \Sigma(w)
$$

The proof is now complete.

\subsection{Cutoff Wulff functions.}


If $\alpha$ is not coercive, the domain $\left(\beta_{-}, \beta_{+}\right)$of the unit Wulff function $\stackrel{\circ}{w}$ is strictly smaller than $\mathbf{R}$. For example if $\varlimsup_{|p| \rightarrow \infty} W(p) /|p|<\infty$, then $\beta_{+}<\infty$ and $\beta_{-}>-\infty$. For later convenience we modify $\stackrel{\circ}{w}(y)$ for large $y$ so that it is defined on whole $\mathbf{R}$.

Definition 4.12. Let $\stackrel{a}{w}$ be the unit Wulff function of $\alpha \in \mathcal{A}$. For $-\infty<$ $q_{-}<0<q_{+}<\infty$ let $\stackrel{\circ}{\rho}$ be of the form

$$
\stackrel{\circ}{\rho}\left(x, q_{-}, q_{+}\right)=\min \left\{\max \left\{\stackrel{\circ}{w}^{\prime}(x), q_{-}\right\}, q_{+}\right\} \quad \text { for } \quad x \in \mathbf{R} \backslash \Sigma(\stackrel{\circ}{w}) \text {. }
$$

Here we interpret $\stackrel{\circ}{w}^{\prime}(x)=+\infty$ (resp. $-\infty$ ) for $x \geq \beta_{+}$(resp. $x \leq \beta_{-}$). For $\boldsymbol{x} \in \mathbf{R}$ let $\stackrel{0}{\tilde{w}}(\boldsymbol{x})=\stackrel{\circ}{w}\left(\boldsymbol{x} ; q_{-}, q_{+}\right)$be defined by

$$
\stackrel{\circ}{\tilde{w}}\left(x ; q_{-}, q_{+}\right)=\int_{0}^{x} \stackrel{\circ}{\rho}\left(z ; q_{-}, q_{+}\right) d z .
$$

(This is well defined since $\beta_{-} \leq 0 \leq \beta_{+}$.) For later convenience we set

$$
\stackrel{\circ}{\tilde{w}}\left(x ; q_{-}, q_{+}\right)= \begin{cases}q_{+} x & x \geq 0 \\ q_{-} x & x \leq 0\end{cases}
$$

when $\alpha \equiv 0$. We say that $\stackrel{\circ}{\tilde{w}}$ is a cutoff unit Wulff function with slopes $\left(q_{-}, q_{+}\right)$.

Let $w$ be the Wulff function with weighted curvature $\mu \neq 0$ and top $(\tilde{x}, \tilde{y})$. Let $\tilde{\boldsymbol{w}}$ be of the form

$$
\tilde{w}\left(x ; \mu, \tilde{x}, \tilde{y}, q_{-}, q_{+}\right)=\mu^{-1} \stackrel{o}{\tilde{w}}\left(\mu(x-\tilde{x}) ; q_{-}, q_{+}\right)+\tilde{y}
$$

We say that $\tilde{w}$ is a cutoff Wulff function (of $\alpha$ ) with weighted curvature $\mu$, slopes $\left(q_{-}, q_{+}\right)$and top $(\tilde{x}, \tilde{y})$.

Let us list a few properties of $\tilde{w}$. By definition

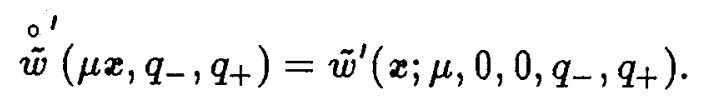

If $\Sigma(\stackrel{\circ}{\tilde{w}})$ is the set of points (in $\mathbf{R}$ ) at which $\stackrel{0}{\tilde{w}}$ is not $C^{2}$, then

$$
\Sigma(\stackrel{\circ}{\tilde{w}})=\Sigma(\stackrel{\circ}{w}) \cup\left\{{\stackrel{\circ}{\gamma_{+}},}_{\gamma_{-}^{\circ}}\right\}
$$


with

$$
\begin{aligned}
& \dot{\gamma}_{+}^{\circ}=\inf \left\{x ; \stackrel{\circ}{w}^{\prime}(x+0) \geq q_{+}\right\}=\inf \left\{x ; \stackrel{\circ}{w}^{\prime}(x+0) \geq q_{+}\right\} \\
& \gamma_{-}^{\circ}=\sup \left\{x ; \stackrel{\circ}{w}^{\prime}(x-0) \leq q_{-}\right\}=\sup \left\{x ; \stackrel{\circ}{w}^{\prime}(x-0) \leq q_{-}\right\}
\end{aligned}
$$

(Note $\stackrel{\circ}{w}$ cannot be $C^{1}$ at the boundary of faceted region of $w$ since we have assumed that $\alpha$ is bounded on every bounded set in $\mathbf{R} \backslash P$.) Evidently the set of points where $\tilde{w}$ is not $C^{2}$ is

$$
\Sigma(\tilde{w})=\Sigma(w) \cup\left\{\gamma_{-}(w), \gamma_{+}(w)\right\}
$$

with

$$
\gamma_{ \pm}= \begin{cases}\gamma_{ \pm}(w)=\tilde{x}+\gamma_{ \pm}^{\circ} / \mu & \text { if } \mu>0 \\ \gamma_{ \pm}(w)=\tilde{x}+\gamma_{\mp}^{\circ} / \mu & \text { if } \mu<0 .\end{cases}
$$

We shall denote $\gamma_{ \pm}$also by $\gamma_{ \pm}(\tilde{w})$. At least on $\left(\gamma_{-}, \gamma_{+}\right), \tilde{w}=w$.

Proposition 4.13. Let $\tilde{w}(x)=\tilde{w}\left(x ; \mu, \tilde{x}, \tilde{y}, q_{-}, q_{+}\right)$be a cutoff Wulff function (of $\alpha=W^{\prime \prime} \in \mathcal{A}$ ) with weighted curvature $\mu \neq 0$, slopes $\left(q_{-}, q_{+}\right)$and top $(\tilde{x}, \tilde{y})$, where $q_{-}<0<q_{+}$. Let $\gamma_{ \pm}(\tilde{w})$ be as in (4.7)-(4.8). Then

$$
\Lambda_{W}(\tilde{w}, x)=\mu \quad \text { for } \quad x \in\left(\gamma_{-}, \gamma_{+}\right) \backslash \Sigma(\tilde{w}) .
$$

Proof. Since $\tilde{w}=w$ with Wulff function $w=w(x ; \mu, \tilde{x}, \tilde{y})$ and $\tilde{w}$ is continuous in $\mathbf{R}$, if $\boldsymbol{w}^{\prime}(\boldsymbol{x}) \in P$ then $\tilde{w}$ is faceted at $\boldsymbol{x}$ in $\mathbf{R}$ with $R(\tilde{w}, \boldsymbol{x}) \subset\left[\gamma_{-}, \gamma_{+}\right]$. As in the proof of Proposition 4.4 (iv) we actually obtain the value of the weighted curvature for all $x \in\left(\gamma_{-}, \gamma_{+}\right) \backslash \Sigma(w)$.

Note that if $\stackrel{\circ}{w}$ is affine on some interval $\left(\beta_{+}-\delta, \beta_{+}\right)$with $\beta_{+}<+\infty$ and $\delta>0$, then $\stackrel{\circ}{w}$ is not faceted at $x \in\left(\beta_{+}-\delta, \beta_{+}\right)$in $\left(\beta_{-}, \beta_{+}\right)$. The weighted curvature of $\stackrel{o}{w}$ at such a point is not defined. This is why we impose extra assumptions on $w$ in Proposition 4.4 (iv). We introduced cutoff Wulff functions so that the weighted curvature is defined for all points we are interested in.

The results in Theorem 4.6 is extended to cutoff Wulff functions. Since the proof is similar we just give its statement, which shall be applied to prove General stability theorem 2.9 . 
Theorem 4.14. Let $\tilde{w}(x)=\tilde{w}\left(x ; \mu, \tilde{x}, \tilde{y}, q_{-}, q_{+}\right)$be a cutoff Wulff function (of $\alpha=W^{\prime \prime} \in \mathcal{A}$ ) with weighted curvature $\mu<0$, slopes $\left(q_{-}, q_{+}\right)$for $q_{ \pm} \gtrless 0$ and top $(\tilde{x}, \tilde{y})$. Let $\gamma_{ \pm}=\gamma_{ \pm}(\tilde{w})$ be as in (4.7) and (4.8). Let $\Omega$ be an open interval. Let $u$ be a subsolution of $(E)$ in $Q=(0, T) \times \Omega$ with $F \in \mathcal{F}$. Assume that $(\hat{s}, \hat{y}) \in Q$ and

$$
\psi(s, y)=g(s)+\tilde{w}(y) \quad \text { with } \quad g \in C^{1}(0, T)
$$

satisfy (4.1). Assume that $\hat{y} \in\left(\gamma_{-}, \gamma_{+}\right)$and that $R(\tilde{w}, \hat{y} \pm 0) \subset \Omega$ if $\tilde{w}^{\prime}(\hat{y} \pm 0) \in$ $P$, where $P$ is singularity of $W$. Then

$$
g^{\prime}(\hat{s})+\max \left\{F\left(\hat{s}, \tilde{w}^{\prime}(\hat{y}-0), \mu\right), F\left(\hat{s}, \tilde{w}^{\prime}(\hat{y}+0), \mu\right)\right\} \leq 0
$$

\section{$\S 5$. Convergence of Wulff functions.}

We study several properties of the Wulff functions $w^{\varepsilon}$ of $\alpha_{\varepsilon}=W_{\varepsilon}^{\prime \prime} \in \mathcal{A}$ when $\alpha_{\varepsilon}$ converges to $\alpha_{0} \in \mathcal{A}$ weakly as measures.

Throughout this section for $\varepsilon \geq 0$ let $w^{\varepsilon}$ be the Wulff function of $\alpha_{\varepsilon}=W_{\varepsilon}^{\prime \prime}$ (with weighted curvature $\mu$ and top $(\tilde{x}, \tilde{y})$ independent of $\varepsilon$ ), where $\alpha_{\varepsilon} \in \mathcal{A}$ with $\alpha_{\varepsilon} \not \equiv 0$ and $\mu \neq 0$. Let $P_{\varepsilon}$ be the singularity of $W_{\varepsilon}$. Let $\tilde{w}^{\varepsilon}$ be the cutoff Wulff function of $\alpha_{e}$ (with weighted curvature $\mu$, top $(\tilde{\boldsymbol{x}}, \tilde{y})$ and slopes $\left(q_{-}, q_{+}\right)$independent of $\varepsilon$, where $\left.q_{-}<0<q_{+}\right)$. For $\mu>0$ we extend $w^{e}(\varepsilon \geq 0)$ as a lowersemicontinuous convex function on $\mathbf{R}$ with values $+\infty$ outside the domain of definition of $w^{e}$ so that its subdifferential (still denoted $\left.\partial w^{e}\right)$ is defined as a maximal monotone operator in Appendix. For $\mu<0$ we interpret the superdifferential in a similar way.

Lemma 5.1. Assume that $\alpha_{e}$ converges to $\alpha_{0}$ as $\varepsilon \rightarrow 0$ weakly as measures.

(i) $w^{\varepsilon}$ converges to $w^{0}$ locally uniformly in the domain of definition $\left(a_{-}\left(w^{0}\right), a_{+}\left(w^{0}\right)\right)$ of $w^{0}$.

(ii) $\partial w^{\varepsilon} \rightarrow \partial w^{0}(\varepsilon \rightarrow 0)$ in the sense of uppersemiconvergence of (maximal monotone) graphs. In other words for any bounded set $K$ in $\mathbf{R}^{2}=\mathbf{R} \times \mathbf{R}$ and any $\eta>0$ there exists $\varepsilon_{1}>0$ such that

$K \cap \operatorname{graph}\left(\partial w^{e}\right) \subset\left\{z=(x, y) \in K ; d\left(z, \operatorname{graph}\left(\partial w^{0}\right)\right)<\eta\right\}$ 
for all $0<\varepsilon<\varepsilon_{1}$, where d denotes the standard distance in $\mathbf{R}^{2}$. Here $\partial w^{\varepsilon}$ denote the subdifferential (resp. superdifferential) of $w^{\epsilon}$ for $\mu>0$ (resp. $\mu<0$ ).

(iii) Let $K$ be a compact set in $\left(a_{-}\left(w^{0}\right), a_{+}\left(w^{0}\right)\right)$ outside jump discontinuity of the derivative $\left(w^{0}\right)^{\prime}$. Then $w^{e}$ converges to $w^{0}$ uniformly with its derivatives in $K$.

(iv) The same assertion (i)-(iii) holds for the cutoff Wulff functions $\tilde{w}^{\varepsilon}$ if we replace $\left(a_{-}\left(w^{0}\right), a_{+}\left(w^{0}\right)\right)$ by $\mathbf{R}$ even if $\alpha_{\varepsilon} \equiv 0$ for some $\varepsilon \geq 0$.

These convergence results are standard in convex analysis (see e.g. [A] where the relation of convergence of convex functions and their subdifferentials and conjugates for general functionals on an infinite dimensional Hilbert space are presented. We give an elementary proof of Lemma 5.1 in the end of Appendix. In the proof of General stability theorem 2.9, the next lemma is important to apply Theorem 4.5 and Theorem 4.6 .

Lemma 5.2. Assume that $\alpha_{e}$ converges to $\alpha_{0}$ weakly as measures as $\varepsilon \rightarrow 0$. Let $I$ be a bounded interval with $\bar{I} \subset\left(a_{-}\left(w^{0}\right), a_{+}\left(w^{0}\right)\right)$. Assume that either (i) $I \cap \Sigma\left(w^{0}\right)=\emptyset$ and $\left(w^{0}\right)^{\prime}(x) \notin P_{0}$ for all $x \in I$ or (ii) $w^{0}$ is $P_{0}$-faceted at some point $x_{0} \in$ int $I$ (so that $R\left(w^{0}, x_{0}\right) \subset$ int $I$ ). Let $J$ be an open interval that satisfies $\bar{J} \subset$ int $I$ for (i) and $\bar{J} \subset$ int $R\left(w^{0}, x_{0}\right)$ for (ii). Then there exists $\varepsilon^{\prime}>0$ such that

$$
R\left(w^{\varepsilon}, x\right) \subset \text { int } I
$$

is valid for all $0<\varepsilon<\varepsilon^{\prime}$ and all $x \in J$ that satisfies $\left(w^{\varepsilon}\right)^{\prime}(x) \in P_{\varepsilon}$ with $\boldsymbol{x} \notin \Sigma\left(w^{\varepsilon}\right)$.

Proof. We first observe that

$$
\left[z_{1}, z_{2}\right]:=\bar{I} \subset\left(a_{-}\left(w^{\epsilon}\right), a_{+}\left(w^{\varepsilon}\right)\right)
$$

for sufficiently small $\varepsilon$, since $\partial w^{e} \rightarrow \partial w^{0}$ in the sense of uppersemiconvergence of (maximal monotone) graphs by Lemma 5.1(ii).

Suppose that the conclusion were false. Then there would exist sequences $\varepsilon_{j} \rightarrow 0$ and $x_{j} \in J \backslash \Sigma\left(w_{j}\right)$ with $\left(w_{j}\right)^{\prime}\left(x_{j}\right) \in P_{\varepsilon_{j}}$ such that $R\left(w_{j}, x_{j}\right)$ contains 
either $\left[\boldsymbol{x}_{j}, z_{2}\right]$ or $\left[z_{1}, \boldsymbol{x}_{j}\right]$ for $j=1,2, \cdots$, where we have set $w_{j}=w^{\varepsilon_{j}}$. We may assume

$$
R\left(w_{j}, x_{j}\right) \supset\left[z_{1}, x_{j}\right]
$$

since the analysis for the other case is symmetric. This inclusion would imply that $w_{j}^{\prime}$ is constant on $\left[z_{1}, x_{j}\right]$. Since $\partial w_{j} \rightarrow \partial w^{0}$ by Lemma 5.1(ii), $\partial w^{0}$ would be constant on $\left[z_{1}, y_{1}\right]$ with $J=\left(y_{1}, y_{2}\right)$.

Thus in the case (ii), $\left(w^{0}\right)^{\prime}$ would be constant on $\left[z_{1}, y_{2}\right]$ since $\operatorname{int} R\left(w^{0}, x_{0}\right)$ $\supset \bar{J}$ implies that $\left(w^{0}\right)^{\prime}$ is constant in a neighbourhood of $\left[y_{1}, y_{2}\right]$. This would contradict the assumption $R\left(w^{0}, x_{0}\right) \subset I$.

In the case (i) $\left(w^{0}\right)^{\prime \prime}$ does not vanish on $I$ since otherwise the weighted curvature of $w^{0}$ would vanish somewhere on $I$, which contradicts a fundamental property of Wulff functions. Since we observed that $\left(w^{0}\right)^{\prime}$ would be constant on $\left[z_{1}, y_{1}\right]$, this now yields a contradiction.

We next extend this property for cutoff Wulff functions so that we handle the piecewise linear region of $w^{0}$ touching the boundary of the domain of definition of $w^{0}$. The next lemma is important to apply Theorem 4.14 in the proof of General stability theorem 2.9.

Lemma 5.3. Let $I$ be a bounded interval with

$$
\text { int } \operatorname{I\cap }\left(\gamma_{-}\left(\tilde{w}^{0}\right), \gamma_{+}\left(\tilde{w}^{0}\right)\right) \neq \emptyset
$$

and let $J$ be an open interval with

$$
\bar{J} \subset\left(\gamma_{-}\left(\tilde{w}^{0}\right), \gamma_{+}\left(\tilde{w}^{0}\right)\right)
$$

Assume the same hypotheses of Lemma 5.2 concerning on $I$ and $J$ by replacing $w^{0}$ by $\tilde{w}^{0}$ (except relation to $a_{ \pm}\left(w^{0}\right)$ ). Then there exists $\varepsilon^{\prime \prime}$ such that

$$
R\left(\tilde{w}^{\varepsilon}, \boldsymbol{x}\right) \subset \text { int } I
$$

is valid for all $0<\varepsilon<\varepsilon^{\prime \prime}$ and all $x \in J \backslash \Sigma\left(\tilde{w}^{\varepsilon}\right)$ that satisfies $\left(\tilde{w}^{\varepsilon}\right)^{\prime}(x) \in P_{\varepsilon}$.

This lemma can be proved along the same line of the proof of Lemma 5.2.

\section{§6. Proof of General stability theorem.}


In this section we shall give the proof of General stability theorem 2.9. Let $P_{\varepsilon}$ be the singularity of $W_{\varepsilon}$ for $\varepsilon \geq 0$. For any $(\varphi,(\hat{t}, \hat{x})) \in A_{P_{0}}(Q) \times Q$ satisfying

$$
\max _{Q}(\bar{u}-\varphi)=(\bar{u}-\varphi)(\hat{t}, \hat{x})
$$

we shall show

$$
g^{\prime}(\hat{t})+F_{0}\left(\hat{t}, f^{\prime}(\hat{z}), \Lambda_{W_{0}}(f, \hat{x})\right) \leq 0
$$

where

$$
\varphi(t, x)=f(x)+g(t), \quad f \in C_{P_{0}}^{2}(\Omega), \quad g \in C^{1}(0, T)
$$

note that $\bar{u}$ is uppersemicontinuous by definition. Without loss of generality we may assume that

$$
(\bar{u}-\varphi)(\hat{t}, \hat{x})=0 \quad \text { and } \quad f^{\prime}(\hat{x})=f(\hat{x})=0
$$

(cf. Proposition 2.7 of [GG3]). Our goal is to prove

$$
g^{\prime}(\hat{t})+F_{0}(\hat{t}, 0, \mu) \leq 0
$$

with $\mu:=\Lambda_{W_{0}}(f, \hat{x})$. As usual we may replace $g(t)$ by $g(t)+(t-\hat{t})^{2}$ still denoted $g(t)$. We may also assume that $u^{\varepsilon}$ for $\varepsilon>0$ is uppersemicontinuous.

Step 1 (the case $\mu \geq 0$ and $f^{\prime \prime}(\hat{x}) \geq 0$ ).

If $0 \in P_{0}$ we may assume that $\chi(f, \hat{x})=1$ and

$$
\bar{u}(\hat{t}, \boldsymbol{x})=f(\hat{x})+g(\hat{t}) \quad \text { for all } \quad x \in R(f, \hat{x})
$$

by Lemma 3.1 .

We may also assume that $\alpha_{\varepsilon} \geq \lambda$ in $\mathbf{R} \backslash P_{\varepsilon}$ for all $\varepsilon \geq 0$ with some $\lambda>0$ independent of $\varepsilon$ by Lemma 3.2, since $\mu \geq 0$.

We shall construct Wulff functions of $\alpha_{\varepsilon}$ with weighted curvature independent of $\varepsilon$ and close to $\mu$, which approximate $f$ near $\hat{\boldsymbol{x}}$. For $\varepsilon \geq 0$ and $\delta>0$, let $w^{\varepsilon, \delta}=w^{\varepsilon, \delta}(x ; \mu+\delta, \tilde{x}, 0)$ be Wulff function of $\alpha_{e}$ with weighted curvature $\mu+\delta$ and top $(\tilde{x}, 0)$ independent of $\varepsilon$, where

$$
\tilde{x}= \begin{cases}\hat{x} & \text { if } \quad 0 \notin P_{0} \\ \{\min R(f, \hat{x})+\max R(f, \hat{x})\} / 2 & \text { if } \quad 0 \in P_{0}\end{cases}
$$


We note that $w^{\varepsilon, \delta} \in C^{1}(\mathbf{R})$ since $\alpha_{\varepsilon}$ is coercive and positive.

For sufficiently small $\delta>0$, say $\delta<\delta_{0}, \bar{u}-\psi^{0, \delta}$ attains its local maximum over $Q$ at $(\hat{t}, x)$ for all $x$ in

$$
S^{\delta}:=\left\{\begin{array}{lll}
\{\tilde{x}\} & \text { if } & 0 \notin P_{0} \\
R\left(w^{0, \delta}, \tilde{x}\right) & \text { if } & 0 \in P_{0}
\end{array}\right.
$$

where we set

$$
\psi^{\varepsilon, \delta}(t, x)=w^{\varepsilon, \delta}(x)+g(t), \quad(t, x) \in Q, \varepsilon \geq 0, \delta>0
$$

Let $I^{\delta}$ be int $R(f, \tilde{x})$ if $0 \in P_{0}$ and be a small open interval in $\Omega$ such that

$$
\begin{aligned}
& \tilde{x} \in I^{\delta}, I^{\delta} \cap \Sigma\left(w^{0, \delta}\right)=\emptyset,\left(w^{0, \delta}\right)^{\prime} \notin P_{0} \text { in } I^{\delta} \\
& f<w^{0, \delta} \text { in } \overline{I^{\delta} \backslash\{\tilde{x}\}}
\end{aligned}
$$

if $0 \notin P_{0}$; we take such an $I^{\delta}$ so that we later apply Lemma 5.2. Since

$$
\begin{cases}f=w^{0, \delta} & \text { in } S^{\delta} \\ f<w^{0, \delta} & \text { in } \overline{I^{\delta} \backslash S^{\delta}},\end{cases}
$$

for each $x \in S^{\delta},(\hat{t}, x)$ is a maximum of $\bar{u}-\psi^{0, \delta}$ over $Q^{\delta}:=(0, T) \times I^{\delta}$.

We fix $\delta, 0<\delta<\delta_{0}$. Lemma 2.16 on convergence of maximum points yields that there exists a sequence $\varepsilon_{j} \downarrow 0$ as $j \rightarrow \infty$ satisfying

$$
\left(t_{j}, x_{j}\right) \rightarrow(\hat{t}, \stackrel{\circ}{x}) \text { as } j \rightarrow \infty \quad \text { with } \quad \stackrel{\circ}{x} \in S^{\delta}
$$

where $\left(t_{j}, x_{j}\right)$ is a maximizer of $u^{\varepsilon_{j}}-\psi^{\varepsilon_{j}, \delta}$ over $Q^{\delta}$, since $w^{\varepsilon, \delta}$ converges to $w^{0, \delta}$ locally uniformly in $\mathbf{R}$ as $\varepsilon \rightarrow 0$ by Lemma 5.1 (i). We denote $w^{\varepsilon_{j}, \delta}$ simply by $w_{j, \delta}$. By the choice of $I^{\delta}$ Lemma 5.2 implies that there exists $j_{0}>0$ such that

$$
R\left(w_{j, \delta}, x\right) \subset I^{\delta}
$$

for all $j>j_{0}$ and all $x \in J^{\delta} \backslash \Sigma\left(w_{j, \delta}\right)$ with $w_{j, \delta}^{\prime} \in P_{\varepsilon_{j}}$ provided that $J^{\delta}$ is an open interval with $\overline{J^{\delta}} \subset I^{\delta}$ containing $S^{\delta}$ if $0 \notin P_{0}$; with $\overline{J^{\delta}} \subset$ int $S^{\delta}$ if $0 \in P_{0}$. We thus obtain

$$
g^{\prime}\left(t_{j}\right)+F_{e_{j}}\left(t_{j}, w_{j, \delta}^{\prime}\left(x_{j}\right), \mu+\delta\right) \leq 0
$$


for $j>j_{0}$ by Theorem 4.5, since $\mu+\delta>0$.

Since the convergence $\left(w^{\varepsilon, \delta}\right)^{\prime} \rightarrow\left(w^{0, \delta}\right)^{\prime}$ is locally uniform in $\mathbf{R}$ by Lemma $5.1($ iii), we have

$$
w_{j, \delta}^{\prime}\left(x_{j}\right) \rightarrow\left(w^{0, \delta}\right)^{\prime}(\hat{x}) \quad \text { as } \quad j \rightarrow \infty
$$

Since $F_{e} \rightarrow F_{0}$ locally uniformly, sending $j$ to infinity in the predeeding inequality yields

$$
g(\hat{t})+F_{0}(\hat{t}, 0, \mu+\delta) \leq 0 .
$$

Since $0<\delta<\delta_{0}$ is arbitrary, we now obtain (6.1).

Step 2 (the case $\mu<0$ ). In this case $\alpha_{0} \neq \equiv 0$ and we may assume that $\alpha_{\varepsilon} \not \equiv 0$ for sufficiently small $\varepsilon>0$. If $0 \in P_{0}$ we may assume that $\hat{x} \in \operatorname{int} R(f, \hat{x})$ and

$$
(\bar{u}-\varphi)(\hat{t}, y)<(\bar{u}-\varphi)(\hat{t}, \hat{x}) \quad \text { for } \quad y \in \partial R(f, \hat{x})
$$

by Lemma 3.1 .

We shall construct (cutoff) Wulff functions of $\alpha_{\varepsilon}$ similarly to Step 1. Since Lemma 3.2 is not applicable for concave functions, we are not allowed to assume coerciveness of $\alpha_{\varepsilon}$ like Step 1, so we should be careful for the domains of definition of Wulff functions.

(a) First we exclude two extreme cases:

$$
\alpha_{0}(p)=0 \text { for all } p<0 \text { and } 0 \in P_{0}
$$

and

$$
\alpha_{0}(p)=0 \text { for all } p>0 \text { and } 0 \in P_{0}
$$

Let $\tilde{z}$ and Wulff function $w^{e, \delta}$ be the same as in Step 1 for $\varepsilon \geq 0$ and $\delta \in(0,-\mu)$. Let $\left(a_{-}\left(w^{\varepsilon, \delta}\right), a_{+}\left(w^{\varepsilon, \delta}\right)\right)$ denote the domain of definition of $w^{\varepsilon, \delta}$ as in Definition 4.3. We observe that $R(f, \tilde{x}) \subset \operatorname{int} R\left(w^{0, \delta}, \tilde{x}\right)$ if $0 \in P_{0}$.

For sufficiently small $\delta>0$, say $\delta<\delta_{0}$ there exists an open interval $I^{\delta}$ containing

$$
\tilde{S}=\left\{\begin{array}{lll}
\{\tilde{x}\} & \text { if } & 0 \notin P_{0} \\
R(f, \hat{x}) & \text { if } & 0 \in P_{0}
\end{array}\right.
$$


such that $\overline{I^{\delta}} \subset\left(a_{-}\left(w^{0, \delta}\right), a_{+}\left(w^{0, \delta}\right)\right) \cap \Omega$ and

$$
\begin{cases}f=w^{0, \delta} & \text { in } \quad \tilde{S} \\ f<w^{0, \delta} & \text { in } \overline{I^{\delta}} \backslash \tilde{S}\end{cases}
$$

and

$$
\begin{cases}\tilde{\boldsymbol{x}} \in I^{\delta}, I^{\delta} \cap \Sigma\left(w^{0, \delta}\right)=\emptyset,\left(w^{0, \delta}\right)^{\prime} \notin P_{0} \text { in } I^{\delta} & \text { if } 0 \notin P_{0} \\ R\left(w^{0, \delta}, \tilde{x}\right) \subset I^{\delta} & \text { if } 0 \in P_{0}\end{cases}
$$

(to apply Lemma 5.2 later). It is possible to find $I^{\delta}$ satisfying (6.5) since we exclude (6.2) and (6.3). (Otherwise one cannot choose such $I^{\delta}$ since boundary of $R\left(w^{0, \delta}, \tilde{x}\right)$ contains $a_{-}\left(w^{0, \delta}\right)$ and/or $a_{+}\left(w^{0, \delta}\right)$ when $0 \notin P_{0}$.) Then $(\hat{t}, x)$ for each $x$ in

$$
S:= \begin{cases}\{\hat{\boldsymbol{x}}\} & \text { if } \quad 0 \notin P_{0}, \\ \{\boldsymbol{x} \in R(f, \hat{\boldsymbol{x}}) ;(\bar{u}-\varphi)(\hat{t}, \boldsymbol{x})=(\bar{u}-\varphi)(\hat{t}, \hat{\boldsymbol{x}})\} & \text { if } \quad 0 \in P_{0}\end{cases}
$$

is a maximizer of $\bar{u}-\psi^{0, \delta}$ in $Q^{\delta}:=(0, T) \times I^{\delta}$, where we set

$$
\psi^{e, \delta}(t, x)=w^{e, \delta}(x)+g(t) \quad(t, x) \in Q^{\delta}, \varepsilon \geq 0, \delta>0
$$

We fix $\delta, 0<\delta<\delta_{0}$. Since $\overline{I^{\delta}} \subset\left(a_{-}\left(w^{\varepsilon, \delta}\right), a_{+}\left(w^{\varepsilon, \delta}\right)\right) \cap \Omega$ for sufficiently small $\varepsilon>0$ by Lemma 5.1 (ii), Lemma 2.16 of convergence of maximum points yields that there exists a sequence $\varepsilon_{j} \downarrow 0$ as $j \rightarrow \infty$ satisfying

$$
\left(t_{j}, x_{j}\right) \rightarrow(\hat{t}, \stackrel{\circ}{x}) \quad \text { as } \quad j \rightarrow \infty \text { with } \quad \stackrel{\circ}{x} \in S
$$

where $\left(t_{j}, x_{j}\right)$ is a maximizer of $u^{\varepsilon_{j}}-\psi^{\varepsilon_{j}, \delta}$ over $Q^{\delta}$, since $w^{\varepsilon, \delta}$ converges to $w^{0, \epsilon}$ locally uniformly in $I^{\delta}$ as $\varepsilon \rightarrow 0$ by Lemma 5.1 (i). We denote $w^{\varepsilon_{j, \delta}}$ simply by $w_{j, \delta}$. By Lemma 5.2 there exists $j_{0}>0$ such that

$$
R\left(w_{j, \delta}, x\right) \subset I^{\delta}
$$

for all $j>j_{0}$ and all $x \in J^{\delta} \backslash \Sigma\left(w_{j, \delta}\right)$ with $w_{j, \delta}^{\prime}(x) \in P_{e_{j}}$, where $J^{\delta}$ is an open interval with $\overline{J^{\delta}} \subset I^{\delta}$ containing $\tilde{\boldsymbol{x}}$ if $0 \notin P_{0}$; with $\overline{J^{\delta}} \subset$ int $R\left(w^{0, \delta}, \tilde{x}\right)$ if $0 \in P_{0}$. Thus Theorem 4.6 yields that

$$
g^{\prime}\left(t_{j}\right)+\max \left\{F_{e_{j}}\left(t_{j}, w_{j, \delta}^{\prime}\left(x_{j}-0\right), \mu+\delta\right), F_{\varepsilon_{j}}\left(t_{j}, w_{j, \delta}^{\prime}\left(x_{j}+0\right), \mu+\delta\right)\right\} \leq 0
$$


for $j>j_{0}$.

We observe that when $0 \notin P_{0}$ the unit Wulff function of $W_{0}^{\prime \prime}$ is $C^{2}$ at 0 , since $\mu<0$ implies $W_{0}^{\prime \prime}(0)=W_{0}^{\prime \prime}\left(f^{\prime}(\hat{x})\right)>0$; when $0 \in P_{0}$ we have $S \subset$ int $R(f, \hat{x})$. Thus we see $S \cap \Sigma\left(w^{0, \delta}\right)=\emptyset$, which yields that

$$
w_{j, \delta}^{\prime}\left(\boldsymbol{x}_{j} \pm 0\right) \rightarrow\left(w^{0, \delta}\right)^{\prime}(\stackrel{\circ}{x})=0 \quad \text { as } \quad j \rightarrow \infty
$$

by Lemma 5.1. Sending $j$ to infinity in the last inequality yields that

$$
g^{\prime}(\hat{t})+F_{0}(\hat{t}, 0, \mu+\delta) \leq 0
$$

since $F_{\varepsilon} \rightarrow F_{0}$ is locally uniform. Since $\delta>0$ is arbitrary small, we get (6.1).

(b) Using cutoff Wulff functions instead of Wulff functions, we shall show (6.1) for general cases including (6.2) and (6.3). Although the proof includes case (a), we have presented the proof of case (a) separately to show our main idea avoiding technicalities.

Let $q_{-}$and $q_{+}$be a negative and positive number, respectively. For $\varepsilon \geq 0$ and $\delta \in(0,-\mu)$, let $\tilde{w}^{e, \delta}=\tilde{w}^{e, \delta}\left(x ; \mu+\delta, \tilde{x}, 0, q_{-}, q_{+}\right)$be a cutoff Wulff function of $\alpha_{\varepsilon}$ with weighted curvature $\mu+\delta$, top $(\tilde{x}, 0)$ and with slopes $\left(q_{-}, q_{+}\right)$, where $\tilde{\boldsymbol{x}}$ is as in (a). By definition $\tilde{w}^{\varepsilon, \delta} \in C(\mathbf{R})$.

Let $\tilde{S}$ be as in (a). We see that $\tilde{S} \subset\left(\gamma_{-}\left(\tilde{w}^{0, \delta}\right), \gamma_{+}\left(\tilde{w}^{0, \delta}\right)\right)$, where $\gamma_{ \pm}$is as in Definition 4.12. For sufficiently small $\delta>0$, say $\delta<\delta_{0}$, there is an open interval $I^{\delta}$ with $\overline{I^{\delta}} \subset \Omega$ containing $\tilde{S}$ satisfying (6.4) and (6.5) replacing $w^{0, \delta}$ by $\tilde{\boldsymbol{w}}^{0, \delta}$. Since we take a cutoff Wulff function instead of a Wulff function, we can choose such $I^{\delta}$ even for (6.2) and/or (6.3). Then $\bar{u}-\psi^{0, \delta}$ attains its maximum over $Q^{\delta}:=(0, T) \times I^{\delta}$ at $(\hat{t}, x)$ for all $x$ in $S$, where $S$ is as in (a) and we set

$$
\psi^{\varepsilon, \delta}(t, x)=\tilde{w}^{\varepsilon, \delta}(x)+g(t), \quad(t, x) \in Q^{\delta}, \varepsilon \geq 0, \delta>0
$$

We fix $\delta, 0<\delta<\delta_{0}$. There exists a sequence $\left\{\varepsilon_{j}\right\}$ as in (a) by Lemma 2.6, since $\tilde{w}^{e, \delta}$ converges to $\tilde{w}^{0, \delta}$ uniformly in $I^{\delta}$ as $\varepsilon \rightarrow 0$ by Lemma 5.1 (iv). Lemma 5.3 yields that there exists $j_{0}>0$ such that

$$
R\left(\tilde{w}_{j, \delta}, \boldsymbol{x}\right) \subset I^{\delta}
$$


for all $j>j_{0}$ and all $x \in J^{\delta} \backslash \Sigma\left(\tilde{w}_{j, \delta}\right)$ with $\tilde{w}_{j, \delta}^{\prime}(x) \in P_{\varepsilon_{j}}$, where we denote $\tilde{w}^{\varepsilon_{j}, \delta}$ by $\tilde{w}_{j, \delta}$ and where open interval $J^{\delta}$ is as in (a) replacing $w_{j, \delta}$ by $\tilde{w}_{j, \delta}$, with extra assumption $\overline{J^{\delta}} \subset\left(\gamma_{-}\left(\tilde{w}^{0, \delta}\right), \gamma_{+}\left(\tilde{w}^{0, \delta}\right)\right)$ when $0 \notin P_{0}$. By the convergence of $\tilde{w}_{j, \delta}$ to $\tilde{w}^{0, \delta}$ in Lemma 5.1 (iv), $S \subset\left(\gamma_{-}\left(\tilde{w}^{0, \delta}\right), \gamma_{+}\left(\tilde{w}^{0, \delta}\right)\right)$ implies that $x_{j} \in\left(\gamma_{-}\left(\tilde{w}_{j, \delta}\right), \gamma_{+}\left(\tilde{w}_{j, \delta}\right)\right)$ and that $R\left(\tilde{w}_{j, \delta}, x_{j} \pm 0\right) \subset I$ for $j>j_{1}$ with sufficiently large $j_{1} \geq j_{0}$. We now apply Theorem 4.14 to obtain

$$
g^{\prime}\left(t_{j}\right)+\max \left\{F_{e_{j}}\left(t_{j}, \tilde{w}_{j, \delta}^{\prime}\left(\boldsymbol{x}_{j}-0\right), \mu+\delta\right), F_{e_{j}}\left(t_{j}, \tilde{w}_{j, \delta}^{\prime}\left(\boldsymbol{x}_{j}+0\right), \mu+\delta\right)\right\} \leq 0
$$

for $j>j_{1}$.

Since Lemma 5.1 (iv) and $S \cap \Sigma\left(\tilde{w}^{0, \delta}\right)=\emptyset$ implies that

$$
\tilde{w}_{j, \delta}^{\prime}\left(x_{j} \pm 0\right) \rightarrow\left(\tilde{w}^{0, \delta}\right)^{\prime}(\stackrel{\circ}{x})=0 \quad \text { as } \quad j \rightarrow \infty \quad \text { with } \quad \stackrel{\circ}{x} \in S,
$$

sending $j$ to infinity yields

$$
g^{\prime}(\hat{t})+F_{0}(\hat{t}, 0, \mu+\delta) \leq 0
$$

which as in (a) yields $(6.1)$ by sending $\delta \rightarrow 0$.

Step 3 (the case $\mu=0$ and $f^{\prime \prime}(\hat{\boldsymbol{x}})<0$ ).

By Lemma 3.1 we may assume $0 \notin P_{0}$. There is function $h \in C^{2}(\Omega)$ with an open neighborhood $I \subset \Omega$ of $\hat{x}$ such that

$$
\left\{\begin{array}{lll}
h=0 & \text { in } & \bar{J} \\
h^{\prime}>0 & \text { in } & I \backslash \bar{J}, \\
h>f & \text { in } & \bar{I} \backslash\{\hat{\boldsymbol{x}}\}
\end{array}\right.
$$

where $J$ is an open neighborhood of $\hat{\boldsymbol{z}}$ with $\bar{J} \subset I$. Thus we see that $\bar{u}-\psi$ attains its strict maximum over $(0, T) \times I$ at $(\hat{t}, \hat{x})$ where

$$
\psi(t, x)=h(x)+g(t), \quad(t, x) \in(0, T) \times I .
$$

Lemma 2.16 yields that there exists a sequence $\varepsilon_{j} \downarrow 0$ as $j \rightarrow \infty$ such that

$$
\left(t_{j}, x_{j}\right) \rightarrow(\hat{t}, \hat{x}) \quad \text { as } \quad j \rightarrow \infty
$$

where $\left(t_{j}, x_{j}\right)$ is a maximizer of $u^{\varepsilon_{j}}-\psi$ over $(0, T) \times I$. We obtain

$$
g^{\prime}\left(t_{j}\right)+F_{e_{j}}\left(t_{j}, h^{\prime}\left(x_{j}\right), \Lambda_{W_{\bullet_{j}}}\left(h, x_{j}\right)\right) \leq 0
$$


by Lemma 2.18. We observe that $\Lambda_{W_{s_{j}}}\left(h, x_{j}\right) \rightarrow 0$ as $j \rightarrow \infty$ since $h^{\prime}\left(x_{j}\right)$ and $h^{\prime \prime}\left(x_{j}\right) \rightarrow 0$ as $j \rightarrow \infty$ and if $0 \in P_{e_{j}}$ we have $\chi\left(h, x_{j}\right)=0$. Sending $j$ to infinity, we obtain (6.1).

Remark. If solution $\boldsymbol{u}^{\varepsilon}$ is uniformly Lipschitz in $\boldsymbol{x} \in \Omega$ for $0<\varepsilon<1,0<$ $t<T$, so that

$$
\sup _{0<\varepsilon<1} \operatorname{ess.sup}\left|u_{x}^{e}\right|=M<\infty, \quad Q=(0, T) \times \Omega
$$

then the proof of Step 2 is simplified. Since we may modify the value of $\alpha_{\varepsilon}(\varepsilon \geq 0)$ for $p,|p| \geq M+1$ so that new $\alpha_{\varepsilon}$ is coersive, case (b) of Step 2 is unnecessary and case (a) is simplified since $w^{\varepsilon, \delta}$ is defined in $\mathbf{R}$.

\section{$\S 7$. Construction of barriers.}

We shall prove Theorem 2.11 on convergence of solutions. The main task in this section is to prove $\bar{u} \leq \underline{u}$ at $t=0$ by constructing suitable barriers with use of cutoff Wulff functions, where $\bar{u}=\limsup _{\varepsilon \rightarrow 0}^{*} u^{\varepsilon}$ and $\underline{u}=\liminf _{\varepsilon \rightarrow 0} u^{\varepsilon}$. Such barriers are useful to construct solutions by Perron's method as in [GG4].

Lemma 7.1 (Curvature equation and cutoff Wulff functions). For $\mu>0, \tilde{x}, \tilde{y} \in \mathbf{R}, q_{-}<0<q_{+}$let

$$
\tilde{w}(x)=\tilde{w}\left(x ; \mu, \tilde{x}, \tilde{y}, q_{-}, q_{+}\right)
$$

be a cutoff $W u l f f$ function of $\alpha \in \mathcal{A}$ with weighted curvature $\mu$, slopes $\left(q_{-}, q_{+}\right)$ and top $(\tilde{x}, \tilde{y})$. If $(f, \hat{x}) \in C_{P}^{2}(\mathbf{R}) \times \mathbf{R}$ satisfies

$$
\min _{\mathbf{R}}(\tilde{w}-f)=(\tilde{w}-f)(\hat{x})
$$

then it holds

$$
-\Lambda_{W}(f, \hat{x})+\mu \geq 0
$$

Here $P$ denotes the singularity of $W$.

Proof. We may assume that $\alpha \not \equiv 0$ since otherwise $\Lambda_{W} \equiv 0$ so (7.1) is trivial. Let $\hat{p}$ denote $f^{\prime}(\hat{\boldsymbol{x}})$. 
Case $1\left(\hat{p} \in P\right.$ and $\left.\hat{x} \in\left(\gamma_{-}(\tilde{w}), \gamma_{+}(\tilde{w})\right)\right)$.

(A) If $\hat{x} \notin \Sigma(\tilde{w})$ then we see that

$$
\Lambda_{W}(f, \hat{x}) \leq \Lambda_{W}(\tilde{w}, \hat{x})
$$

since $\tilde{w}$ is convex and $R(f, \hat{x}) \supset R(\tilde{w}, \hat{x})$ when $\chi(f, \hat{x})=1$. By Proposition 4.13 we have $\Lambda_{W}(\tilde{w}, \hat{x})=\mu$. This together with (7.2) yields (7.1).

(B) If $\hat{x} \in \Sigma(\tilde{w})$ then $\tilde{w}^{\prime}(\hat{x}-0)=\hat{p}$ or $\tilde{w}^{\prime}(\hat{x}+0)=\hat{p}$ by the definition of a cutoff Wulff function. If there exists $\hat{y} \in$ int $R(f, \hat{x})$ such that $(\tilde{w}-f)(\hat{x})=$ $(\tilde{w}-f)(\hat{y})$, we apply $(\mathbf{A})$ to $\hat{y}$ instead of $\hat{x}$ to get

$$
\Lambda_{W}(f, \hat{x})=\Lambda_{W}(f, \hat{y}) \leq \Lambda_{W}(\tilde{w}, \hat{y})=\mu
$$

Otherwise, $\Lambda_{W}(f, \hat{x}) \leq 0$ since $\chi(f, \hat{x}) \leq 0$. In any case $(7.1)$ follows.

Case $2 \quad\left(\hat{p} \notin P\right.$ and $\left.\hat{x} \in\left(\gamma_{-}(\tilde{w}), \gamma_{+}(\tilde{w})\right)\right)$.

(A) If $\hat{x} \notin \Sigma(\tilde{w})$ then we see that inequality (7.2) holds since $\hat{p}=\tilde{w}^{\prime}(\hat{x})$ and $f^{\prime \prime}(\hat{\boldsymbol{x}}) \leq \tilde{w}^{\prime \prime}(\hat{\boldsymbol{x}})$. Proposition 4.13 yields that $\Lambda_{W}(\tilde{w}, \hat{\boldsymbol{x}})=\mu$. Thus (7.1) follows.

(B) If $\hat{\boldsymbol{x}} \in \Sigma(\tilde{w})$ then $W^{\prime \prime}(\hat{p})=0$ by Proposition 4.4 (ii) since $\hat{p} \in\left[\tilde{w}^{\prime}(\hat{x}-\right.$ $\left.0), \tilde{w}^{\prime}(\hat{x}+0)\right]$. Thus we obtain $\Lambda_{W}(f, \hat{x})=0$ which yields $(7.1)$.

Case $3 \quad\left(\hat{x} \notin\left[\gamma_{-}(\tilde{w}), \gamma_{+}(\tilde{w})\right]\right)$. Since $\tilde{w}$ is affine outside $\left[\gamma_{-}(\tilde{w}), \gamma_{+}(\tilde{w})\right]$, if $\hat{p} \in P$ then $\chi(f, \hat{x}) \leq 0$ to get $\Lambda_{W}(f, \hat{x}) \leq 0$; if $\hat{p} \notin P$ then $\Lambda_{W}(f, \hat{x})=0$ since $f^{\prime \prime}(\hat{x})=0$. Evidently, (7.1) follows.

Case $4\left(\hat{\boldsymbol{x}}=\gamma_{-}(\tilde{\boldsymbol{w}})\right.$ or $\left.\gamma_{+}(\tilde{w})\right)$. We prove only when $\hat{\boldsymbol{x}}=\gamma_{-}(\tilde{w})$ since the argument of the case $\hat{x}=\gamma_{+}(\tilde{w})$ is symmetric.

If $q_{-}=\hat{p} \in P$ then as in Case $3 \chi(f, \hat{x}) \leq 0$, so that $\Lambda_{W}(f, \hat{x}) \leq 0$.

If $\tilde{w}(\hat{z}+0)=\hat{p} \in P$ then similar argument as in Case 1 (B) yields (7.2).

If $\hat{p} \notin P$ and $W^{\prime \prime}(\hat{p})=0$, then $\Lambda_{W}(f, \hat{x})=0$.

If $\hat{p} \notin P$ and $W^{\prime \prime}(\hat{p})>0$, then $\tilde{w}$ is $C^{1}$ at $\hat{x}=\gamma_{-}(\tilde{w})$. Since $f^{\prime \prime}(\hat{x}) \leq$ $\min \left\{\tilde{\boldsymbol{w}}^{\prime \prime}(\hat{\boldsymbol{x}}-0), \tilde{\boldsymbol{w}}^{\prime \prime}(\hat{\boldsymbol{x}}+0)\right\}=0, \Lambda_{W}(f, \hat{\boldsymbol{x}}) \leq 0$ holds. In any cases $(7.1)$ follows. 
Lemma 7.2 (Fundamental supersolutions). Assume that $F \in \mathcal{F}$. For $W \in \mathcal{E}$, let

$$
G(x):=\tilde{w}\left(x ; \mu, 0,0, q_{-}, q_{+}\right), \quad x \in \mathbf{R}
$$

be a cutoff Wulff function of $\alpha=W^{\prime \prime}(\not \equiv 0) \in \mathcal{A}$ with parameters $\mu>0, q_{-}<$ 0 and $q_{+}>0$. For $S$ in $(0, T)$ there exists (large) $\bar{B} \geq 0$ depending only on $F, \mu, q_{-}$and $q_{+}$such that

$$
G^{+}(t, x):=B t+G(x) \text { for }(t, x) \in \bar{Q}_{S} \text { and } B \geq \bar{B}
$$

is a supersolution of $(E)$ in $Q_{S}:=(0, S) \times \mathbf{R}$,

Proof. Suppose that $(\varphi,(\hat{t}, \hat{x})) \in A_{P}\left(Q_{S}\right) \times Q_{S}$ satisfies

$$
\min _{Q}\left(G^{+}-\varphi\right)=\left(G^{+}-\varphi\right)(\hat{t}, \hat{x})
$$

and that $\varphi$ is of the form

$$
\varphi(t, x)=f(x)+g(t), \quad(t, x) \in Q_{S},
$$

with $f \in C_{P}^{2}(\mathbf{R})$ and $g \in C^{1}(0, T)$, where $P$ denotes the singularity of $W$. We observe that $\Lambda_{W}(f, \hat{x}) \leq \mu$ by Lemma 7.1. By (F1)

$$
c:=\min \left\{F(t, p, \mu) ; t \in[0, S], p \in\left[q_{-}, q_{+}\right]\right\}>-\infty .
$$

Since $F$ is degenerate elliptic by $(\mathrm{F} 2), f^{\prime}(\hat{x}) \in\left[q_{-}, q_{+}\right]$implies

$$
F\left(t, f^{\prime}(\hat{x}), \Lambda_{W}(f, \hat{x})\right) \geq c \text { for } t \in[0, S] .
$$

Choosing $\bar{B} \geq \max \{-c, 0\}$, it holds that

$$
\begin{aligned}
& \varphi_{t}(\hat{t}, \hat{x})+F\left(\hat{t}, \varphi_{x}(\hat{t}, \hat{x}), \Lambda_{W}(\varphi(\hat{t}, \cdot), \hat{x})\right) \\
& \geq B+F\left(\hat{t}, f^{\prime}(\hat{x}), \mu\right) \geq B+c \geq B-\bar{B} \geq 0 \quad \text { for } \quad B \geq \bar{B} .
\end{aligned}
$$

Since $\left(\left.G^{+}\right|_{Q_{S}}\right)_{*} \geq 0>-\infty, G^{+}$is now a supersolution of $(\mathrm{E})$ in $Q_{S}$.

Remark 7.3. Since $G$ is convex and $F$ is degenerate elliptic, $G^{+}$is also a subsolution of

$$
u_{t}+F\left(t, u_{x}, 0\right)=0
$$


Lemma 7.4. Let $M$ and $\delta$ be given positive numbers. Assume that $W \in$ $\mathcal{E}$. Then there exist $\mu>0, q_{-}<0$ and $q_{+}>0$ such that the cutoff Wulff function $\tilde{w}$ of $\alpha$ with weighted curvature $\mu$, slopes $\left(q_{-}, q_{+}\right)$and top $(0,0)$ satisfies

$$
\tilde{w}\left(x ; \mu, 0,0, q_{-}, q_{+}\right) \geq M \quad \text { for } \quad|x| \geq \delta
$$

Proof. We may assume $\alpha \neq 0$ since the case $\alpha \equiv 0$ is easy to handle. We take $q_{-}$and $q_{+}$such that

$$
q_{-} \leq-2 M / \delta \text { for } q_{+} \geq 2 M / \delta \text {. }
$$

Choose $\mu$ as

$$
\mu>\max \left\{-2 x_{1} / \delta, 2 x_{2} / \delta\right\}
$$

with $x_{1}=\max \left\{x ; \inf \partial \stackrel{\circ}{w}(x) \leq q_{-}\right\}, x_{2}=\min \left\{x ; \sup \partial \stackrel{\circ}{w}(x) \geq q_{+}\right\}$, where $\stackrel{\circ}{w}$ denotes the unit Wulff function of $\alpha$. Here as in $\S 5$ with abuse of the notation $\partial \stackrel{\circ}{w}$ denotes the subdifferential of the lower semicontinuous extension of $\stackrel{\circ}{w}$ with values $\infty$ outside $\left(\beta_{-}, \beta_{+}\right)$so that $\partial \stackrel{\circ}{w}$ is a maximal monotone operator (denoted $\beta^{-1}$ in Definition 4.1). Since the range of $\beta^{-1}$ equals $\mathbf{R}$, we have

$$
\lim _{x \downarrow \beta_{-} \beta_{-} \leq z \leq x} \inf _{\dot{w}} \dot{o}(z)=-\infty \text { and } \lim _{\boldsymbol{x} \uparrow \beta_{+}} \sup _{x \leq z \leq \beta_{+}} \partial \stackrel{\circ}{w}(z)=+\infty .
$$

We observe that $-\infty<x_{1} \leq 0$ and $0 \leq x_{2}<\infty$. Let

$$
\tilde{\dot{o}}(\boldsymbol{x}):=\tilde{\mathrm{o}} w\left(\boldsymbol{x} ; q_{-}, q_{+}\right), \quad \boldsymbol{x} \in \mathbf{R}
$$

denote a cutoff unit Wulff function of $\alpha$. Since

$$
\stackrel{\circ}{w}^{\prime}\left(x ; q_{-}, q_{+}\right)= \begin{cases}q_{-}, & x<x_{1} \\ q_{+}, & x>x_{2}\end{cases}
$$

and

$$
\tilde{\boldsymbol{w}}^{\prime}\left(\boldsymbol{x} ; \mu, 0,0, q_{-}, q_{+}\right)=\tilde{\stackrel{o}{ }}^{\prime}\left(\mu x ; q_{-}, q_{+}\right), \boldsymbol{x} \in \mathbf{R}
$$

as observed right after Definition 4.12, we have

$$
\tilde{w}\left(x ; \mu, 0,0, q_{-}, q_{+}\right) \geq \int_{\delta / 2}^{\delta} \stackrel{\tilde{o}^{\prime}}{w}\left(\mu y ; q_{-}, q_{+}\right) d y=q_{+} \cdot \delta / 2 \geq M \quad \text { for } \quad x \geq \delta
$$

and similarly

$$
\tilde{w}\left(x ; \mu, 0,0, q_{-}, q_{+}\right) \geq M \text { for } x \leq-\delta .
$$


Lemma 7.5. Let $M$ and $\delta$ be positive numbers. Assume that $\alpha_{\varepsilon}=$ $W_{\varepsilon} " \in \mathcal{A}$ converges to $\alpha_{0}=W_{0}^{\prime \prime} \in \mathcal{A}$ weakly as measures as $\varepsilon \rightarrow 0$ (with $\left.W_{\varepsilon}, W \in \mathcal{E}\right)$. Then there exist $\bar{\varepsilon}>0, \mu>0, q_{-}<0$ and $q_{+}>0$ independent of $\varepsilon$ such that cutoff Wulff function $\tilde{w}^{\varepsilon}$ of $\alpha_{\varepsilon}$ with weighted curvature $\mu$, slopes $\left(q_{-}, q_{+}\right)$and top $(0,0)$ satisfies

$$
\tilde{w}^{e}\left(x ;, \mu, 0,0, q_{-}, q_{+}\right) \geq M \quad \text { for } \quad|x| \geq \delta \quad \text { and } \varepsilon \in[0, \bar{\varepsilon}]
$$

Proof. We first assume that $\alpha_{\varepsilon} \not \equiv 0$ and $\alpha_{0} \not \equiv 0$. We take $q_{-}$and $q_{+}$ satisfying

$$
q_{-}<-2 M / \delta \text { and } q_{+}>2 M / \delta
$$

Let $\dot{w}^{\varepsilon}$ denote the unit Wulff function of $\alpha_{e}$ for $\varepsilon \geq 0$. We take a bounded rectangular domain $C=\left(c_{1}, c_{2}\right) \times\left(c_{3}, c_{4}\right) \in \mathbf{R}^{2}$ such that

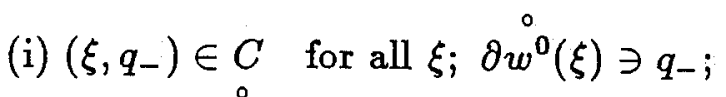

(ii) graph $\partial w^{0} \cap$ ( the boundary of $C$ ) consists of two points, say, $\left(\xi_{1}, \eta_{1}\right)$ and $\left(\xi_{2}, \eta_{2}\right)$ with $\xi_{1} \leq \xi_{2}$ and $\eta_{1} \leq \eta_{2}$.

Here graph $\partial \stackrel{\circ}{w}^{0}$ denotes $\left\{(\xi, q) ; q \in \partial \stackrel{\circ}{w^{0}}(\xi)\right\}$. Such $C$ does exist since $\partial{ }^{\circ}$ is a maximal monotone operator and whose range equals $\mathbf{R}$.

We observe that $c_{1}<0$ since $q_{-}<0$ and $\partial{w^{0}}^{0}(0) \ni 0$. Setting $D_{\nu}=\{(\xi, \eta)$; dist (graph $\left.\left.\partial \tilde{w}^{0},(\xi, \eta)\right)<\nu\right\}$ for $\nu>0$, there exists $\nu_{0}>0$ such that

$$
\xi^{\nu}:=\inf \left\{\xi \in \mathbf{R} ;\left(\xi, q_{-}\right) \in D_{\nu} \cap C\right\}>c_{1} \quad \text { for } \quad \nu \in\left(0, \nu_{0}\right]
$$

Since $\partial w^{\varepsilon}$ converges to $\partial w^{0}$ in the sense of uppersemiconvergence of graphs $\left(\right.$ Limsup $_{\varepsilon \rightarrow 0}\left(\right.$ graph $\left.\partial w^{\varepsilon}\right) \subset$ graph $\left.\partial w^{0}\right)$ by Lemma 5.1 (ii), there exists $\varepsilon_{1}=$ $\varepsilon_{1}\left(\nu_{0}\right)>0$ such that

$$
\text { (graph } \left.\partial \stackrel{0}{w^{\varepsilon}}\right) \cap C \subset D_{\nu_{0}} \cap C \text { for all } \varepsilon \in\left(0, \varepsilon_{1}\right) \text {. }
$$

Thus we see

$$
\max \left\{x ; \inf \partial \dot{w}^{e}(x) \leq q_{-}\right\} \geq \xi^{\nu}>c_{1} \text { for all } \varepsilon \in\left[0, \varepsilon_{1}\right)
$$


since $q_{-} \in\left(c_{3}, c_{4}\right)$. Similarly there exists $\varepsilon_{2}>0$ and $c_{1}^{\prime} \geq 0$ such that

$$
\min \left\{x, \sup \partial \stackrel{\circ}{w}^{\varepsilon}(x) \geq q_{+}\right\}<c_{1}^{\prime} \text { for all } \varepsilon \in\left[0, \varepsilon_{2}\right)
$$

We take $x_{1}, x_{2}$ and $\mu$ independently of $\varepsilon$ that satisfies

$$
x_{1} \leq c_{1} \quad \text { and } \quad x_{2} \geq c_{1}^{\prime}
$$

and $\mu>\max \left\{-2 x_{1} / \delta, 2 x_{2} / \delta\right\}$, and argue as in the proof of Lemma 7.4. By (7.3) and (7.4) we observe that for all $\varepsilon \in\left[0, \min \left\{\varepsilon_{1}, \varepsilon_{2}\right\}\right]$

$$
\tilde{w}^{e}\left(x ; \mu, 0,0, q_{-}, q_{+}\right) \geq M \quad \text { for } \quad|x| \geq \delta
$$

since $c_{1}$ and $c_{1}^{\prime}$ are independent of $\varepsilon$.

Even if $\alpha_{\varepsilon} \equiv 0$ for some $\varepsilon \geq 0$, the proof is still valid by interpreting $\partial \dot{o}^{e}(0)=\mathbf{R}$ and $\partial \dot{\circ}^{e}(x)=\emptyset$ for $x \neq 0, \varepsilon \geq 0$.

We shall construct a "barrier" of the solution of $(\mathrm{E})$ in the following lemma. To do this we prepare some notation. Let $u_{0} \in C(\mathbf{R})$ be periodic with period $\varpi$. Since $u_{0}$ is uniformly continuous, for each $\sigma \in(0,1)$ there exists $\delta=\delta(\sigma) \in(0,1 / 2)$ such that

$$
\left|u_{0}(x)-u_{0}(\xi)\right|<\sigma \quad \text { for } \quad|x-\xi|<\delta .
$$

For $W \in \mathcal{E}$ with $W^{\prime \prime} \not \equiv 0$ we take the cutoff Wulff function of $W^{\prime \prime}$ :

$$
G^{\sigma}(x)=\tilde{w}\left(x ; \mu, 0,0, q-, q_{+}\right) \geq M \quad \text { for }|x| \geq \delta
$$

which is chosen in Lemma 7.4 with $\delta=\delta(\sigma)$ and $M$ chosen so that $M \geq$ $\max u_{0}-\min u_{0}$ independent of $\sigma$. For $F \in \mathcal{F}$ let $G^{+, \sigma}$ be a supersolution of $(\mathrm{E})$ in $Q_{S}:=(0, S) \times \mathbf{R}$ defined in Lemma 7.2 with $G=G^{\sigma}$ with some large $B$.

Lemma 7.6 (Barriers). Assume that $F \in \mathcal{F}_{\#}$ and $W \in \mathcal{E}$ with $W^{\prime \prime} \not \equiv 0$. Assume that $u_{0} \in C(\mathbf{R})$ is periodic with period $\varpi>0$. Then for each $\xi \in \mathbf{R}$ and $\sigma \in(0,1)$,

$$
\tilde{G}^{+, \sigma}(t, x ; \xi):=G^{+, \sigma}(t, x-\xi)+u_{0}(\xi)+\sigma \quad \text { for } \quad(t, x) \in \bar{Q}_{S}
$$


and

$$
\hat{G}^{+, \sigma}(t, x ; \xi):=\inf \left\{\tilde{G}^{+, \sigma}(t, x ; \eta) ; \eta-\xi \in \varpi \mathbf{Z}\right\} \quad \text { for } \quad(t, x) \in \bar{Q}_{S}
$$

are supersolutions of $(E)$ in $Q_{s}:=(0, S) \times \mathbf{R}$ with $S \in(0, T)$. Moreover, the generalized solution $u \in C([0, T) \times \mathbf{R})$ of $(E)$ in $(0, T) \times \mathbf{R}$ with

$$
u(0, x)=u_{0}(x), \quad x \in \mathbf{R}
$$

satisfies

$$
u \leq \hat{G}^{+, \sigma} \quad \text { in } \quad[0, S) \times \mathbf{R} .
$$

Proof. We evidently observe that $\tilde{G}^{+, \sigma}$ and $\hat{G}^{+, \sigma}$ are continuous and bounded. It is clear that $\tilde{G}^{+, \sigma}$ is a supersolution of (E) since $G^{+, \sigma}$ is a supersolution. Lemma 8.1' in [GG4] yields that $\hat{G}^{+, \sigma}$ is also a supersolution of $(\mathrm{E})$.

Since $u_{0}$ is uniformly continuous, by definition of $\delta$ we have

$$
u_{0}(x)<u_{0}(\xi)+\sigma \text { for }|x-\xi|<\delta(\sigma)
$$

The definition of $G^{+, \sigma}$ and the choice of $M$ yield

$$
u_{0}(x) \leq u_{0}(\xi)+M \leq u_{0}(\xi)+G^{+, \sigma}(0, x ; \xi) \text { for }|x-\xi| \geq \delta(\sigma)
$$

for each $\xi \in \mathbf{R}$ and $\sigma \in(0,1)$ we have

$$
u_{0}(x) \leq \tilde{G}^{+, \sigma}(0, x ; \xi) \text { for } \quad x \in \mathbf{R}
$$

so that

$$
u_{0}(x) \leq \hat{G}^{+, \sigma}(0, x ; \xi) \text { for } x \in \mathbf{R} .
$$

Since $\hat{G}^{+, \sigma}$ is periodic in $\approx$ with period $\varpi$ and $F \in \mathcal{F}_{\#}$, Comparison theorem 2.7 yields that for each $\xi \in \mathbf{R}$ and $\sigma \in(0,1)$

$$
u \leq \hat{G}^{+, \sigma} \text { in } Q_{S} .
$$


Lemma 7.7. Assume that same hypotheses as Theorem 2.11. Then it holds that

$$
\begin{aligned}
& \bar{u}(0, x) \leq u_{0}(x) \quad \text { for } \quad x \in \mathbf{R}, \\
& \underline{u}(0, x) \geq u_{0}(x) \quad \text { for } \quad x \in \mathbf{R}
\end{aligned}
$$

where $\bar{u}=\limsup _{\varepsilon \downarrow 0} u^{\varepsilon}$ and $\underline{u}=\liminf _{\varepsilon \downarrow 0} u^{e}$.

Proof. Step 1. We shall construct supersolution $G_{e}^{+, \sigma}$ of $\left(E_{e}\right)$ from cutoff Wulff function $\tilde{w}^{e}$ of $\alpha_{\varepsilon}$.

Since $u_{0}^{\varepsilon}$ converges to $u_{0}$ uniformly as $\varepsilon \rightarrow 0$, there exists modulus $\omega$ of $u_{0}^{\varepsilon}$ independent of $\varepsilon$, i.e., $\left|u_{0}^{\varepsilon}(x)-u_{0}^{\varepsilon}(y)\right|<\omega(|x-y|)$ for all $\varepsilon \in[0,1)$. For convenience we denote $u_{0}$ also by $u_{0}^{0}$. We see that for all $\sigma \in(0,1)$ there exists $\delta=\delta(\sigma)>0$ such that $\omega(\delta)<\sigma$.

By Lemma 7.5 for each $\sigma \in(0,1)$ there exist $\bar{\varepsilon}^{\sigma} \in(0,1), \mu^{\sigma}>0, q_{-}^{\sigma}<0$ and $q_{+}^{\sigma}>0$ such that cutoff Wulff function $\tilde{w}^{\varepsilon}$ of $\alpha_{\varepsilon}$ satisfies

$$
G_{\varepsilon}^{\sigma}(x):=\tilde{w}^{e}\left(x ; \mu^{\sigma}, 0,0, q_{-}^{\sigma}, q_{+}^{\sigma}\right) \geq M \quad \text { for } \quad|x| \geq \delta(\sigma) \text { and } \varepsilon \in\left[0, \bar{\varepsilon}^{\sigma}\right]
$$

with

$$
M \geq \sup \left\{\sup _{\mathbf{R}} u_{0}^{\varepsilon}-\inf _{\mathbf{R}} u_{0}^{\varepsilon} ; \varepsilon \in\left[0, \bar{\varepsilon}^{\sigma}\right]\right\} .
$$

Let $S \in(0, T)$ be fixed. Lemma 7.2 yields that for $\varepsilon \in\left[0, \bar{\varepsilon}^{\sigma}\right]$ there exists $\bar{B}_{\epsilon}^{\sigma} \geq 0$ such that

$$
G_{e}^{+, \sigma}:=B^{\sigma} t+G_{\varepsilon}^{\sigma}(x) \text { for }(t, x) \in \bar{Q}_{S} \text { and } B^{\sigma} \geq \bar{B}_{\varepsilon}^{\sigma}
$$

is a supersolution of $\left(E_{\varepsilon}\right)$ in $Q_{S}$.

Since $\bar{B}^{\sigma}:=\sup \left\{\bar{B}_{\varepsilon}^{\sigma} ; \varepsilon \in\left[0, \bar{\varepsilon}^{\sigma}\right]\right\}$ is finite we choose $B^{\sigma} \geq \bar{B}^{\sigma}$ independent of $\varepsilon$ in the definition of $G_{e}^{+, \sigma}$.

Step 2. We shall construct barriers $\hat{G}_{\varepsilon}^{+, \sigma}$. For each $\sigma \in(0,1), \varepsilon \in\left[0, \bar{\varepsilon}^{\sigma}\right]$ and $\xi \in \mathbf{R}$,

$\hat{G}_{\varepsilon}^{+, \sigma}(t, x ; \xi):=\inf \left\{G_{\varepsilon}^{+, \sigma}(t, x-\eta)+u_{0}^{\varepsilon}(\eta)+\sigma ; \eta-\xi \in \varpi \mathbf{Z}\right\} \quad$ for $\quad(t, x) \in \bar{Q}_{S}$

is a supersolution of $\left(E_{\varepsilon}\right)$ in $Q_{S}$ and

$$
u^{\varepsilon} \leq \hat{G}_{e}^{+, \sigma} \text { in }[0, S) \times \mathbf{R}
$$


holds from Lemma 7.6.

For each $\sigma \in(0,1)$ and $\xi \in \mathbf{R}$ the last inequality implies that

$$
\begin{aligned}
\bar{u}(0, \xi) & =\left(\limsup _{\rho \downarrow 0} \operatorname{su}^{\varepsilon}\right)(0, \xi) \\
& =\lim _{\rho \downarrow 0} \sup \left\{u^{e}(t, x) ; 0 \leq t<\rho,|x-\xi|<\rho, 0<\varepsilon<\rho\right\} \\
& \leq \lim _{\rho \downarrow 0} \sup \left\{\hat{G}_{\varepsilon}^{+, \sigma}(t, x ; \xi) ; 0 \leq t<\rho,|x-\xi|<\rho, 0<\varepsilon<\rho\right\} .
\end{aligned}
$$

Since Lemma 5.1(i) yields $G_{e}^{\sigma} \rightarrow G_{0}^{\sigma}$ as $\varepsilon \rightarrow 0$ locally uniformly in $\mathbf{R}$ and $G_{0}^{\sigma}(0)=0$, we observe that

$$
\lim _{\rho \downarrow 0} \sup \left\{G_{\varepsilon}^{\sigma}(t, x-\xi) ; 0 \leq t<\rho,|x-\xi|<\rho, 0<\varepsilon<\rho\right\}=0
$$

Since $u_{0}^{e}$ converges to $u_{0}$ uniformly in $\mathbf{R}$ this now yields

$$
\begin{array}{rlrl}
\bar{u}(0, \xi) & \leq \lim _{\rho \downarrow 0} \sup \left\{\hat{G}_{e}^{+, \sigma}(t, x ; \xi) ; 0 \leq t<\rho,|x-\xi|<\rho, 0<\varepsilon<\rho\right\} \\
& =u_{0}(\xi)+\sigma \quad & \text { for } \sigma \in(0,1) \text { and } \xi \in \mathbf{R} .
\end{array}
$$

Since $\sigma \in(0,1)$ is arbitrary we thus obtain

$$
\bar{u}(0, \xi) \leq u_{0}(\xi) \text { for } \xi \in \mathbf{R}
$$

Similar argument implies that

$$
\underline{u}(0, \xi) \geq u_{0}(\xi) \text { for } \xi \in \mathbf{R} .
$$

Proof of Theorem 2.11. Let $\bar{u}$ and $\underline{u}$ be same as in Lemma 7.7. We may assume that

$$
-\infty<\underline{u} \leq \bar{u}<\infty \quad \text { in }[0, T) \times \mathbf{R} .
$$

Indeed, for each $S \in(0, T)$ and $\sigma \in(0,1)$, Lemma 7.6 yields

$$
u^{e}(t, \boldsymbol{x}) \leq \hat{G}_{e}^{+, \sigma}(t, \boldsymbol{x} ; \boldsymbol{x}) \leq B^{\sigma} T+\max u_{0}^{e}+\sigma, \quad(t, \boldsymbol{x}) \in[0, S) \times \mathbf{R},
$$

so that $\bar{u} \leq B^{\sigma} T+\max u_{0}+\sigma<\infty$ on $[0, S) \times \mathbf{R}$. The proof for $\underline{u}>-\infty$ is similar. 
Then $\bar{u}$ (resp. $\underline{u}$ ) is a subsolution (resp. supersolution) of $\left(E_{0}\right)$ in $(0, T) \times \mathbf{R}$ by General Stability theorem 2.9. Since Lemma 7.7 yields that $\bar{u}(0, x) \leq$ $u_{0}(x) \leq \bar{u}(0, x)$ for $x \in \mathbf{R}$, Comparison theorem 2.7 implies that

$$
\bar{u} \leq \underline{u} \quad \text { in } \quad[0, T) \times \mathbf{R}
$$

so that

$$
u:=\bar{u}=\underline{u} \text { in }[0, T) \times \mathbf{R} .
$$

Thus we obtain the generalized solution $u \in C([0, T) \times \mathbf{R})$ of $\left(E_{0}\right)$ in $(0, T) \times \mathbf{R}$ with initial data $u_{0}$ and local uniform convergence $u^{\varepsilon} \rightarrow u$ in $[0, T) \times \mathbf{R}$.

\section{$\S$ Appendix.}

We compare various convergence for convex functions and monotone functions on $\mathbf{R}$ for the reader's convenience. For more general results on convex functions defined in a Hilbert space the reader is referred to a book of Attouch [A].

Let $B$ be a maximal monotone operator from $\mathbf{R}$ to $\mathbf{R}$. In other words it is defined in an interval $I$ and its value $B(p)$ for $p \in I$ is a closed nonempty set in $\mathbf{R}$ that satisfies

(i) (monotonicity) $\left(q_{1}-q_{2}\right)\left(p_{1}-p_{2}\right) \geq 0$ for $q_{i} \in B\left(p_{i}\right)$;

(ii)(maximality) For $p \in I, B(p)$ is the closure of the interval

$$
\left(\sup _{r<p}(\sup B(r)), \inf _{r>p}(\inf B(r))\right)
$$

with convention that inf $B(r)=+\infty, \sup B(r)=-\infty$, if $B(r)$ is empty. The set $I$ is called the domain (of definition of) $B$. Because of monotonicity $B(p)$ is a singleton except (at most) countably many $p$ 's. The set $P$ of $p \in I$ at which $B(p)$ is not a singleton is called the discontinuity of $B$. If $B(p)$ is a singleton, its element is denoted $b(p)$. Let $b^{*}$ and $b_{*}$ denote the upper and lower semicontinuous envelope of a single valued function $b$ defined on $\mathbf{R} \backslash P$, respectively, with convention that $b(p)=+\infty$ for $p>\sup I, b(p)=-\infty$ for $p<\inf I ; b$ is called the nondecreasing function associated with the maximal monotone operator $B$. By maximality $B(p)$ for $p \in P$ is the closure of $\left(b_{*}(p), b^{*}(p)\right)$. 
Lemma A.1. Let $B$ and $B_{\varepsilon}(\varepsilon>0)$ be maximal monotone operators form $\mathbf{R}$ to $\mathbf{R}$. Let $b$ and $b_{\varepsilon}$ be the nondecreasing functions associated with $B$ and $B_{\varepsilon}$, respectively. Let $I$ and $P$ denote the domain and the discontinuity of $B$, respectively. The following conditions are equivalent.

(i) (pointwise convergence) $\lim _{\varepsilon \rightarrow 0}\left(b_{\varepsilon}\right)^{*}(p)=b(p)=\lim _{\varepsilon \rightarrow 0}\left(b_{\varepsilon}\right)_{*}(p)$ for all $p \in \mathbf{R} \backslash P$.

(ii) Limsup $\operatorname{lio}_{\varepsilon} B_{\varepsilon}(p) \subset B(p)$ for all $p \in \mathbf{R}$ as sets in $\mathbf{R}$, where

$$
\operatorname{Limsup}_{\varepsilon \rightarrow 0} Z_{\varepsilon}=\cap_{\varepsilon>0} \overline{\cup_{0<\delta<\varepsilon} Z_{\delta}}
$$

for a family of sets $\left\{Z_{\varepsilon}\right\}_{e}>0$. In other words for any $q_{\varepsilon} \in B_{\varepsilon}(p)$ converging to $q_{0} \in \mathbf{R}$ we have $q_{0} \in B(p)$.

(iii) (uppersemiconvergence of graphs) Limsup $p_{\varepsilon \rightarrow 0}\left(\right.$ graph $\left.B_{\varepsilon}\right) \subset$ graph $B$, i.e. for any sequence $\left(p_{\varepsilon}, q_{\varepsilon}\right)$ with $q_{\varepsilon} \in B_{\varepsilon}\left(p_{\varepsilon}\right)$ converging to $\left(p_{0}, q_{0}\right) \in \mathbf{R}^{2}$ we have $q_{0} \in B\left(p_{0}\right)$ with $p_{0} \in I$. Here graph $A$ denotes the graph of a set-valued function $A$, i.e. graph $A=\{(p, q) ; q \in A(p)$ and $p \in I\}$.

(iv) (uniform convergence) For each compact set $K$ in $I \backslash P,\left(b_{\varepsilon}\right)^{*}$ and $\left(b_{\varepsilon}\right)_{*}$ converges to $b$ uniformly in $K$ as $\varepsilon \rightarrow 0$. Moreover, $\lim _{\varepsilon \rightarrow 0}\left(b_{\varepsilon}\right)^{*}(p)=$ $b(p)=\lim _{\varepsilon \rightarrow 0}\left(b_{e}\right)_{*}(p)$ for $p \in \mathbf{R} \backslash I$.

Proof. (i) $\Rightarrow$ (ii). For $p \in I \backslash P$ the convergence (i) implies Limsup $B_{\varepsilon}(p) \subset$ $B(p)$. Since $P$ is a countable set, for $p \in P$ there is $p_{l} \rightarrow p(l \rightarrow \infty)$ with $p_{l} \notin P$ and $p_{l}>p$. By monotonicity we have

$$
\sup B_{\varepsilon}(p) \leq\left(b_{\varepsilon}\right)^{*}\left(p_{l}\right)
$$

Sending $\varepsilon \rightarrow 0$ yields

$$
\limsup _{\varepsilon \rightarrow 0}\left\{\sup B_{\varepsilon}(p)\right\} \leq b\left(p_{l}\right)
$$

since $\left(b_{\varepsilon}\right)^{*}\left(p_{l}\right) \rightarrow b\left(p_{l}\right)$ by (i). Letting $l \rightarrow \infty$ yields

$$
\limsup _{\varepsilon \rightarrow 0}\left\{\sup B_{\varepsilon}(p)\right\} \leq b^{*}(p+0)=b^{*}(p) .
$$

Similarly we have

$$
\liminf _{\varepsilon \rightarrow 0}\left\{\inf B_{\varepsilon}(p)\right\} \geq b_{*}(p)
$$

so the convergence (ii) follows for $p \in P$. 
(ii) $\Rightarrow$ (iii). We may assume that $p_{\varepsilon}>p_{0}$ for sufficiently small $\varepsilon>0$ or $p_{\varepsilon}<p_{0}$ for sufficiently small $\varepsilon>0$ by taking a subsequence, since (ii) implies $q_{0} \in B\left(p_{0}\right)$ if $p_{\varepsilon}=p_{0}$ for small $\varepsilon>0$. We may also assume the convergence $p_{\varepsilon} \rightarrow p_{0}$ is monotone. If $p_{\varepsilon} \downarrow p_{0}$ then by monotonicity of $B_{\varepsilon}$ we see that

$$
\left(b_{\varepsilon}\right)_{*}\left(p_{0}\right) \leq\left(b_{\varepsilon}\right)_{*}\left(p_{\varepsilon}\right) \leq q_{\varepsilon} \leq\left(b_{\varepsilon}\right)^{*}\left(p_{\varepsilon_{0}}\right) \quad \text { for } \varepsilon \in\left(0, \varepsilon_{0}\right) .
$$

Sending $\varepsilon$ to zero yields

$$
\begin{aligned}
b_{*}\left(p_{0}\right) & \leq \liminf _{\varepsilon \rightarrow 0}\left(b_{\varepsilon}\right)_{*}\left(p_{0}\right) \leq \liminf _{\varepsilon \rightarrow 0}\left(b_{\varepsilon}\right)_{*}\left(p_{\varepsilon}\right) \\
& \leq q_{0} \leq \limsup _{\varepsilon \rightarrow 0}\left(b_{\varepsilon}\right)^{*}\left(p_{\varepsilon_{0}}\right) \leq b^{*}\left(p_{\varepsilon_{0}}\right)
\end{aligned}
$$

by (ii) at $p=p_{0}$ and $p=p_{\varepsilon_{0}}$. Sending $\varepsilon_{0} \rightarrow 0$ now yields

$$
b_{*}\left(p_{0}\right) \leq q_{0} \leq b^{*}\left(p_{0}\right)
$$

We thus observe that $q_{0} \in B\left(p_{0}\right)$. Note that $p_{0} \in I$ since otherwise $b_{*}\left(p_{0}\right)=$ $b^{*}\left(p_{0}\right)$ equals either $+\infty$ or $-\infty$ contradicting the finiteness of $q_{0}$. If $p_{\varepsilon} \uparrow p_{0}$ the similar proof yields $q_{0} \in B\left(p_{0}\right)$ with $p_{0} \in I$.

(iii) $\Rightarrow$ (iv). Since the uppersemiconvergence of graph is equivalent to the statement that for each $\eta>0$ and a bounded set $Z$ in $\mathbf{R}^{2}$ there exists $\varepsilon_{0}>0$ that satisfies

$$
\text { ( graph } \left.B_{\varepsilon}\right) \cap Z \subset\{(p, q) \in Z ; d((p, q) \text {, graph } B)<\eta\}
$$

for $\varepsilon<\varepsilon_{0}$, the uniform convergence of $\left(b_{\varepsilon}\right)^{*}$ and $\left(b_{\varepsilon}\right)_{*}$ in $K$ follows easily. The last assertion is also easy to prove.

(iv) $\Rightarrow$ (i). This is trivial so the proof of Lemma A.1 is now complete.

Remark A.2. The condition (i) - (iv) implies

$$
\operatorname{Liminf}_{\varepsilon \rightarrow 0}\left(\text { graph } B_{\varepsilon}\right) \supset \operatorname{graph} B
$$

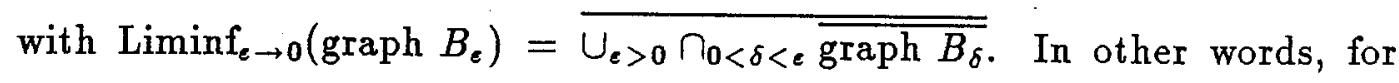
each $q \in B(p)$ there is a sequence $\left(p_{\varepsilon}, q_{\varepsilon}\right)$ with $q_{e} \in B\left(p_{\varepsilon}\right)$ that converges to $(p, q)$ as $\varepsilon \rightarrow 0$. So the condition (i) -(iv) implies the graph convergence of $B_{e}$ to $B$ as explained in [A].

We now discuss the relation of convergence of convex functions and its subdifferentials. For simplicity we consider real-valued convex functions defined everywhere on $\mathbf{R}$. 
Lemma A.3. Let $W$ and $W_{\varepsilon}(\varepsilon>0)$ be (real-valued) convex functions on $\mathbf{R}$ (so that they are continuous on $\mathbf{R}$ ). If $W_{\varepsilon} \rightarrow W$ pointwise as $\varepsilon \rightarrow 0$, then

$$
\operatorname{Limsup}_{\varepsilon \rightarrow 0}\left(\partial W_{\varepsilon}(p)\right) \subset \partial W(p) \quad \text { for } p \in \mathbf{R}
$$

where $\partial W$ denotes the subdifferential of $W$. Moreover, for each $p_{1} \leq p_{2}$ the set $\cup_{p_{1} \leq p \leq p_{2}} \partial W_{\varepsilon}(p)$ is contained in a bounded set in $\mathbf{R}$ independent of small $\varepsilon>0$. In particular, $W_{\varepsilon}$ converges to $W$ locally uniformly in $\mathbf{R}$.

Proof. By definition $q_{\varepsilon} \in \partial W_{\varepsilon}(p)$ means

$$
W_{\varepsilon}(p+h)-W_{\varepsilon}(p) \geq q_{\varepsilon} \cdot h \quad \text { for all } h \in \mathbf{R} \text {. }
$$

Since $W_{\varepsilon}$ converges to $W$ pointwise, sending $\varepsilon \rightarrow 0$ yields

$$
W(p+h)-W(p) \geq q_{0} \cdot h, \quad q_{0} \in \partial W(p)
$$

if $q_{e} \rightarrow q_{0}$. Thus $q_{0} \in \partial W(p)$.

If $q_{e}$ tends to $+\infty$ by taking a subsequence, then sending $\varepsilon$ to zero in (A.1) we observe for any $m>0$

$$
W(p+h)-W(p) \geq m \cdot h \quad \text { for all } h>0 \text {. }
$$

However, this contradicts finiteness of $W(r)$ for $r>p$. Similarly, there is no subsequence such that $q_{e} \rightarrow-\infty$. In particular $\partial W(p)$ is bounded.

Thus $\partial W_{\varepsilon}(p)$ belongs to a bounded set $Z_{p} \subset \mathbf{R}$ (containing $\partial W(p)$ ) for small $\varepsilon>0$. Since $\partial W_{e}$ is maximal monotone,

$$
\cup_{p_{1} \leq p \leq p_{2}} \partial W_{\varepsilon}(p) \subset\left[\inf Z_{p_{1}}, \sup Z_{p_{2}}\right] \quad \text { for small } \varepsilon>0
$$

This implies that $W_{e}$ is Lipschitz on $\left[p_{1}, p_{2}\right]$ with a Lipschitz constant independent of $\varepsilon$. Since $W_{\varepsilon}$ converges to $W$ pointwise, the uniform Lipschitz continuity implies boundedness of $\left\{W_{\varepsilon}\right\}$ on $\left[p_{1}, p_{2}\right]$. From Ascoli-Arzela's theorem subsequence of $\left\{W_{\varepsilon}\right\}$ converges to some function uniformly in $\left[p_{1}, p_{2}\right]$. Since $W_{e}$ converges to $W$ pointwise, $W_{e}$ converges to $W$ uniformly in $\left[p_{1}, p_{2}\right]$. Since $p_{1}$ and $p_{2}$ are arbitrary, the proof is now complete. 
Lemma A.4. Let $W_{\varepsilon}$ and $W(\varepsilon>0)$ be real-valued convex functions on $\mathbf{R}$. Assume that $W_{\varepsilon}$ converges to $W$ pointwise as $\varepsilon \rightarrow 0$. Then the distributional second derivatives $W_{e}^{\prime \prime}$ converges to $W^{\prime \prime}$ weakly as measures.

Proof. By Lemma A.3, $\left\{\left(W_{\varepsilon}^{\prime}\right)^{*}\right\}$ is locally bounded for small $\varepsilon>0$. Thus $\left\{W_{\varepsilon}^{\prime \prime}\right\}$ is locally bounded as measures. Since. $W_{\varepsilon} \rightarrow W$ locally uniformly $W_{\varepsilon}^{\prime \prime} \rightarrow W^{\prime \prime}$ in distribution sense. Since $\left\{W_{\varepsilon}^{\prime \prime}\right\}$ is bounded as measures on every bounded interval, $W_{\varepsilon}^{\prime \prime}$ converges to some measure $\mu$ weakly as measures [Giu, Appendix], i.e.

$$
\int_{\mathbf{R}} \varphi W_{\varepsilon}^{\prime \prime} \rightarrow \int_{\mathbf{R}} \varphi \mu, \quad \varphi \in C_{0}^{0}(\mathbf{R})
$$

by taking a subsequence. Since $W_{e}^{\prime \prime} \rightarrow W^{\prime \prime}$ in distribution sense, $\mu=W^{\prime \prime}$ so the proof is now complete.

Lemma A.5. Let $\stackrel{\circ}{w}$ and $\stackrel{\circ}{w}^{\varepsilon}(\varepsilon>0)$ be unit Wulff functions of $\alpha \not \equiv 0$ and $\alpha_{\varepsilon} \not \equiv 0(\in \mathcal{A})$. Assume that $\alpha_{\varepsilon}$ converges to $\alpha$ weakly as measures as $\varepsilon \rightarrow 0$.

(i) $\stackrel{\circ}{w}^{\varepsilon}$ converges to $\stackrel{\circ}{w}$ locally uniform in $\left(\beta_{-}, \beta_{+}\right)$, where $\left(\beta_{-}, \beta_{+}\right)$is defined for $\stackrel{\circ}{w}$ as in Definition 4.1 .

(ii) $\partial \dot{0}^{e}$ converges to $\partial \dot{w}$ in the sense of uppersemiconvergence of graphs as in Lemma A.1(iii), where $\partial \dot{w}^{\circ}$ is interpreted as in Lemma 5.1.

(iii) (i) and (ii) are still valid for unit cutoff Wulf functions $\tilde{\tilde{w}}$ with slopes $\left(q_{-}, q_{+}\right), q_{-}<0<q_{+}$, independent of $\varepsilon$ even if $\alpha_{e} \equiv$ for some $\varepsilon \geq 0$.

Proof. Similarly to define $\beta$ from $\alpha$ in Definition 4.1, we set

$$
\beta_{\varepsilon}(p)=\int_{+0}^{p} \alpha_{\varepsilon}(q) d q+ \begin{cases}0 & \text { if } 0 \notin P_{\varepsilon} \\ \Delta_{\varepsilon}(0) / 2 & \text { if } 0 \in P_{\varepsilon}\end{cases}
$$

where $P_{\varepsilon}$ is the singularity of $W_{\varepsilon}$ with $W_{\varepsilon}^{\prime \prime}=\alpha_{\varepsilon}$. Since $\alpha_{\varepsilon} \rightarrow \alpha$ weakly as measures, it is standard to conclude $\beta_{\epsilon} \rightarrow \beta$ pointwise except at the singularity $P$ of $W$ with $W^{\prime \prime}=\alpha$. Lemma A.1(ii) implies that $\partial W_{\varepsilon} \rightarrow \partial W$ in the sense of uppersemiconvergence of graphs. Thus its inverse $\left(\partial W_{\varepsilon}\right)^{-1}$ converges to $(\partial W)^{-1}$ in the sense of uppersemiconvergence of graphs. Since $\partial \stackrel{\circ}{w}^{e}=\left(\partial W_{\varepsilon}\right)^{-1}, \partial \stackrel{\circ}{w}=(\partial W)^{-1}$ by definition, this yields (ii). Since the uniform convergence of $\left(\stackrel{\circ}{w}^{e}\right)^{\prime}$ outside singularity of $(\stackrel{\circ}{w})^{\prime}$ is obtained by Lemma A.1(iv), $\stackrel{0}{w^{e}}$ converges to $\stackrel{\circ}{w}$ locally uniformly in $\left(\beta_{-}, \beta_{+}\right)$. The proof for cutoff Wulff functions is similar even if $\alpha_{\varepsilon} \equiv 0$ for some $\varepsilon \geq 0$ so is omitted. 
Proof of Lemma 5.1. Part (i) and (ii) follow from (i) and (ii) of Lemma A.5, respectively. Part (iii) follows from Lemma A.1(iv) and Lemma A.5 (i). Part (iv) follows from (i),(ii),(iii) and definition of cutoff Wulff functions.

Remark A.6. It is well known [A] that epigraph convergence of convex functions in $\mathbf{R}^{n}$ is equivalent to the graph convergence of subdifferentials. So it is equivalent to epigraph convergence of convex conjugate functions since convergence of subdifferentials and its inverses as graphs is the same. Such conditions is extended for convex functions defined in a Hilbert space by replacing epigraph convergence by Mosco convergence. Note that for a function defined in $\mathbf{R}^{n}$ the uniform convergence implies epigraph convergence.

\section{REFERENCES}

[AG] S. B. Angenent and M. E. Gurtin, Multiphase thermomechanics with interfacial structure 2. Evolution of an isothermal interface, Arch. Rational Mech. Anal. 108 (1989), 323-391.

[A] H. Attouch, Variational Convergence for Functions and Operators, Pitman, Boston, 1984.

[Ba] G. Barles, Solutions de viscosité des équations de Hamilton-Jacobi, Springer-Verlag, Paris, 1994.

[BaP1] G. Barles and B. Perthame, Discontinuous solutions of deterministic optimal stopping problems, Math. Model. Numer. Anal. 21 (1987), 557-579.

[BaP2] G. Barles and B. Perthame, Exit time problems in control and vanishing viscosity solutions of Hamilton-Jacobi-Bellman equations, SIAM J. Control Optim. 26 (1989), 1113-1148.

[BP] H.Brezis and A. Pazy, Convergence and approximation of semigroups of nonlinear operators in Banach spaces, J. Funct. Anal. 9 (1972), 63-74.

[CGS] Y.-G. Chen, Y. Giga and K. Sato, On instant extinction for very fast diffusion equations, Discrete and Continuous Dynamic Systems, to appear.

[Ch] A. A. Chernov, Modern Crystallography III, Crystal Growth, Solid-State Sciences 36, Springer Verlag 1984, (especially §3.3).

[CIL] M. G. Crandall, H. Ishii and P. L. Lions, User's guide to viscosity solutions of second order partial differential equations, Bull. Amer. Math. Soc. 27 (1992), 1-67.

[E] L. C. Evans, The perturbed test function method for viscosity solutions of nonlinear PDE, Proc. Royal Soc. of Edinburgh 111A (1989), 359-375. 
[El] C. M. Elliott, Approximation of curvature dependent interface motion (1996), preprint.

[FP] A.Fasano and M.Primicero, Liquid flow in partially saturated porous media, J. Inst. Maths. Applics. 23 (1979), 503-517.

[FG] T. Fukui and Y. Giga, Motion of a graph by nonsmooth weighted curvature, In: World Congress of Nonlinear Analysts '92 (ed. V. Lakshmikantham), Walter de Gruyter, Berlin, vol. I (1996), pp.4756.

[GG1] M.-H. Giga and Y. Giga, Geometric evolution by nonsmooth interfacial energy, In: Nonlinear Analysis and Applications (eds. N. Kenmochi, A. Damlamian, M. Niezgódka and A. Visintin) Proceedings of Banach Center Minisemester, November-December, 1994, Gakuto International Series Mathematical Sciences and Applications, 7 (1995) pp.125-140, Gakuto, Tokyo.

[GG2] M.-H. Giga and Y. Giga, Consistency in evolutions by crystalline curvature, Proc. Free Boundary Problems '95, Theory and Applications (ed. M. Niezgódka), Zakopane, Poland(1995), to appear.

[GG3] M.-H. Giga and Y. Giga, Evolving graphs by singular weighted curvature, Arch. Rational. Mech. Anal., to appear.

[GG4] M.-H. Giga and Y. Giga, A subdifferential interpretation of crystalline motion under nonuniform driving force, Hokkaido University Preprint Series in Mathematics \#344 (1996).

[GGu] Y. Giga and M. E. Gurtin, A comparison theorem for crystalline evolution in the plane, Quart. J. Appl. Math., in press.

[Gir] P. M. Girão, Convergence of a crystalline algorithm for the motion of a simple closed convex by weighted curvature, SIAM J. Numer. Anal. 32 (1995), 886-899.

[GirK1] P. M. Girão and R. V. Kohn, Convergence of a crystalline algorithm for the heat equation in one dimension and for the motion of a graph by weighted curvature, Numer. Math. 67 (1994), 41-70.

[GirK2] P. M. Girão and R. V. Kohn, The crystalline algorithm for computing motion by curvature, In: Variational Methods for Discontinuous Structure (eds. R. Serapioni and F. Tomarelli) Birkhauser, to appear.

[Giu] E. Giusti, Minimal Surfaces and Functions of Bounded Variations, Birkhauser, Boston, 1984.

[Gu] M. E. Gurtin, Thermomechanics of Evolving Phase Boundaries in the Plane, Oxford, Clarendon Press, 1993.

[I] H.Ishii, A boundary value problem of the Dirichlet type for HamiltonJacobi equations, Ann. Scuola. Norm. Pisa Cl. Sci. (4) 16 (1989), 105-135.

[ISo] K. Ishii and M.-H. Soner, Regularity and convergence of crystalline 
motion, preprint (1996).

[LSU] O. A. Ladyzhenskaya, V. Solonnikov and N. Ural'ceva, Linear and Quasilinear Equations of Parabolic Type, Translations of Mathematical Monographs, Amer. Math. Soc. 23 (1968).

[Rob] S. Robert, A line element algorithm for curve flow problems in the plane, J. Austral. Math. Soc. Ser. B 35 (1993), 244-261.

[R] A. R. Roosen, Crystalline curvature and flat flow in a diffusion field, preprint (1994).

[S] H. Spohn, Surface dynamics below the roughening transition, J. Phys. I. France 3 (1993), 69-81.

[T1] J. Taylor, Constructions and conjectures in crystalline nondifferential geometry, In: Differential Geometry (eds. B. Lawson and K. Tanenblat), Proceedings of the Conference on Differential Geometry, Rio de Janeiro, Pitman Monographs in Pure and Applied Math. 52 (1991) pp.321-336, Pitman, London.

[T2] J. Taylor, Mean curvature and weighted mean curvature, Acta Metall. 40 (1992), 1475-1485.

[T3] J. Taylor, Motion of curves by crystalline curvature, including triple junctions and boundary points, In: Differential Geometry: Partial Differential Equations on Manifolds (eds. R. Greene and S. T. Yau), Proc. Symp. Pure Math., 54(1993) Part I, pp. 417-438, Amer. Math. Society Providence, RI.

[TCH] J. Taylor, J. Cahn and C. Handwerker, Geometric models of crystal growth, Acta Metall. 40 (1992), 1443-1474. 